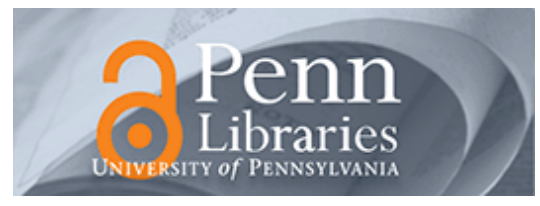

University of Pennsylvania ScholarlyCommons

April 1971

\title{
Access Control and Retrieval Optimization Functions of the Supervisor for an Extended Data Management Facility
}

Judith Irene Hirsch

University of Pennsylvania

Follow this and additional works at: https://repository.upenn.edu/cis_reports

\section{Recommended Citation}

Judith Irene Hirsch, "Access Control and Retrieval Optimization Functions of the Supervisor for an Extended Data Management Facility", . April 1971.

University of Pennsylvania Department of Computer and Information Science Technical Report No. MS-CIS-71-21.

This paper is posted at ScholarlyCommons. https://repository.upenn.edu/cis_reports/825

For more information, please contact repository@pobox.upenn.edu. 


\title{
Access Control and Retrieval Optimization Functions of the Supervisor for an Extended Data Management Facility
}

\author{
Abstract \\ The purpose of the Supervisor in an Extended Data Management Facility (EDMF) is to direct the Facility's \\ handling of a user's request for service. The Supervisor fulfills its task through the use of five main \\ functions: Access Control, Retrieval Initialization, File Searching, Record Validating and Record \\ Formatting. The major and most important component of the Retrieval Initialization phase is the Retrieval \\ Optimization subfunction. This report is concerned mainly with the design and implementation of the \\ Access Control and Retrieval Optimization functions. Macro instructions are the mechanism through \\ which a user's program can call upon the ECMF. The Authority Item check is the EDMF's security control \\ over file access while the Prime Keyword Search is the method used to optimize the retrieval strategy. The \\ Authority Item check and the Prime Keyword Search are two of the major concepts of the Extended Data \\ Management Facility.
}

\section{Comments}

University of Pennsylvania Department of Computer and Information Science Technical Report No. MSCIS-71-21. 
University of Pennsylvania

THE MOORE SCHOOL OF EIECTRICAL ENGINEERING

Philadelphia, Pennsylvania

\begin{abstract}
TECHNICAL REPORT
ACCESS CONTROL AND RETRIEVAL OPTIMIZATION FUNCTIONS OF THE SUPERVISOR

FOR AN EXTENDED DATA MANAGEMENT FACILITY
\end{abstract}

by

Judith Irene Hirsch

April 1971

\begin{abstract}
Submitted to the
Office of Naval Research

Information Systems Branch

Arlington, Virginia

under

Contract NO0014-67-A-0216-0014

Research Project NR 049-153
\end{abstract}

Reproduction in whole or in part is permitted for any purpose of the

United States Government

Moore School Report No. 71-21 
ACCESS CONTROL AND RETRIEVAL OPTIMIZATION

FUNCTIONS OF THE SUPERVISOR

FOR AN EXTENDED DATA MANAGEMENT FACIIITY

\section{Abstract}

The purpose of the Supervisor in an Extended Data Management Facility (EDMF) is to direct the Facility's handling of a user's request for service. The Supervisor fulfills its task through the use of five main functions: Access Control, Retrieval Initialization, File Searching, Record Validating and Record Formatting. The major and most important component of the Retrieval Initialization phase is the Retrieval Optimization subfunction. This report is concerned mainly with the design and implementation of the Access Control and Retrieval Optimization functions. Macro instructions are the mechanism through which a user's program can call upon the EDMF. The Authority Item check is the EDMF's security control over file access while the Prime Keyword Search is the method used to optimize the retrieval strategy. The Authority Item check and the Prime Keyword Search are two of the major concepts of the Extended Data Management Facility. 
DOCUMENT CONTROL DATA - R \& D

(Sccurity clessification of tillo, body of abstract and indexing annotation nust be entered when the oversll report is clasultled) 1. ORIGINATING ACTIVITY (Corporate author)

The Moore School of Electrical Engineering

University of Pennsylvania

2a. REPORT SECURITY CLASSIFICATION

Phila., Pa. 19104

3. REPORT TITLE

ACCESS CONTROL AND RETRIEVAL OPTIMIZATION FUNCTIONS OF THE SUPERVISOR

FOR AN EXTENDED DATA MANAGEMENT FACIIITY

I DESCRIPTIVE NOTES (Type of report and, inclusive dates)

Technical Report

8. AUTHORISI (FIrst name, middlO initial, last name)

Judith I. Hirsch

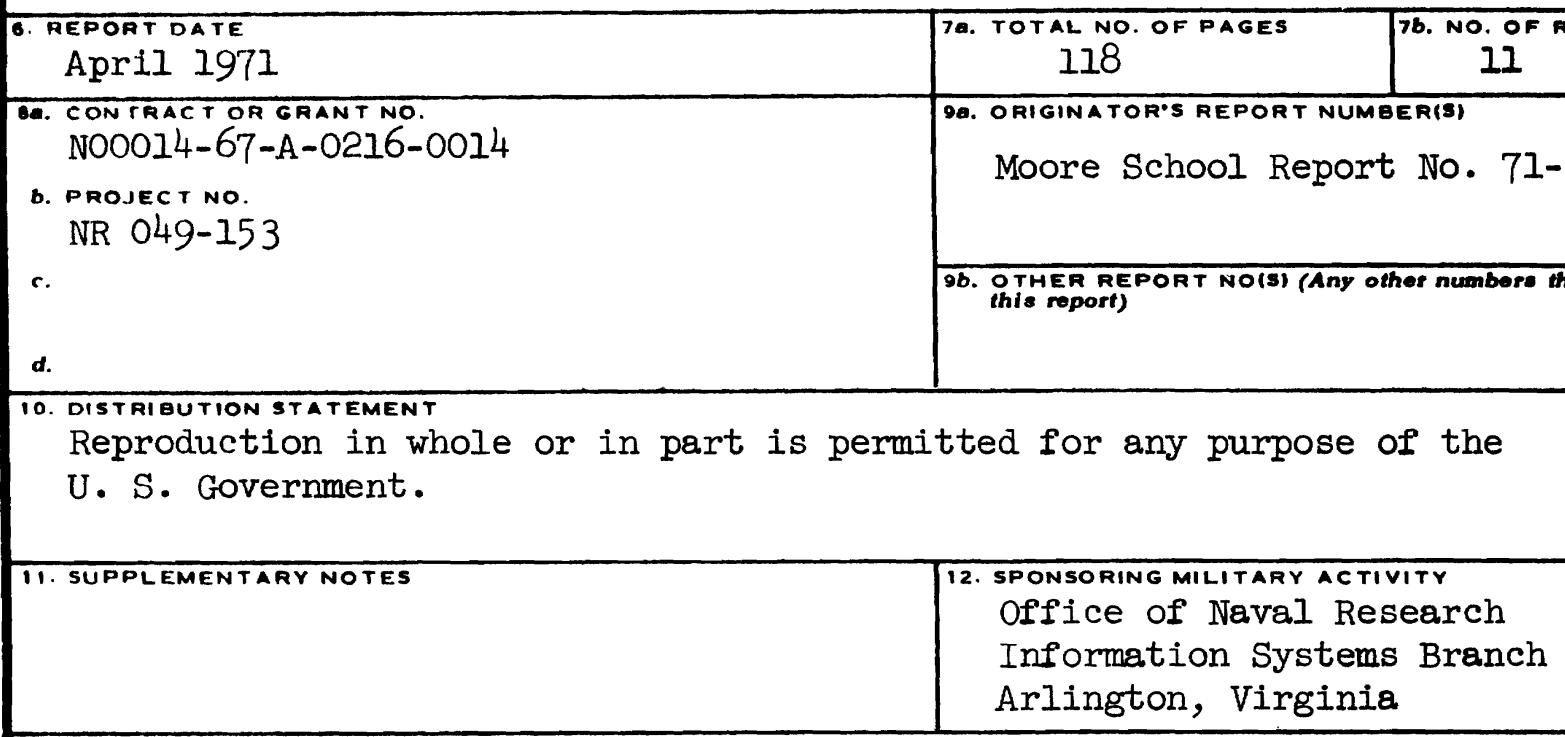

13. ABSTRACT

The purpose of the Supervisor in an Extended Data Management Facility (EDMF) is to direct the Facility's handling of a user's request for service. The Supervisor fulfills its task through the use of five main functions: Access Control, Retrieval Initialization, File Searching, Record Validating and Record Formatting. The major and most important component of the Retrieval Initialization phase is the Retrieval Optimization subfunction. This report is concerned mainly with the design and implementation of the Access Control and Retrieval Optimization functions. Macro instructions are the mechanism through which a user's program can call upon the EDMF. The Authority Item check is the EDMF's security control over file access while the Prime Keyword Search is the method used to optimize the retrieval strategy. The Authority Item check and the Prime Keyword Search are two of the major concepts of the Extended Data Management Facility 
Security Classification

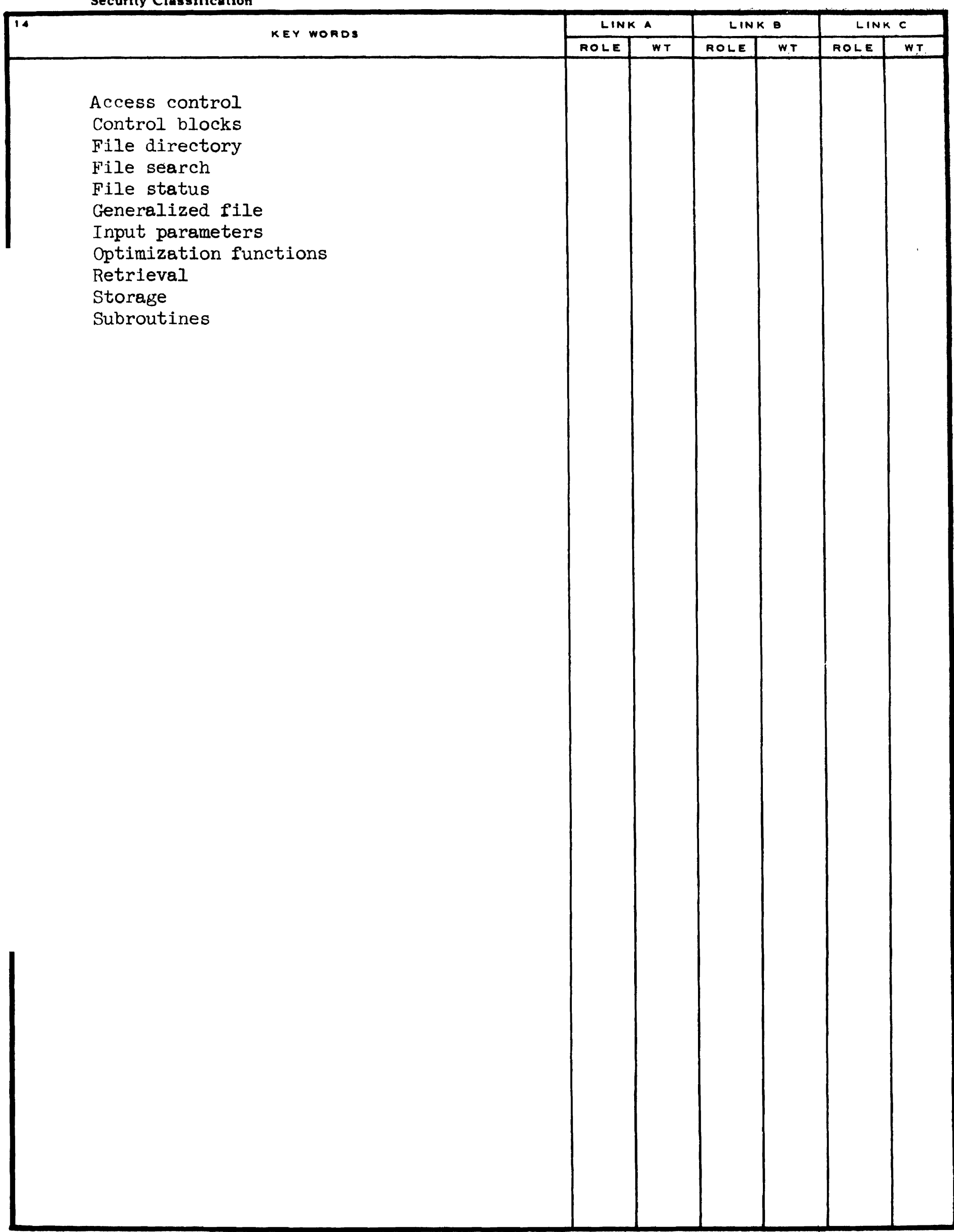


TABIE OF CONTENTS

\section{Page}

CHAPTER I INTRODUCTION 1

1.I The Extended Data Management Facility I

1.2 The Supervisor of the EDMF 2

1.3 The Scope of the Thesis 2

CHAPTER 2 THE OPEN FUNCTION

2.1 Definitions $\quad \cdot \quad 4$

2.1.1 Attribute-Value Pair 4

2.1 .2 Record 4

2.1 .3 Keywords 6

$\begin{array}{lll}2.1 .4 & \text { Keyword Lists } & 7\end{array}$

2.1.5 File and Directory 9

2.1.6 Generalized File Structure 11

2.1.7 Request Description 13

2.1.8 Entering the EDMF 14

2.2 Purpose of the Open Function 16

2.3 Access Control 16

2.3.1 Introduction $\quad 16$

2.3.2 File Level Check 17

2.3.3 Partitioning the File 18

2.3.4 User's Authority Item 20

2.4 Control Blocks 21

2.4.1 Service Status Block 22

2.4.2 File Status Block 23

2.5 Return to User _ _ . 24 
TABLE OF CONTENTS (continued)

\section{Page}

2.6 The EDMF's OPN Macro

25

CHAPTER 3 THE RETRIEVAL INITTALIZATION FUNCTION 26

3.1 Purpose 26

3.2 Control Blocks 26

3.2.1 DMS Open $\quad 26$

$\begin{array}{lll}3.3 & \text { Retrieval Optimization } & 27\end{array}$

3.3.1 Prime Keywords 28

3.4 ISAM Keys 29

3.5 Record Format Numbers 30

3.6 Control Passed to the File Searching Function 31

3.7 The EDMF's RETR Macro . 31

CHAPTER 4 THE CLOSE FUNCTION 32

4.1 Purpose 32

4.2 Control Blocks 32

4.3 Return to User 33

4.4 The EDMF's CLSE Macro 33

$\begin{array}{lll}\text { CHAPTER } 5 & 34\end{array}$

$\begin{array}{ll}\text { BIBLIOGRAPHY } & 35\end{array}$

APPENDIX A MACROS A-I.

A.I Open Macro A-1

A.1.1 Generated Parameter List A-2

A.2 Retrieval Macro - A-4 
TABLE OF CONTENTS (continued)

A.2.1 Generated Parameter Iist

Page

A.3 Close Macro

A-6

A-7

A.3.1 Generated Parameter Iist

A-8

APPENDIX B ROUTINES

B-1

B.I Routine OPNPROC

B-1

B.I.1 Entry Points

B-I

B.1.2 Exit Points

B-1

B.1.3 External Subroutine Calls

B-1

B.1.4 Input Parameter List

B-3

B.1.5 Register Conventions

B-3

B.1.6 Internal Work Area

B- 4

B.1.7 Internal Codes

B-6

B.1.8 Return Codes

B- 8

B.1.9 Flowchart

B-8

B.2 Routine FIFDIRSI

B- 14

B.2.1 Entry Points

B- -14

B.2.2 Exit Points

B- 14

B.2.3 External subroutine Calls

B- 14

B.2.4 Input Parameter List

B- 15

B.2.5 Register Conventions

B-16

B.2.6 Internal Work Area

B-16

B.2.7 Internal Codes

B-17

B.2.8 Return Codes

B-17

B.2.9 Flowchart

B- 18 
TABLE OF CONTENTS (continued)

B. 3 Routine MACPROC

Page

B.3.1 Entry Points

B-2I

B.3.2 Exit Points

B-2I

B.3.3 External Subroutine Calls

B-21

B.3.4 Input Parameter List

B-2I

B.3.5 Register Conventions

B-22

B.3.6 Internal Work Area

B-24

B.3.7 Internal Codes

B- 24

B.3.8 Flowchart

B-25

B-26

B.4 Routine RETRTEVE

B- 30

B.4.I Entry Points

B- 30

B.4.2 Exit Points

B-30

B.4.3 External Subroutine Calls

B- 30

B.4.4 Input Parameter List

B- 30

B.4.5 Register Conventions

B- 31

B.4.6 Internal Work Area

B- 32

B.4.7 Internal Codes

B- 34

B.4.8 Flowchart and Supplementary Diagrams

B- 35

B.5 Routine FORPROG

B- 47

B.5.1 Entry Points

B- 47

B.5.2 Exit Points

B- 47

B.5.3 Input Parameter List

B- 47

B.5.4 Register Conventions

B- 48

B.5.5 Internal Work Area

B-49

B.5.6 Internal Codes

B-49 
TABLE OF CONTENTS (continued)

B.5.7 Flowchart B-49

B.6 Routine CLSEPROC B-54

B.6.1 Entry Points B-54

B.6.2 Exit Points B-54

B.6.3 External Subroutine Calls B-54

B.6.4 Input Parameter List B-55

B.6.5 Register Conventions B-56

B.6.6 Internal Work Area B-57

B.6.7 Internal Codes. B-57

B.6.8 Return Codes B-58

B.6.9 Flowchart and Supplementary Diagram B-58

APPENDIX C CONTROL BLOCKS $\quad$ C-I

C.I File Status Block C-I

C.2 Service Status Block C-2

C.2.I User Description Block C-2

C.3 Record Format Block c-3 
CHAPTER I

INTRODUCTION

Today, there is a rapid and ever increasing growth in the total volume of information. This huge volume threatens to make the information useless unless ways can be found to manage it. The purpose of the Extended Data Management Facility (EDMF) is to provide a flexible, general purpose, time-shared file management system for the orderly accumulation and dissemination of information [9].

\subsection{The Extended Data Management Facility}

The Extended Data Management Facility is an extension of the data management system that presently exists at the Moore School on RCA's Spectra 70/46 Time Sharing Operating System (TSOS). The EDMF makes use of the services offered under TSOS, especially the Data Management System's Indexed Sequential Access Method (ISAM), and it also incorporates its own routines into the operating system.

In order to encourage the use of the EDMF, it must be relatively simple to use. The EDMF simplifies for the user the problem of designating those records that he wishes to see. The user does not need to know the actual addresses of the desired records but he merely must express as a logical expression the characteristic contents of the records. The EDMF then takes on the responsibility of determining the actual record addresses and uses these addresses to retrieve the records. The heart of the Facility is the implementation of the generalized file structure and its general retrieval algorithm as suggested by Hsiao and Harary in [8]. For an overall description of the EDMF, the reader is referred to $[9]$. 


\subsection{The Supervisor of the EDMF}

The purpose of the Supervisor in the EDMF is to direct the Facillty's handling of a user's request for service. In this capacsty, the Supervisor assumes the roles of "doorman", "foreman", "administrator", and "dispatcher". It is at first as a "doorman" who accepts the service requests and initiates their request handling routines. Then as a "foreman", the Supervisor regulates the use of the primitive storage and retrieval routines [6] and system subroutines, and also optimizes the storage and retrieval strategy for a time-sharing environment. In its role as an "administrator", the Supervisor controls the user's access to files and validates the systems output of records to the user. It is also a "dispatcher" who returns the results of the service to the user.

In directing the handling of the user's requests, the supervisor performs five main functions: Access Control, Retrieval Initialization, File Searching, Record Validating, and Record Formatting. The major and most important component of the Retrieval Initialization phase is the Retrieval Optimization subfunction. The five main functions in combination with each other satisfy the above roles which the supervisor must assume.

\subsection{The Scope of the Report}

This report is concerned mainly with the Access Control and Retrieval Initialization Functions of the Supervisor. These functions fulfill the role of "doorman" and partially those of "foreman" and "administrator". Macro instructions are the "doorman's" entrance into the request handling routines. The Prime Keyword search is the "foreman's" method of optimizing the retrieval strategy and the check of the user's Authority Item is the "administrator's" security control over file access. 
A discussion of the macro instructions and the user's Authority Item can be found in Chapter Two, the Open Function; while Chapter Three, The Retrieval Initialization Function, contains a discussion of the retrieval strategy. 
CHAPTEER 2

THE OPEN FUNCTION

\subsection{Definitions}

Before the Open Function can be discussed, the terms and concepts which are basic to the EDMF must be given precise definitions. The definitions used in this thesis are consistent with those in [7]. However, they will be found to be less formal and more descriptive.

\subsubsection{Attribute-Value Pair}

The most basic concept which must be defined is that of the attribute-value pair. Let there be two sets, $A$ and V. The elements of A are those terms which are considered as "attributes", and the element of $\mathrm{V}$ are those terms which are considered as "values". Let a third set $D$ be the subset of the Cartesian product $A \times \cdot V$, whose elcments are the ordered pairs of the elements of $\mathrm{A}$ and $\mathrm{V}$. A single element of $D$ is called an attribute-value pair, and intuitively it constitutes the basic element of information. Some examples of attributes, values, and attribute-value pairs are shown in Example 1.

\subsubsection{Record}

A record $R$ is a set of attribute-value pairs which collectively convey some meaningful information. Often these attribute-value pairs are referred to as the fields of the record. An example of $R$, a subset of the set of all attribute-value pairs, is shown in Example 2. The attribute-value pairs in this record convey to the reader information about a book on the subject of public education. 
1a: A set of attributes

$A=\{$ author, year, topic, abstract, text $\}$

Ib: A set of values

$V=\{$ Lieberman, 1960, public education, [the complete abstract of a book], [the complete text of a book]\}

1c: $A$ set $D$ of ordered pairs which are attribute-value pairs

A $\times V \rightarrow D=\{$ (author, Lieberman), (year, 1960), (topic, public education), (abstract, [the complete abstract of a paper]), (text, [the complete text of a paper])\}

Example 1: Examples of attribute, values and attribute-value pairs

$R=\{$ (author, Myron Lieberman),

(title, The Future of Public Education),

(topic, public education),

(publisher, University of Chicago Press),

(year, 1960),

(abstract, [the complete abstract of the book]),

(text, [the complete text of the paper])\}

Example 2: Record of a book on the subject

of public education 


\subsubsection{Keywords}

A record can be characterized by any combination of the attributevalue pairs which are in the record. Due to pragmatic considerations, it would be desirable to have those attribute-value pairs which are short and can be simply expressed, characterize the record. These short attribute-value pairs are called keyworäs, and will henceforth be denoted symbolically by $K_{i}, i=1,2, \ldots n$. Thus we can refer to a record $R$ by referring only to the keywords in $R$. The record in Example 2 can be characterized by the set of keywords shown in Example 3 . In general, the set of keywords of a record $R$ is called an index of the record $R$ and it is usually a proper subset of $R$.

$$
\text { The index of } \mathrm{R}=\left\{\begin{aligned}
& \text { (author, Myron Lieberman), } \\
& \text { (title, The Future of Public Education), } \\
& \text { (topic, public education), } \\
& \text { (publisher, University of Chicago Press), } \\
& \text { (year, 1960) }\}
\end{aligned}\right.
$$

Example 3: The keywords characterizing the record in Example 2

At this point we would like to introduce a notational change for the attribute-value pair. Hereafter an attribute-value pair will be written in the following manner:

$$
\text { Attribute }=\text { Value }
$$


This is the actual notation used in the EDDF for specifying attribute-value pairs.

\subsubsection{Keyword Lists}

Each record is also tharacterized by another parameter which is not part of the actuai information conteined in the record. This unique number is the address of a record, which indicates the whereabouts of the record in the computer storage.

Each keyword $\mathrm{K}_{i}$ in $\mathrm{R}$ may have associated with it the address of another record $R^{\prime}$ which also contains the keyword $K_{i}$. Effectively this address in $R$ "points" to $R^{\prime}$ and for this reason it is called the pointer of $R$ with respect to $K_{i}$ or the $K_{i}$-pointer of $R$. If the record $R^{\prime}$ is non-existent then the $K_{1}$ pointer of $R$ is known as the null pointer. It will be assumed hereafter that every keyword has a pointer associated with it. Thus we see that records containing a common keyword $\mathrm{K}_{1}$ can be linked by these pointers into a chain which is called a $\mathrm{K}_{1}$-list. Putting it more precisely, a $\mathrm{K}_{i}$-list is a chain of records, each record containing the keyword $\mathrm{K}_{i}$, satisfying the following five conditions:

1) Each of the pointers in the $K_{i}$-list are distinct.

2) Each non-null pointer is the address of a record in the $\mathrm{K}_{\mathrm{i}}$-list only.

3) There is one record not pointed to by any other record in the $\mathrm{K}_{i}$-list. This is the beginning of the $\mathrm{K}_{i}$-list.

4) There is one record which has the null pointer; this is the end of the $K_{i}-$ list.

5) For every record in the $K_{i}$-list at the address $a_{n}(n>1)$, there is a sequence of $\mathrm{K}_{i}$-pointers

$$
\left(a_{1}, a_{2}, \ldots, a_{n}\right)
$$




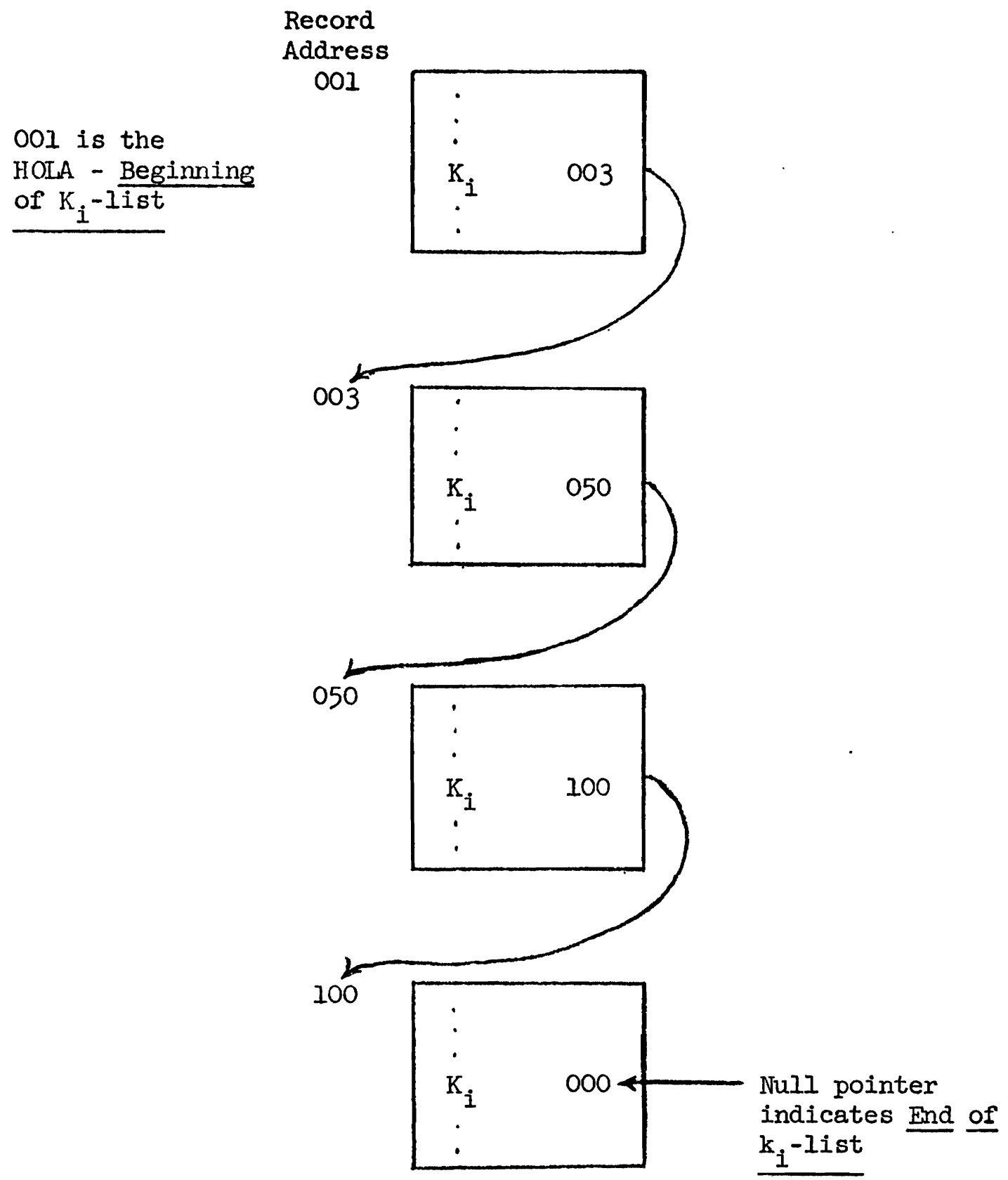

Figure 1: An illustration of a $\mathrm{K}_{i}$-list 
such that:

i) $a_{1}$ is the address of the beginning of the $\mathrm{K}_{1}$-list.

ii) the record at the address $a_{j}$ contains a $K_{i}$-pointer $a_{j+1}$ for $j=1,2, \ldots, n-1$.

This means that for a given $K_{i}$, a record cannot be in more than one $\mathrm{K}_{1}$-list. The address of the first record in a $\mathrm{K}_{i}$-list is known as a Head-of-List Address or HOIA for short, and this term will be used hereafter when referring to the beginning address of any $\mathrm{K}_{1}$-list. In Figure 1, a typical $K_{i}$-list is illustrated, showing the beginning and the end of the list and the pointers which chain the records together. 2.1.5 File and Directory

A file is a set of records which completely contains all the $K_{1}$-lists made up of those records. In other words, a file is a set, whose elements are records, which is the union of all the $K_{i}$-lists which contain the records. The HOLA's of all the $K_{1}$-lists in a given file must be carefully noted and kept separate from the HOIA's of the $\mathrm{K}_{\mathrm{i}}$-lists in another file because the same keyword, but with different meanings, can occur in both these files.

This leads us to the concept of a directory for a file. The directory associated with a file contains the HOLA's of all the $K_{1}-1$ ists in that file. For each keyword $k_{i}$ used in the file, there is one entry in the directory, the form of the entry being shown in Example 4. More precisely, a directory for a file is a sequence of $\mathrm{m}$ such entries where $m$ is the number of different keywords used in the file. 


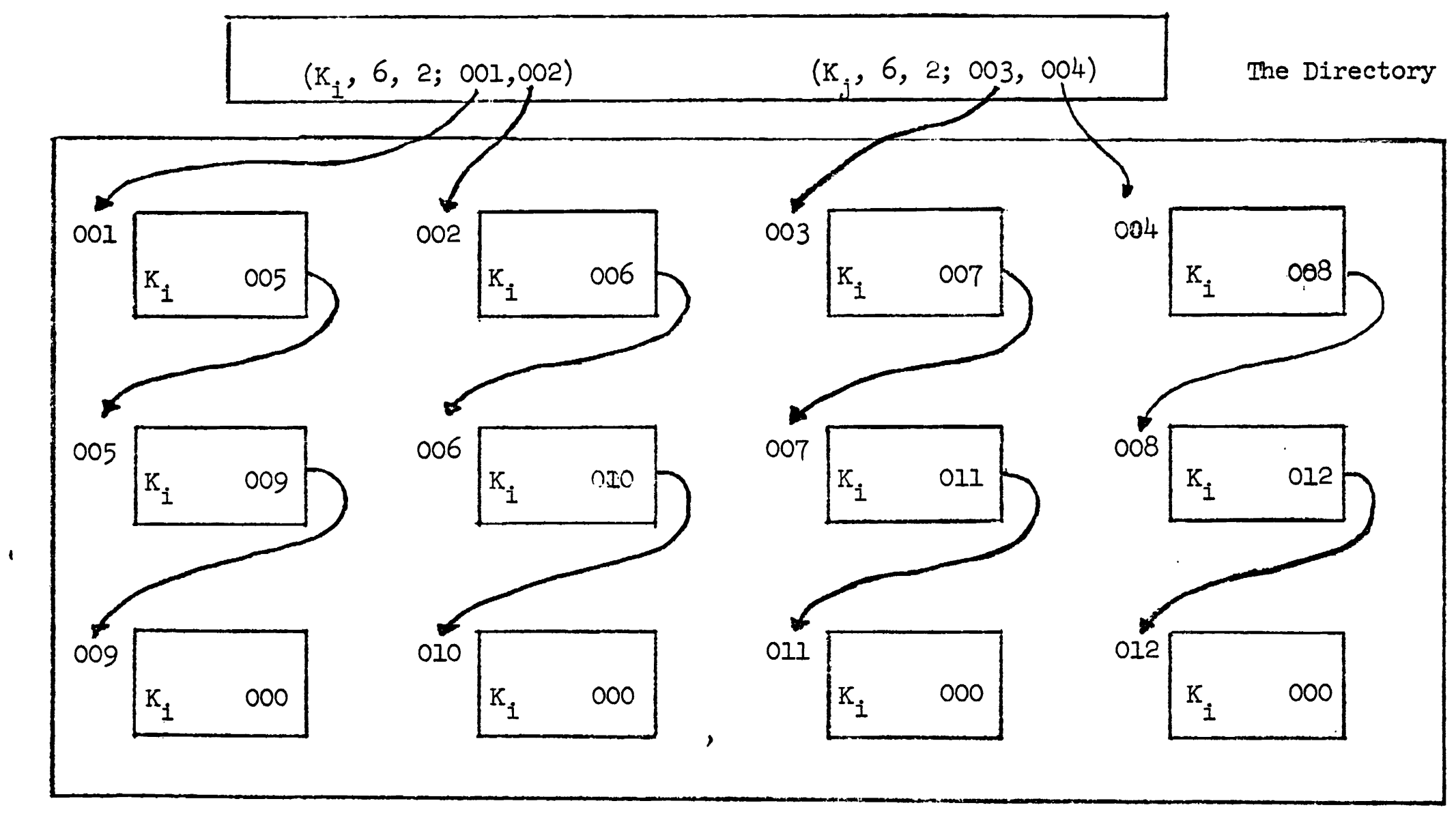

The File

Figure 2: Example of a Generalized File Structure Showing the Logical Relationship Between the Directory Entries and the Keyword Lists 


$$
\begin{aligned}
& \left(K_{i}, n_{i}, h_{i} ; a_{i l}, a_{i 2}, \ldots, a_{i h_{i}}\right) \\
& K_{i}-\text { the } i^{\text {th }} \text { keyword in the file } F . \\
& n_{i}-\text { the number of records in F containing the } \\
& \text { keyword } K_{i} \\
& h_{i} \text { - the number of } K_{i}-\text { lists in } F . \\
& a_{i j}-\text { the HOIA of the } j^{\text {th }} K_{i} \text {-list in } F .
\end{aligned}
$$

Example 4: Format of a directory entry

\subsubsection{Generalized File Structure}

We can now define a generalized file structure as a file with its directory. This file structure is called generalized because it can be shown that many commonly used file structures such as inverted, indexsequential, and multilist are actually special cases of the generalized file structure [8]. An example of a generalized file stmucture is shown in Figure 2.

As was evident in the directory format, there may. be more than one list corresponding to a particular keyword $\mathrm{K}_{i}$, but these lists are mutually exclusive because of the definition for lists presented previously. In other words, a record containing the keyword $\mathrm{K}_{i}$, cannot be in two different $K_{i}$-lists.

However, since a record may have more than one keyword, it may be in more than one keyword list. A record containing the keywords $K_{1}$ and $K_{j}$ (with $1 \neq j$ ), is a member of one $K_{i}$-list and one $K_{j}$-list simultaneously. For example, if a record contains both the keywords AUTHOR = IIEBERMAN and YEAR $=1960$, then that record would be in both an AUTHOR = IIEBERMAN IIst and in -a YFAR $=1960$ list. This is illustrated in Figure 3, where the 


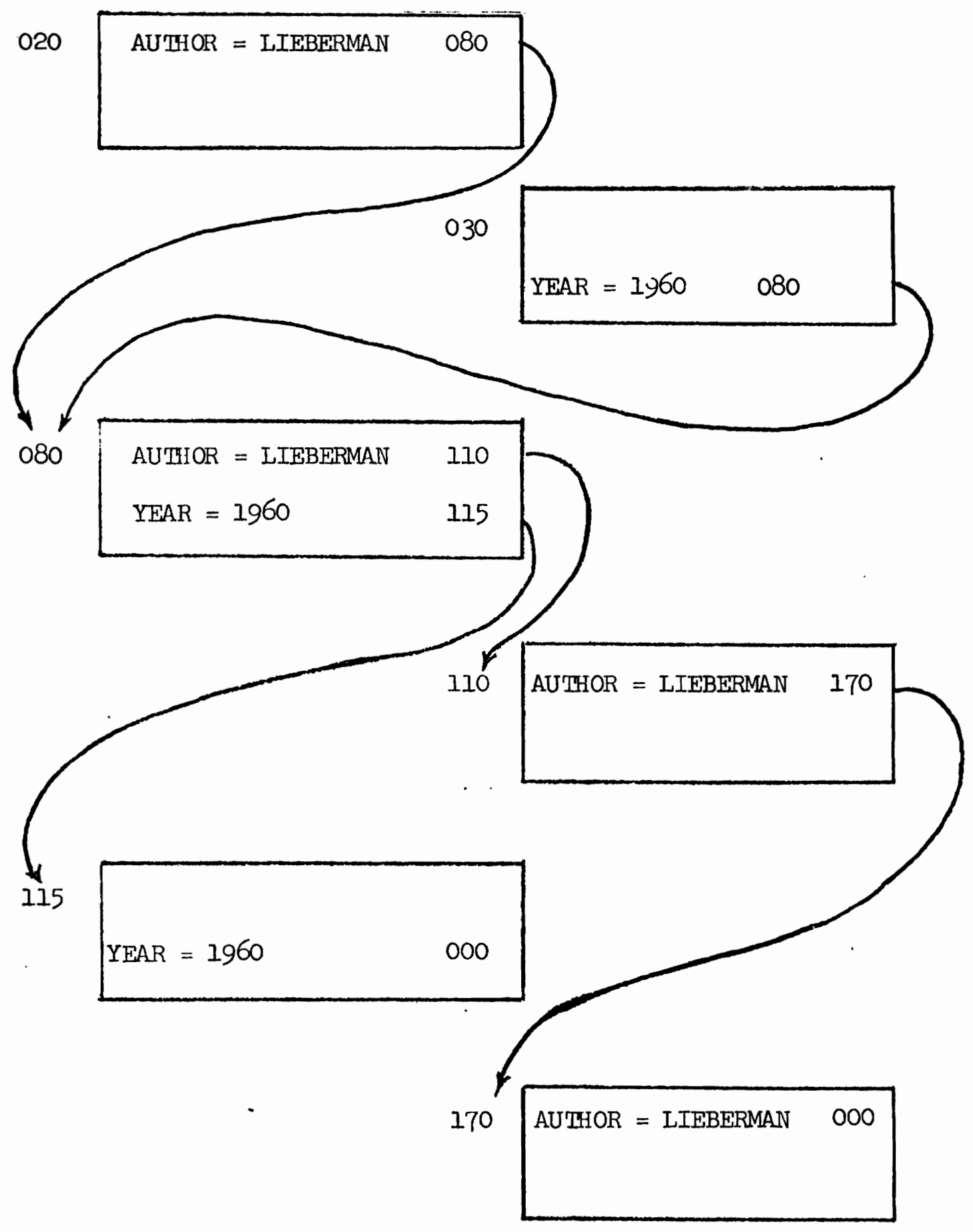

Figure 3: Example of intersecting $K_{i}$-list and $K_{j}$-list $\mathrm{K}_{\mathrm{i}}:$ AUTHOR $=$ LIEBERMAN $K_{j}: \quad Y E A R=1960$ 
AUTHOR = LIEBERMAN list consists of records located at the addresses $020,80,110$, and 170 , and the YEAR $=1960$ list consists of records located at the addresses $030,80,115$.

\subsubsection{Request Description}

When a person accesses a file, rarely does he want to see all of the records in the file. Rather, he usually wants to see only that part of the file which interests him. Such a partition can be accomplished by listing the addresses of the records which he wants. This, however, is cumbersome and requires much research on the user's part to find the addresses of the records in which he is interested. Another way to partition the file would be to describe the records of interest by listing their characterizing keywords in the form of a Boolean expression. This expression is called a user's request description. Using the propositional calculus, any Boolean expression can be uniquely written as a disjunct of conjuncts, known as the Disjunctive Normal Form (DNF). Some typical request descriptions could be

$$
\begin{aligned}
& \text { 4a: } \text { AUTHOR }=\text { MYRON LIEBERMAN } \\
& \text { 4b: } \quad \text { AUTHOR }=\text { MYRON LIFBERMAN } \Lambda \text { YEAR }=1960 \\
& \text { 4c: (AUTHOR }=\text { MYRON LIEBERMAN } \triangle \text { YEAR }=1960) \quad \mathrm{V}(\text { AUTHOR }=\text { HIRSCH) }
\end{aligned}
$$

Example 4: Typical request descriptions

All the request descriptions used in the EDMF will be in Disjunctive Normal Form. 
A record satisfies a user's request description when all the keywords in at least one of the conjuncts of the request description are in the record. A record containing only the keywords $\mathrm{K}_{7}$ asd $\mathrm{K}_{3}$ satisfies the request description containing only one conjunct $\left(K_{1} \Lambda K_{3}\right)$, but does not satisfy $\left(\mathrm{K}_{1} \wedge \mathrm{K}_{2} \wedge \mathrm{K}_{3}\right)$. The problem of finding in a file, the addresses of records which satisfy a user's request description now lies with the EDMF and not the user.

\subsubsection{Entering the EDMF}

There are two ways to enter the EDMF - either through a terminal command or through a system macro. This thesis will discuss only the aspect of the system macro. A discussion of the command entrance can be found in [10].

It was decided that the best way for a non-conversational user to enter the EDMF would be through the use of system macros. Each macro instruction generates a group of assembly language statements. One of the statements generated is a supervisor call. The supervisor call instruction (SVC) enables the program to switch from any state to the Interrupt Control State $\left(\mathrm{P}_{3}\right)$, i.e., the SVC causes an interrupt. It is in the state $\mathrm{P}_{3}$, through the use of the interrupt analyzer, that the supervisor decodes the SVC number and determines which routine should handle the intermupt. Statements that accompany the SVC in the macro expansion supply the necessary parameters for the processing of the user's request. Once the system knows how to respond to the intermpt, it switches to state $\mathrm{P}_{2}$ where interrupt responses are handled. For a diagramatic flow of the above process, see Figure 4. 


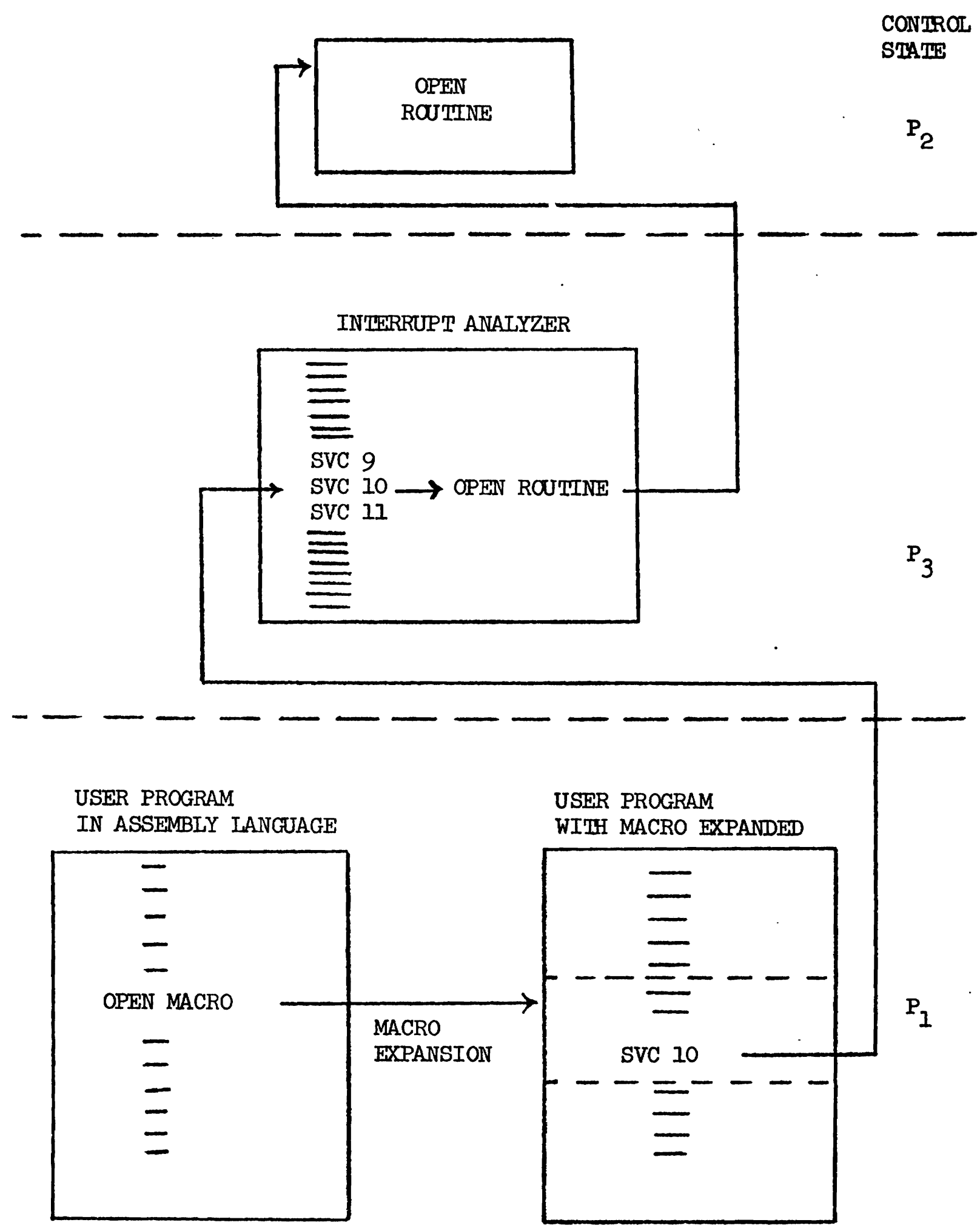

Figure 4: Diagram of Intermupt Handling Process 
Macro instructions are extremely useful since they are located in a macro library accessible to all users. Each time a user writes a macro instmuction, the associated statements and the SVC are generated and incorporated into his program. The only information the user needs to know in using a macro is the proper way of calling it; all the other steps, the generation of instructions and the SVC, are done by the assembler.

Necessary background material has now been discussed and the remaining part of the chapter will devote itself to the open function. 2.2 Purpose of the Open Function

The purpose of the open function is to check the user's access rights to a specified partition of a file, to set up the necessary control blocks for processing the various service requests, and then to return control to the user. Since the open function assembles the necessary system control blocks for all the available service requests, it must be the first function called upon by the user. There are two routines that implement the open function. They are called OPNPROC and FIFDIRSI. (Appendix B.I and B.2)

\subsection{Access Control}

\subsubsection{Introduction}

In any data management facility, the security and integrity of the records are as important as the ease with which processing occurs. A good system is one in which the security precautions are reliable enough to insure file protection while simultaneously not encumbering any of the processing mechanisms. Insuring the integrity of the files encourages users to store their files in the data management facility, and to enlarge the data base. Ease of using the system will encourage frequent use of this data base, leading to an orderly and efficient 
utilization of information storage and dissemination.

\subsubsection{File Level Check}

In the Extended Data Management Facility (EDMF), the protection mechanism operates at three levels corresponding to the logical levels in any file structure. These are the file level, the record level, and the field level. This thesis will discuss only the file level check; a discussion of the other two levels of protection can be found in [4].

In general, and as it presently exists under the ISOS Data Management System (DMS), a file level check is concerned with the security of the file as a whole, and controls any access whatsoever to the file. There are two possible types of file access - either the write, or the read option. If a file has the write option, then a user can update any or all of the existing file records, create new records, and read from the entire file. If, however, the read option is in effect, changes may not be made in the existing file, 1.e., the user may only see the records. The present TSOS DMS protection scheme is an "all or none" type of response; that is, either the entire file is accessible to the user, or access is completely denied and the user's request is terminated. The important point here is that access is dependent on the accessibility of the entire file.

But there certainly are cases when a user should have access to certain portions of a file and not be entirely blocked out. For example, let us suppose that we are dealing with a company's file, named PRODUCTS IN PIANNING (PIP), which is a file of records consisting of information on products currently in the planning stages. Possible products could be televisions, radios, computers, etc. Let us also suppose that a user (call him USER A) has the authority to read all 
the records in this file except those pertaining to computers. Under the current system, access to the file would be denied due to the "all or none" phenomenon. Since USER a is not authorized to reference any of the records pertaining to computers, he is denied access to the entire file.

There are two possible ways to circumvent this problem. One would be to set up a second file which would consist of a subset of the records in the P.TP FIIE and would contain all the PIP records except those pertaining to computers. Now, USER A would have a file that he could access. But, what if there exists a USER B who is allowed to work with all the records in the PIP file except those pertaining to televisions. Do you set up another file for him? This certainly would amount to a duplication of information and a large waste of storage space.

The other and more efficient way of avoiding the "all or none" restriction is by devisıng a method which would allow access only to those partitions of a file that a user is authorized to handle, and block him out of those that are restricted to him. It is in this way that the Extended Data Management Facility handles the problem of file protection. In order to put this method into effect, there must be a way of validating a user's authorızation and secondly, a way of partitioning a file. First, we will discuss the method used to partition a file.

\subsubsection{Partitioning the File}

The expression used to partition a file for the open function is the same type of expression that will be used in requesting the retrieval uf records. It is a logical expression in Disjunctive Normal Form (DNF) where each element of a conjunct is a keyword of the file. This partitioning method is very flexible since it can be used for any file in 
RECORD I

AUTHOR = BROWN, CHRISTY
TITLE = DOIN ALI THE DAYS
PUBLISHER = STEIN AND DAY
YEAR PUBLISHED = 1970

\section{RECORD 3}

AUTHOR = TRAVERS, MIITON TITIE = EACH OTHER'S VICTIMS

PUBLISHER $=$ SCRIBNER

YEAR PUBLISHED $=1970$
RECORD 2

AUTHOR = WEITZ, $\mathrm{J}$
TTTEE = THE VAIUE OF NOTHING
PUBLISHER = STEIN AND DAY
YEAR PUBLISHED = 1970

RECORD 4

$A U T H O R=R A N D, A Y N$

TITHE = WE THE LIVING

PUBLISHER = RANDOM HOUSE, INC.

YEAR PUBLISHED $=1936$

RECORD 5

AUTHOR = RAND, AYN
TITIE = ATIAS SHRUGGED
PUBLISHER = RANDOM HOUSE, INC.
YEAR PUBLISHED = 1957

Figure 5: Library Catalogue File 
the system. In addition, it does not require that the user know the actual addresses of those records that he is interested in.

For purposes of illustration, let us say we had a library catalogue file with only the five records that appear in Figure 5. One partition of this file would be those records which refer to books that were publisıed by Random House, Inc. in 1936. A DNF description would appear as:

(PUBLISHER = RANDOM HOUSE, INC. $\Lambda$ YEAR PUBLISHED $=1936)$ Only record 4 satisfies this description.

A second partition would be those books published by Stein and Day and those published by Random House, Inc.

(PUBLISHER = STEIN AND DAY $\Lambda$ PUBLISHER = RANDOM HOUSE, INC.) Records 1, 2, 4, and 5 satisfy this description.

A third partition might be those books published by stein and Day in 1970 and books that were published in 1957

$$
(\text { PUBLISHER }=\text { STEIN AND DAY } \Lambda \text { YEAR PUBLISHED }=1970) \quad \mathrm{V}
$$

$$
\text { (YEAR PUBLISHED }=1957 \text { ) }
$$

The satisfactory records here are 1,2 , and 5.

2.3.4 User's Authority Item

In order to validate a user's authorization to access a file, the system must obtain information concerning the user's access rights to that particular file. This information could be stored in a record at the head of each file. This type of security system would be fileoriented.

The EDMF does not take this approach but rather a user-oriented one. The EDMF ureates a system file which is known as the Authority Item file. This file consists of a set of records with one record for 
each user. Each record is an individual user's authority 1tem (UAI). The UAI's contain information pertaining to the user's access rights to the files maintained by the system. Therefore, by examining a specific user's authority item the system can determine to what degree the user is allowed to utilize the existing files.

There are two advantages to this user-oriented type of protection. First of all, since all authorizing information is stored in a system file, it is better protected than if it were stored at the head of a user file. In this case, only the system is allowed to handle the Information, thereby making the chance of user intervention very slight. The second advantage is that updating authority information is quite routine. The user's authority information is all stored in one place the User's Authority Item. Since the system file's internal format is consistent with the internal format of the user files, the same retrieval and updating routines may be used. Additional processing routines for whe authority items are unnecessary, consequently making the most efficient use of the EDMF retrieval and updating routines.

Upon the issuance of a call to the open function, the user's authority item is referenced. If access to the requested partition of a file is granted, processing continues with the necessary system control blocks being established; if access is denied, the system returns control to the user with an explanatory message.

\subsection{Control Blocks}

When access to a file is granted, the open function makes entries into two important system control blocks. One is the service status Block and the other is the File status Block. 


\subsubsection{Service Status Block}

The Service Status Block (SSB) contains status information about every file processed by a user during a TSOS session [9]. It is useroriented, which means that each user of the system has his own SSB, containing information relevant to only those files which he is using. The SSB is created when a user logs on to ISOS, remains with the user's task throughout its existence in TSOS, and is destroyed when the user logs off.

The purpose of the SSB is to eliminate duplicate retrievals of control information about the user files. It is certainly more worthwhile to use a small amount of storage space to hold the control information, than to spend processing time to re-retrieve it. The problem can best be illustrated as follows. Suppose a user opens a file under the system and then tries to retrieve some information. Due to the structure of the ISOS system, the retrieve request, as far as the system is concerned, is a separate entity from the previous open. This means that the processing routine for the retrieval must be able to check that the requested file has been previously opened. For security reasons, this information is kept in the SSB in privileged system memory. The first file to be opened by a user results in information being stored in the SSB section created during logon. All subsequent file openings cause additional SSB sections (one per each file partition) to be chained to the initial section in a linked list. Thus, each user's SSB can grow as the number of files or their partitions referenced during a session grows. Consequently, there is one SSB section for each file partition that is requested. 
One important point to note is that a file need not be opened to have an entry in the user's SSB. (See Chapter 4) What is relevant is whether or not the file's control information is already in storage. This could be the case if the file had been previously opened and then closed. If the file's control information is in storage, then addresses to this information can be found in the SSB. This procedure saves unnecessary retrievals and the waste of duplicate processing time.

\subsubsection{File Status Block}

The File Status Block (FSB) contains status information about every file that is currently being processed by any user during a ISOS session. It is file-oriented which means that an entry is made in the FSB each time a user opens some partition of a file. This FSB entry is established immediately after the SSB block is created. Each file referenced during a TSOS session has its own linked list whose entries include the following information: the user's Id, the type of open requested, and the partition of the file that has been opened.

The purpose of the FSB is to establish priorities relative to the use of the file. The problem can be illustrated as follows. Let us suppose that two users, USER A and USER B, want to work with FILE 1 . USER A wants to read from the file while USER B wants to update it. Let us also assume that USER A issued his open request first. Then the system, by referencing the FSB, could establish that USER A has the priority and permit him to read from the file, while blocking USER B from updating it. Otherwise, USER A could possibly receive erroneous information. 
Now let us luok at an example where partitioning plays a part. Going back to our library catalogue example (Figure 5), suppose that USER A wants to update that partition of the file which satiofies the DNF description

$$
\text { PUBLISHER = RANDOM HOUSE, INC. } \Lambda \text { YEAR PUBLISHED }=1936
$$

Recall that record 4 is the only member of this partition. Let us also suppose that USER B wants to read from the partition satisfying

(PUBLISHER = STEIN AND DAY) $V$ (PUBLISHER = RANDOM HOUSE, INC. $)$ The satisfactory records are $1,2,4$, and 5. Again, USER A issued his open request first and therefore had priority. But, the only requested record that USER A and USER B have in common is record 4. The system references the FSB chain for the library catalogue file to determine the position of USER A's entry. USER A's entry precedes USER B's in the chain and therefore, $A$ has priority. USER A is allowed to update record 4 while USER $B$ is blocked out. But, USER B is allowed to read records 1,2 , and 5 .

The individual FSB entries remain in the file list until the user closes the file. It is at this time that the user no longer holds any position in the priority list and therefore his FSB entry is removed.

\subsection{Return to User}

After both the SSB and FSB have been constructed, the system returns control to the user. If the user entered the system via an SVC call issued from a program, then control is returned to the instruction following the SVC call. If, however, entry was from a command, then control is returned to the Terminal Command Processor which returns control to the user at the terminal. The user, now in control, is free to continue the execution of his program or call upon any other functions of the EDMF. 


\subsection{The EUMF's OPN Macro}

One way of initiating the open function (see Sect. 2.1.8) is through the use of the EDMF macro named OPN. The OPN macro has three required parameters. One is the requested file name. A second is the type of open requested, i.e., either update or read. The third one can be either the actual partitioning description or the address of where this description can be found. For a more detailed discussion of this macro, please see Appendix A.I. 
CHAPTTER 3

THE RETRIEVAL INITIALIZATION FUNCTION

\subsection{Purpose}

The main purpose of the Retrieval Initialization (RI) function is to optimize the retrieval processing and to obtain necessary information for the actual record retrieval. This information includes prime keywords, ISAM keys and Record Format numbers. But, before this information is obtained, the control blocks that were established by the open function must be checked.

\subsection{Control Blocks}

In order for the processing of the actual retrieval mechanism to start, the user must have previously issued a satisfactory open request. If this was the case, then there is an SSB entry for that partition of the file that he wishes to reference. As the first step in the processing of the RI function, it checks the SSB entries. If the required entry is found, then a ISOS DMS open macro is issued. If the SSB entry does not exist, the processing of the retrieval initialization function is terminated and an explanatory error message is returned to the user.

\subsection{DMS Open}

The TSOS DMS open must precede any call for the primitive storage and retrieval routines. Without the DMS open, the primitive routines cannot access the file. The primitive routines actually perform, through the data management facilities provided by the operating system, the input and output of records for other system components. These routines handle the actual reading and writing of the data records, the manipulation of the files' directories, and the generation and updating of the records and directories of the files. 
In processing the retrieval optimization algorithm, the $\mathrm{RI}$ function needs to reference the file's directory. In order to use the directory routines, a DMS open must be issued. This brings us to an important point relative to the issuance of the DMS open. There are two possible times that the DMS oper. macro could be 1ssued: either during the processing of the EDMF's open function or during the RI function. It was decided that the best time would be during the processing of the RI function. This decision was made for the following reason. Once a DMS open is issued, entry into the opened file is blocked to other users until a DMS close is issued. The routines that actually require a DMS open, that is, the primitive routines that handle the requested file's directories and/or records, are not needed until the RI phase of the EDMF. Therefore, the issuance of a DMS open during the EDMF's open function would block the requested file from other users for a longer period of time than necessary.

\subsection{Retrieval Optimization}

In an attempt to make the retrieval system as efficient as possible, an optimizing retrieval method was needed to minimize the time required to process a retrieval request. The algorithm chosen for the optimization phase was part of the General Retrieval Algorithm as suggested by D. Hsiao and F. Harary in their paper titled "A Formal system for Information Retrieval From Files" [8]. The first step of the algorithm involves the selection of prime keywords from the user's DNF description of requested records. 


\subsection{Prime Keywords}

As you recall, each user's DNF request description consists of one or more conjuncts whose elements are keywords of the fije. For example, a possible DNF description could be

$$
\left(\mathrm{K}_{1} \wedge \mathrm{K}_{2} \wedge \mathrm{K}_{3}\right) \vee \mathrm{K}_{4}
$$

where the $K_{i}$ are keywords of the file. For the purposes of this example let us say that

$$
\begin{array}{cl}
\mathrm{K}_{1}: & \text { AUTHOR = SMITH } \\
\mathrm{K}_{2}: & \text { YEAR }=1964 \\
\mathrm{~K}_{3}: & \text { TOPIC = MATH } \\
\mathrm{K}_{4}: & \text { AUTHOR }=\text { COHEN }
\end{array}
$$

our description would then appear as follows:

$($ AUTHOR $=$ SMITH $\Lambda$ YEAR $=1964 \Lambda$ TOPIC $=$ MATH $) \quad V \quad(A U T H O R=$ COHEN $)$

Associated with each of the keywords. In the file's directory is the number of records in the file in which the keyword appears. The prime keyword is defined as that keyword of the conjunct which appears in the least number of records in the file. Going back to our example: let $\mathrm{N}$ be the number of records in which a keyword appears, and let the following correspondence be established:

$\begin{array}{clr}\text { Keyword } & \underline{\mathbf{N}} \\ \mathrm{K}_{1} & \text { AUTHOR }=\text { SMITH } & 10 \\ \mathrm{~K}_{2} & \text { YEAR }=1964 & 15 \\ \mathrm{~K}_{3} & \text { TOPIC }=\text { MATH } & 2 \\ \mathrm{~K}_{4} & \text { AUTHOR }=\text { COHEN } & 15\end{array}$

For the first conjunct $\left(K_{1} \wedge K_{2} \wedge K_{3}\right), K_{3}$ would be the prime keyword since only 2 records exist in the file that contain TOPIC $=$ MATH. The prime keyword for the second conjunct must be $K_{4}$ since it is the sole 
member of the conjunct.

Now, how does the designation of prime keywords relate to optimizing the retrieval? First of all, we only want to retrieve those records that satisfy each conjunct. Since a record can only satisfy a conjunct by containing every keyword in the conjunct, all satisfactory records must contain the prime keyword. Thus searching the file using the prime keyword, i.è., actually retrieving the least number of records that could possibly satisfy the expression, minimizes the costly time of actual retrieval and thereby results in an optimum retrieval scheme.

The selection of the prime keywords is accomplished in a routine called REIRIEVE. The RETRIEVE routine also picks up the ISAM keys.

\subsection{ISAM Keys}

In order for the primitive routines to actually retrieve records, they must know the locations of the requested records. The address of the record location depends on the type of access method used to store the records. The EDMF utilizes RCA's TSOS Data Management System Indexed Sequential Access Method (ISAM) for device level input/output. In this access method, each record of a file is assigned a key, a number from 0 to $99,999,999$. This number allows one to refer to a record by a logical address (its ISAM key) instead of a physical disk address [6]. Once the prime keyword for a conjunct is established, the REIRIEVE routine must pick up the corresponding ISAM keys for the actual record retrieval. Again, the RETRIEVE routine returns to the directory. Associated with each keyword in the directory are the head of list addresses (HOLA). These head of list addresses are ISAM keys whose records contain that keyword [3]. The RETRIEVE routine then makes a list of all HOLA's that correspond to the prime keywords of the description. Once 
this is finished, corresponding record format numbers must be established.

\subsection{Record Format Numbers}

One of the major design criteria used in determining the form of the EDMF records and their control information is as follows. As much information as possible should be removel from the record and stored as file control information. This prevents duplication of information appearing in many records, thus making files smaller. In other words, general structural information is centralized into one file control block rather than decentralized in the individual records.

When records are collected into a file, the usual case is that all records have similar attributes, because they contain the same type of information. For example, all records in a file of library books are likely to contain the attribute "Author". Thus it is reasonable to expect that there are only a limited number of different attributes in a file. In order to save space in the file, the attributes are removed from the records and placed in a file control block called the Record Format Block (RFB). Associated with each attribute in the RFB is a format number. It is this format number and not the entire attribute that is stored in the record [9]. A detailed specification of the RFB can be found in Appendix C.3 •

After a record has been retrieved from disk, it is necessary for the record validating function [4] to determine if it satisfies the user's description. In order to do this, it must check to see if all the keywords of a conjunct can be found in the record. Since only the format numbers and not the actual attributes are stored in the record, it is necessary to determine the corresponding format numbers before the record validating function can operate. The program that performs this service 
for the RI function is called FORPROG. It checks the attributes in the user's request description against those in the RFB and then makes a list of corresponding format numbers.

\subsection{Control Passed to the File Searching Function}

Once the lists of prime keywords, ISAM keys, and Record Format numbers are established, the work of the Retrieval Initialization function is finished. The lists and supervisor control is then passed to the File Searching Function [4]. After the File Searching, Record Validating and Record Formatting functions [4] have completely processed the request, the system initiates a DMS close macro. The file can now be actively accessed by other users subject to the priorities established in the File Status Blocks.

\subsection{The EDMF's RETR Macro}

One entrance to the Retrieval Initialization function is through the use of the EDMF's RETR macro. This macro has six possible parameters. Of these six parameters at least three and not more than five may appear in one macro call. Two of the required parameters are the file name and the output specification. The third required parameter can be either the user's retrieval request description or the address where this description can be found. The fourth parameter, which is optional, is the maximum number of satisfactory records that the user wants retrieved. If this parameter is omitted, all the records satisfying the request description will be outputted to the user. The fifth parameter would be a label. For a more detailed discussion of the RETR macro, see Appendix A.2. 
CHAPTER 4

THE CLOSE FUNCTION

\subsection{Purpose}

The purpose of the close function is to remove a user's priority hold over a specified partition of a file. A user initiates the EDMF's close function when he no longer desires to work with the partition of a file that he had previously opened. The close function makes necessary changes in the control blocks, the SSB and FSB, to indicate that the user has finished all processing of the specified partition of the file. Once this has been done, the user no longer has access to the partition. If he wishes to work with it again, he must re-initiate the EDMF open function. The close function is therefore the last FDMF function that a user would call upon. The routine that implements the close function is called CLSEPROC. (Appendix B.6)

4.2 Control Blocks

During the processing of the open function, a Service Status Block and a File Status Block were created (see Section 2.4). The FSB entry established for the user a position in a priority list relative to the use of the specified file partition. Now that the user has finished working with that partition, he should not maintain his position in the priority list. He no longer has the right to block out other users from accessing the records of the partition. Therefore, the system removes his FSB entry from the priority list and also indicates in the corresponding SSB entry that the EDMF close function has been referenced and that the partition is not open for his use. 


\subsection{Return to User}

After both the FSB and SSB have been updated, the system returns control to the user. The user is now free to continue processing any other files that he had opened, initiate the EDMF open function for another file partition or terminate his session.

4.4 The EDMF'S CLSE Macro

One entrance to the close function is through the use of the EDMF's CLSE macro. This macro has three possible parameters. Of these three parameters at least one, and not more than two, may appear in one macro call. The required parameter is the file name. The optional one can be either the actual partitioning description or the address of where this description can be found. If the optional parameter is omitted, the system assumes that the user wants to close out all the partitions of the specified file that he had opened. Otherwise, only the specified partition is closed. For a more detailed discussion of the CLSE macro, see Appendix A.3 • 


\section{CHAPTER 5}

\section{SUMMARY}

The Extended Data Management Facility (EDMF) was implemented to provide a general purpose data management system for the orderly accumulation and dissemination of information. The EDMF utilizes a generalized file stmacture and an efficient retrieval algorithm for efficient data management.

It was the purpose of this thesis to discuss a portion of the Supervisor's task in the EDMF. The task is to direct the Facility's handling of a user's request and by so doing, the Supervisor assumes the oles of "doorman", "foreman", "administrator", and "dispatcher". In order for the Supervisor to fulfill its task and satisfy its roles, it performs five main functions: Access Control, Retrieval Initialization, File Searching, Record Validating, and Record Formatting. The last three functions, File Searching, Record Validating and Record Formatting, are the functions which partially fulfill the roles of "foreman", "administrator" and "dispatcher". They are discussed in detail in [4]. This thesis has discussed the Access Control and Retrieval Initialization Functions with special emphasis on the Retrieval Optimization subfunction.

These functions fulfill the role of "doorman" and partially those of "foreman" and "administrator". As you remember, macro instructions are used as the "doorman's" entrance into the request handling routines. The Prime Keyword search (Retrieval Optimization subfunction) of the user's DNF Boolean request expression is the "foreman's" method of optimizing the retrieval strategy. The "administrator's" role is fulfilled by the Access Control function. It maintains the security control over file access by checking the user's authority item before processing his request. 


\section{BIBLIOGRAPHY}

1. Chen, T., et al., "An Interim Report on the Implementation of the Integrated Facility," Project Report, The Moore School of Electrical Engineering, University of Pennsylvania, April, 1970.

2. Corwin, B., et al., "An Integrated Information Storage, Retrieval and Dissemination Facility," Project Report, The Moore School of Electrical Engineering, University of Pennsylvania, June, 1969.

3. Desiato, B., "Directory Constructing and Decoding in a Generalized File Structure," M.Sc. Thesis, The Moore School of Electrical Engineering, University of Pennsylvania, work in progress.

4. Ets, A. R., "The File Searching, Record Validating and Record Formatting Functions of the Supervisor for an Extended Data Management Facility," M.Sc. Thesis, The Moore School of Electrical Engineering, University of Pennsylvania, August, 1970.

5. Gana, J., "A Command and Query Language Assembler for an Extended Data Management System," M.Sc. Thesis, The Moore School of Electrical Engineering, University of Pennsylvania, work in progress.

6. Horton, M., "Reading, Writing, Creating and Updating Records and Files in a Generalized File Structure," M.Sc. Thesis, The Moore School of Electrical Engineering, University of Pennsylvania, work in progress.

7. Hsiao, D. K., "A File System for a Problem Solving Facility," Ph.D. Dissertation, The Moore School of Electrical Engineering, University of Pennsylvania, May 1968.

8. Hsiao, D. K. and Harary, F., "A Formal System for Information Retrieval From Files," Communications of the ACM, Vol. 13, No. 2, February, 1970. 
9. Manola, F., "An Extended Data Management Facility for a General Purpose Time Sharing System," M.Sc. Thesis, The Moore School of Electrical Engineering, University of Pennsylvania, work in progress.

10. McDonald, J., "A Command and Query Language Interpreter for an Extended Data Management System," M.Sc. Thesis, The Moore School of Electrical Engineering, University of Pennsylvania, August, 1970.

11. Wexelblat, R., "The Development and Mechanization of a Problem Solving Facility," Ph.D. Dissertation, The Moore School of Electrical Engineering, University of Pennsylvania, December, 1965. 


\section{APPEIVIX A}

MACROS

\section{A.1 Open Macro}

Name: $\quad \mathrm{OPN}$

Type: Keyword

Four possible keywords - miximum of three permissable

at one time - minimum of two required.

\section{Required}

1) FIIENAM - name of the file (up to 54 characters)

2)*(a) DESCRIP - the actual partitioning logical

expression in DNF form (up to 127

characters, due to the system's

restriction on the length of para-

meters). Single quotes must enclose

the expression and any internal quotes

or ampersands must be doubled. See

the examples.

*(b) DESADDR - this parameter is mnemonic for description address and it must be used when the desired DNF partitioning expression is longer than 127 . characters. This necessitates the placement of the logical expression in an area external to the macro and it is referenced by a symbolic address. 
Optional

1) TYPE - the type of open requested

(a) READ - can only read from the file. Default case.

(b) UPDATE - can read and write to the file.

Examples of Macro Calls

1) OPN FILENAM=\$HORTON MUTTES3, TYPE=READ, DESCRIP=' $A U T H O R=$ BENNET'

2) OPN FIIENAM=MULTTES3, TYPE=UPDA TE, DESADDR=LOGEXP1

LOGEXPI DC C'MONTH=MAY \&\& YEAR=1965' 'OR' ' KEY PHRASES=INFORMATION STORAGE AND RETRIEVAL \&\& PUBLISHER=THE MOORE SCHOOL OF ELECTRICAL ENGINEERING OF THE UNIVERSITY OF PENNSYLVANIA'

Note: * - Only one of these may be used in one macro call.

A.1.1 Generated Parameter List

The OPN macro generates a parameter list whose address is placed in Register $I$ and which is passed on to a handling routine via an SVC call. The generated parameter list has the following format:

$$
\begin{array}{cl}
\text { Bytes } & \multicolumn{1}{c}{\text { Content }} \\
0-1 & \text { Length of file name } \\
2-55 & \text { File name (left justified with spaces) } \\
56 & \begin{array}{c}
\text { Code for type of open } \\
X^{\prime} 42^{\prime}-- \text { Read } \\
X^{\prime} 43^{\prime}-- \text { Update }
\end{array} \\
57-59 & \text { Address of partitioning logical expression }
\end{array}
$$


$60-63$

$64-190$

191
Length of partitioning logical expression

Partitioning logical expression if included in macro

Code for presence of partitioning description

$X^{\prime} 00{ }^{\prime}$-- No description

$X^{\prime} F F^{\prime}$-- Description present 


\section{A.2 Retrieval Macro}

Name: RETR

Type: Keyword

Six possible keywords - maximum of five permissable

at one time - minimum of three required.

$\underline{\text { Required }}$

1) FILENAM - name of the file (up to 54 characters)

2) OUTSPEC - output specification (up to 10 characters)

(a) CORE - output is in special core format [4] in core to be used by program

(b) COUNT - the system returns with the number of satisfactory records and not the actual records

(c) PRINT - output is sent to the printer

(d) TMY - output sent to teletype. Default case. 3) *(a) DESCRIP - the actual partitioning logical expression in DNF form (up to 127 characters, due to the system's restriction on the length of parameters). Single quotes must enclose the expression and any internal quotes or ampersands must be doubled. See the examples.

*(b) DESADDR - this parameter is mnemonic for description address and it must be used when the desired DNF partitioning expression is longer than 127 
characters. This necessitates the placement of the logical expression in an area external to tise macro and it is referenced by a symbolic address.

Optional

1) RECNO - the number of desired records satisfying the description. If this parameter is omitted, all the records satisfying the request will be presented to the user.

2) LABEI - name associated with RETR macro will be used in a CONTINUE [9].

Examples of Macro Calls

1)

RETR FILENAM=MLTTESI, RECNO=10, DESCRIP=' $A U T H O R=S M I T H ~ \& \&$ YEAR=1964 ' 'OR' ' TOPIC=IISP' , OUTSPEC=PRINT

2) RETR FILENAM=MULTTES3, OUTSPEC=CORE, DESADDR=LOGEXP2, IABEL= AGAIN

3) RETR FILENAM=MULTIESI, OUTSPEC=COUNT, DESADDR=LOGEXP2

LOGEXP2 DC C'AUTHOR=MANOLA \&\& YEAR=1970 \&\& TOPIC=INFORMATION STORAGE AND REIRIEVAL \&\& PUBLISHER=THE MOORE SCHOOL OF ELECTRICAL ENGINEERING OF THE UNIVERSITY OF PENNSYLVANIA ' 'OR' ' TOPIC=MA THEMATICS'

Note: *- only one of these may be used in one macro call. 


\section{A-6}

A.2.1 Generated Parameter List

The RETR macro generates a parameter list whose address is placed

in Register $I$ and which is passed on to a handling routine vic an SVC

call. The generated parameter list has the following format:

\begin{tabular}{|c|c|}
\hline Bytes & Content \\
\hline $0-1$ & $\begin{array}{l}\text { Number of requested records to be } \\
\text { retrieved }\end{array}$ \\
\hline & X'0000' -- All records. Default case. \\
\hline $2-6$ & $\begin{array}{l}\text { Output specification. CORE, COUNT, PRINT } \\
\text { or TTY. }\end{array}$ \\
\hline $7-11$ & Label \\
\hline $12-13$ & Length of file name \\
\hline $14-67$ & File name (left justified with spaces) \\
\hline 68 & $\begin{array}{l}\text { Function code } \\
X^{\prime} 22^{\prime} \cdots \text { Retrieval code }\end{array}$ \\
\hline $69-71$ & Address of logical expression \\
\hline $72-75$ & Length of logical expression \\
\hline $76-202$ & Logical expression if included in the macro \\
\hline
\end{tabular}




\section{A.3 Close Macro}

Name: CLSE

Type: Keyword

Three possible keywords - maximum of two permissable at one time - one required.

Required

1) FILENAM - name of the file (up to 54 characters) Optional

1)*(a) DESCRIP - the actual partitioning logical expression in DNF form (up to 127 characters, due to the system's restriction on the length of parameters). Single quotes must enclose the expression and any internal quotes or ampersands must be doubled. See the examples.

*(b) DESADDR - this parameter is mnemonic for description address and it must be used when the desired DNF partitioning expression is longer than 127 characters. This necessitates the placement of the logical expression in an area external to the macro and it is referenced by a symbolic address. 
Examples of Macro Calls

1)

CLSE FIIENAM=\$HORTON $\cdot$ MULTIES3

2)

CLSE FIIENAM=\$HORTON . MLTTES3, DESCRIP = 'AUTHOR=BTNNET'

3)

CLSE FIIEENAM=MUTTES3, DESADDR=LOGEXP3

LOGEXP3 DC

$C^{\prime}$ MONTH=MAY \&\& YEAR=1965 ' $O 5^{\prime}$ ' KEY PHRASES=INFORMATION STORAGE AND RETRIEVAI \&\& PUBLISHER=THE MOORE SCHOOL OF ELECIRICAL ENGINEERING OF THE UNIVERSITY OF PEINNSYLVANIA'

Note: * - Only one of these may be used in one macro call.

\section{A.3.1 Generated Parameter List}

The CLSE macro generates a parameter list whose address is placed in Register $I$ and which is passed on to a handling routine via an SVC call. The generated parameter list has the following format:

$$
\begin{aligned}
& \text { Bytes } \\
& \text { Content } \\
& 0-1 \quad \text { Length of file name } \\
& \text { 2 - } 55 \text { File name (left justified with spaces) } \\
& 56 \quad \text { Code for type of close } \\
& X^{\prime} 48^{\prime} \text {-. Close all partitions of the } \\
& \text { file } \\
& X^{\prime} 49^{\prime}-- \text { Close only the specified } \\
& \text { partition } \\
& 57 \text { - } 59 \text { Address of partitioning logical expression } \\
& 60-63 \text { Length of partitioning logical expression } \\
& 64 \text { - } 109 \text { Partitioning logical expression if } \\
& \text { included in the macro }
\end{aligned}
$$




\section{APPENDIX B}

ROUTINES

\section{B.I Routine OPNPROC}

The OPNPROC routine is the first of two routines that implement the Open Function of the EDMF. This routine checks the user's access rights to the specified partition of a file and sets up the SSB and FSB control blocks.

\section{B.I.I Entry Points}

OPNPROC has three entry points. The entrance via an SVC call is at OPNPROC while the command entrance is at COMDOPN. The FIFBLOCK entrance is used when only the FCB for the File of Files (FIF) is needed.

\section{B.1.2 Exit Points}

There is only one exit point for this routine. It begins at BRETURN where control is returned to the calling program.

\section{B.1.3 External Subroutine Calls}

There are eight external subroutines that may be called upon by OPNPROC. One is AIRETR which retrieves the user's authority item. A second is AUTHCHK which checks the user's access rights to the specifled partition of a file. A third is to the location ESQCAT to obtain the task number. A fourth external subroutine is FIFDIRSI. FIFDIRSI is used to, retrieve the File Information Block (FIB) for the specified file. The following three are entry points in the SSBOPTR routine [9]. SSBACQR is used to obtain the SSB chain for a specified user. SSBLOGON is used to establish the SSB chain if it has not already been done and SSBGTNU is used to obtain a new SSB block to link to the user's SSB chain. 
The eighth external subroutine is FSBOPIR. This subroutine is used to establish the FSB entries. The DSECTS that are associated with the SSBOPTR and FSBOPTR routines are the following:

$\underline{\text { Name }}$

SSB

SSBHDR

SSBUAI

SSBFTF

SSBTXT

SSBB'NAM

SSBCL

SBHIB

SSBFCB

SSBDTBIN

SSBDTAB

SSBCREC

SSBFSB

SSBCTL6

SSBPTR

FSB

I'SBUSRID

PSBCL

FIBDSADR
Bytes

DSECT

$$
0-7
$$$$
0-3
$$$$
4-7
$$$$
8-91
$$$$
8-63
$$

$64-67$

64

65

$66-67$

$68-71$

$72-75$

76

$77-79$

$80-83$

$84-87$

88

$89-91$

DSECT

$$
0-7
$$

$8-11$

$12-15$
Content

SSB Header

Address of User's Authority Item

Address of FCB for File Information File

SSB text

2 bytes - length of file name

54 bytes - file name

Control Information

Type of request

Indicator - EDMF open

Unused

Address of File Information Block (FIB)

Address of File Control Block (FCB)

Open description indicator

Address of user description block

Address of Core Format of the record

Address of F'ile Status Block

Control Information for pointer

Pointer to next SSB block

User Identification

Control Information

Address of user's partitioning description 
Name

FSBLTBLK

FSBCTRL

FSBNTBLK
Bytes

$$
16-19
$$

20

$21-23$

Content

B.1.4 Input Parameter List

The address of the input parameter list (PARAMOP) must be in Register $I$ and Register 13 must contain the address of the calling routine's save area.

Name

Bytes

Content

PARAMOP

DSECT

FLNMLN

$0-1$

Length of file name

FINAME

$2-55$

File name (left justified with spaces)

FUNCODE

56

Code for type of open requested

IOGEXPAD

$57-59$

Address of partitioning logical expression

LNLOGEXP

$60-63$

Length of partitioning logical expression

LOGEXP

$64-190$

DESCODE

191

Partitioning logical expression if included in OPN macro

Code for presence of partitioning description

\section{B.1.5 Register Conventions}

The registers in OPNPROC are assigned in the following manner:

$\underline{\text { Register }}$

0

1

2

3
Utilization

Not used

Address of parameter list given to called subroutine. Miscellaneous use.

Miscellaneous use.

Base for OPNPROC

Miscellaneous use 
$\underline{\text { Register }}$

6
Utilization

Address and base of SSB

Counter for number of charactens in User Id. Miscellaneous use.

Miscellaneous use

Address of current SSB block

Address and base of SSBTEXT

Length of requested file name

Address and base of OPNPROC work area

Address and base of input parameter list (PARAMOP)

Address of OPNPROC save area

Return address in OPNPROC

Subroutine call address. Error codes.

\section{B.1.6 Internal Work Area}

The internal work area (OPENRQ) used by OPNPROC also contains the parameter lists for some of the routines called by OPNPROC. The DPLISTA list is passed to AUTHCHK while PAROPEN is passed to FIFDIRSI. The work area has the following format:

$\begin{array}{lll}\begin{array}{l}\text { Name } \\ \text { OPENRQ }\end{array} & \begin{array}{l}\text { Bytes } \\ \text { DSECT }\end{array} \\ \text { DPARM } & 0-7 & \text { Parameter area for error messages } \\ \text { RETMREA } & 8-11 & \begin{array}{c}\text { Address of area to return to after call- } \\ \text { ing subroutine to check user's authority }\end{array} \\ \text { DPLISTA } & 12-95 & \text { Parameter list passed to AUIHCHK } \\ \text { DADRAT } & 12-15 & \text { Address of User's Authority Item } \\ \text { DADREC } & 16-19 & \text { Address of record to be checked } \\ \text { DFNLFN } & 20-21 & \text { Length of file name }\end{array}$


Name

DFIINAM

DFDADEX

DFDADDR

DFDIENN

DSERRER

DINCIL

DNADDR

DNAKIB

OPRQMPAR

OPSAVE

ATMODEAR

TMODEAR

USERID

FCBFIF

FIFKYARG

PAROPEN

AFCBFIF

FILEFIB
Bytes

$22-75$

76

$77-79$

$80-83$

84

85

$86-87$

$88-91$

$92-95$

$96-99$

$100-103$

$104-107$

108 - 111

$112-183$

$184-187$

$188-189$

$190-219$

$220-227$

$228-739$

$740-747$

$748-759$

$748-751$

$752-755$
Content

File name of file whose access is to be checked

Code for presence of partitioning description

Address of partitioning logical expreseion

Length of partitioning logical expression

Code for service request

Code for checking level

Control information about limiting description

Address of internal form of limiting description

Address of Key Information Buffer (KIB) for limiting description

Parameters for \$REQM

Save area for OPNPROC

Address of area for TMODE macro

Length of area for TMODE macro

Area for TMODE macro

User Identification

Area for File Control Block (FCBO of File of Files (FIF)

Parameter in FCB of FIB

Parameter list passed to FIFDIRSI

Address of FCB of FIF

Address of File Information Block for requested file 


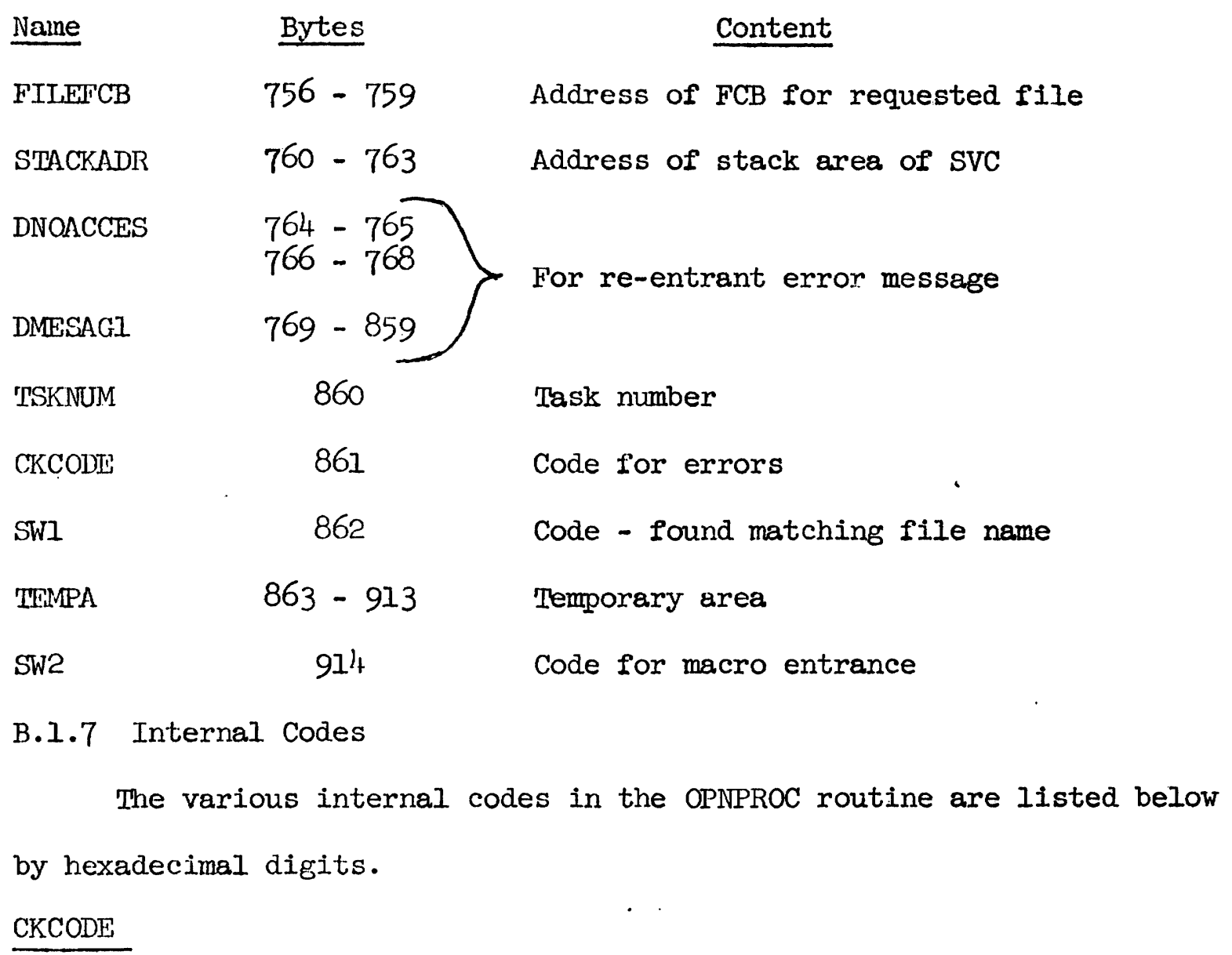

Return from AUTHCHK

$\begin{array}{ll}X^{\prime} 00^{\prime} & \text { Access granted } \\ X^{\prime} O I^{\prime} & \text { Access denied }\end{array}$

Return from SSBACQR

$\begin{array}{ll}X^{\prime} 00^{\prime} & \text { SSB exists but has not been acquired } \\ X^{\prime} 04^{\prime} & \text { SSB exists and has been acquired } \\ X^{\prime} 08^{\prime} & \text { SSB does not exist }\end{array}$

Return from SSBGTNU

$$
X^{\prime} 10^{\prime} \quad \text { RTQM error }
$$

Return from SSBIOGON

$X^{\prime} 00^{\prime}$ SSB exists but has not been acquired 
DESCODE (Description code)

$\mathrm{X}^{\prime} 00^{\prime}$

$X^{\prime} F^{\prime}{ }^{\prime}$

DFDADEX

$$
X^{\prime} F^{\prime} F^{\prime}
$$

FSBCTRI

$X^{\prime} F^{\prime} F^{\prime}$

FUNCODE (Function code)

$x^{\prime} 42^{\prime}$

$x^{\prime} 43^{\prime}$

$\underline{\mathrm{SSBCL}}$

$x^{\prime} 42^{\prime}$

$x^{143}$

SSBCI+1

$$
\begin{array}{ll}
X^{\prime} 00^{\prime} & \text { File partition EDMF closed } \\
X^{\prime} F^{\prime} & \text { File partition EDMF open }
\end{array}
$$

SSBCTL6

$\mathrm{X}^{\prime} \mathrm{F}^{\prime} \mathrm{F}^{\prime}$

SSBDTBIN

$X^{\prime} F^{\prime} F^{\prime}$

SWI

$$
\mathrm{X}^{\prime} \mathrm{FF}^{\prime}
$$

Code that indicates matching file name found on SSB

SW2
Partitioning logical expression not present

Partitioning logical expression present

Code that indicates partitioning logical expression present

Code that indicates good pointer in FSB block

Read type open

Update type open

Read type open

Update type open

Code that indicates good pointer in SSB block

Code that indicates user description block present 


\section{B.1.8 Return Codes}

All return codes can be found in the right-most byte of Register 15 and they are listed below by hexadecimal digits.

$$
\begin{array}{ll}
\mathrm{X}^{\prime} \mathrm{OO} & \text { Everything O.K. } \\
\text { Otherwise } & \text { Error occurred }
\end{array}
$$

\section{B.1.9 Flowchart}

Figures B.1.a - B.I.d contain the flowchart for the OPNPROC routine. 


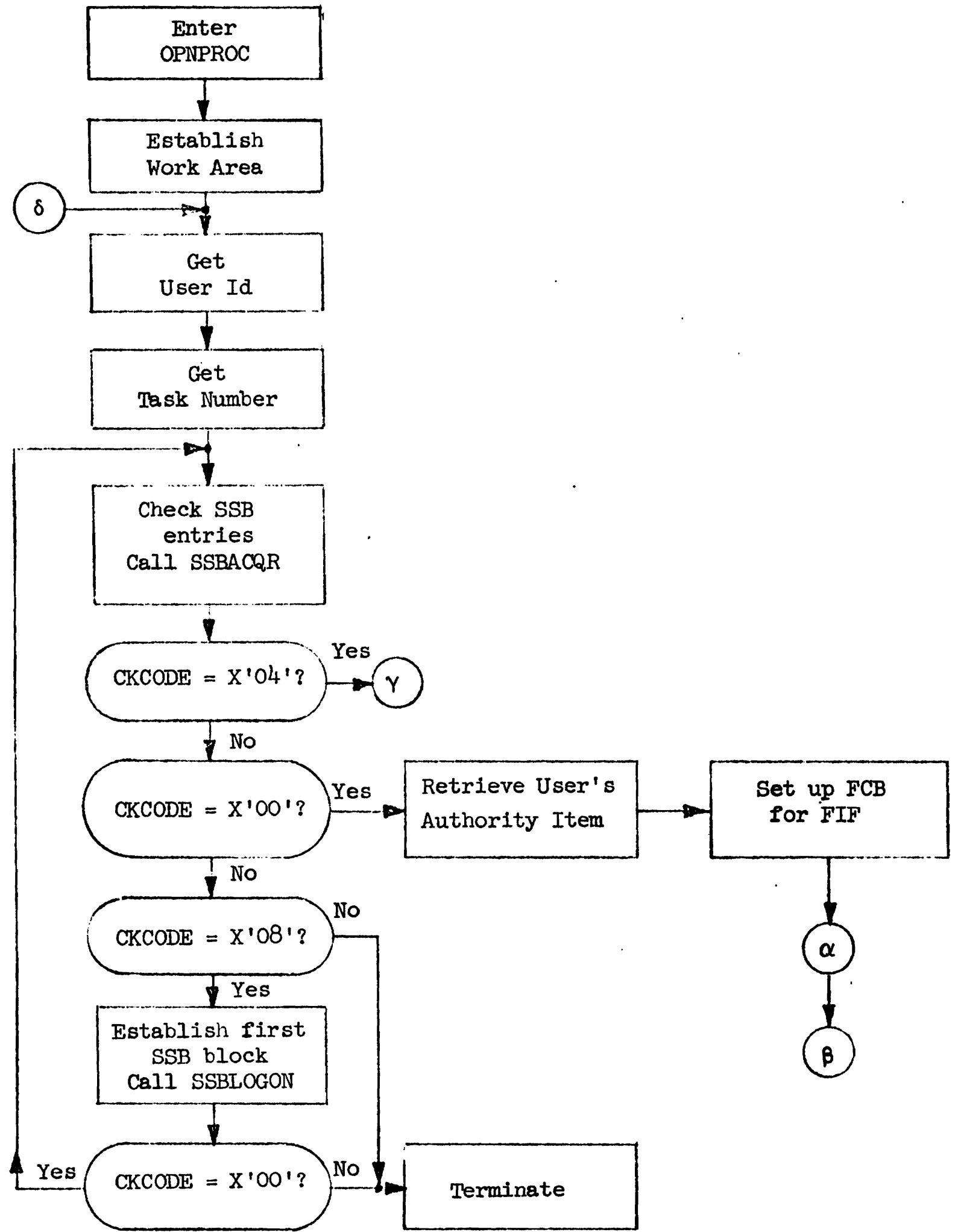

Figure B.1.a: OPNPROC Initialization. Retrieve SSB Chain. 


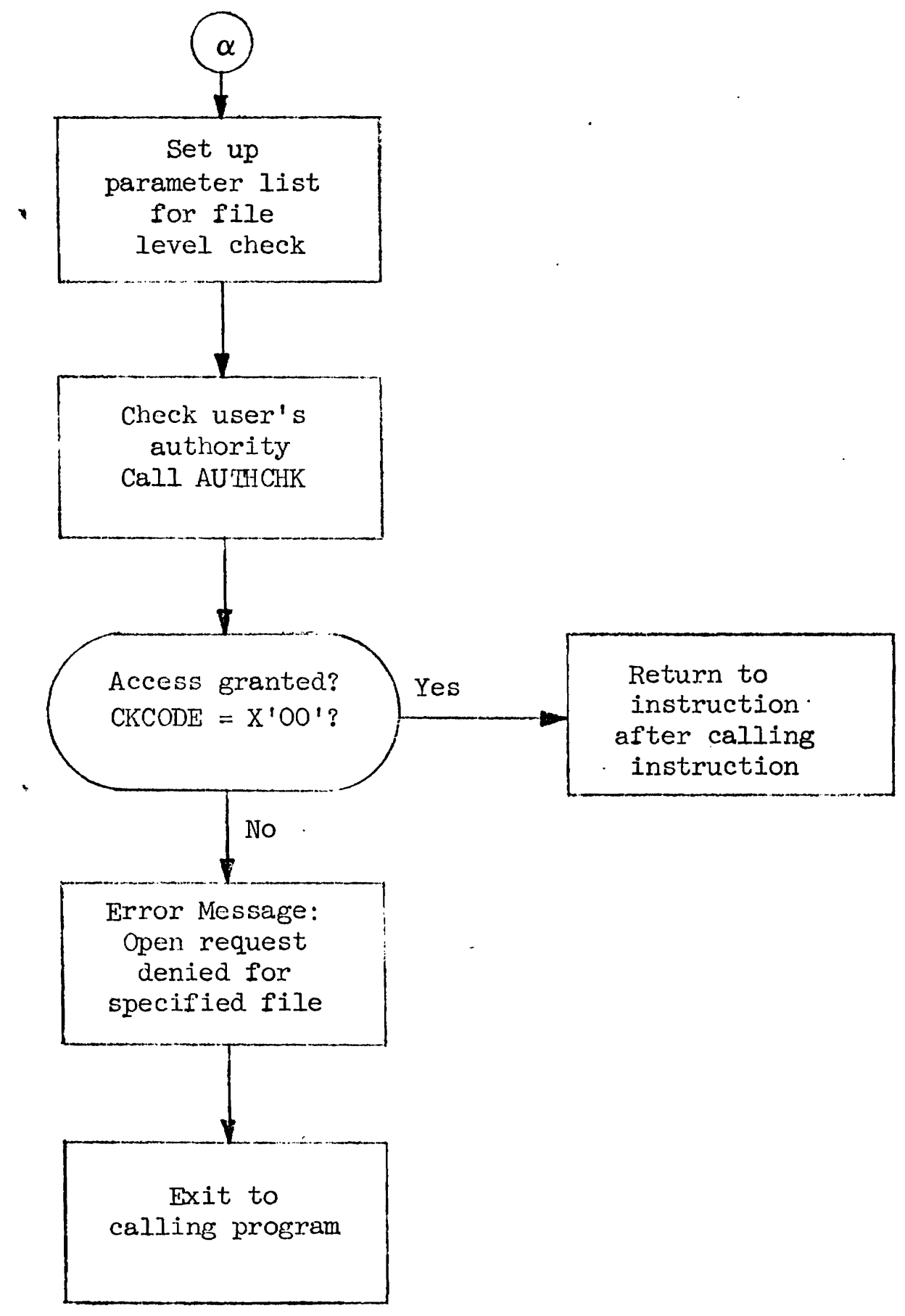

Figure B.1.b: Authority Item Check 


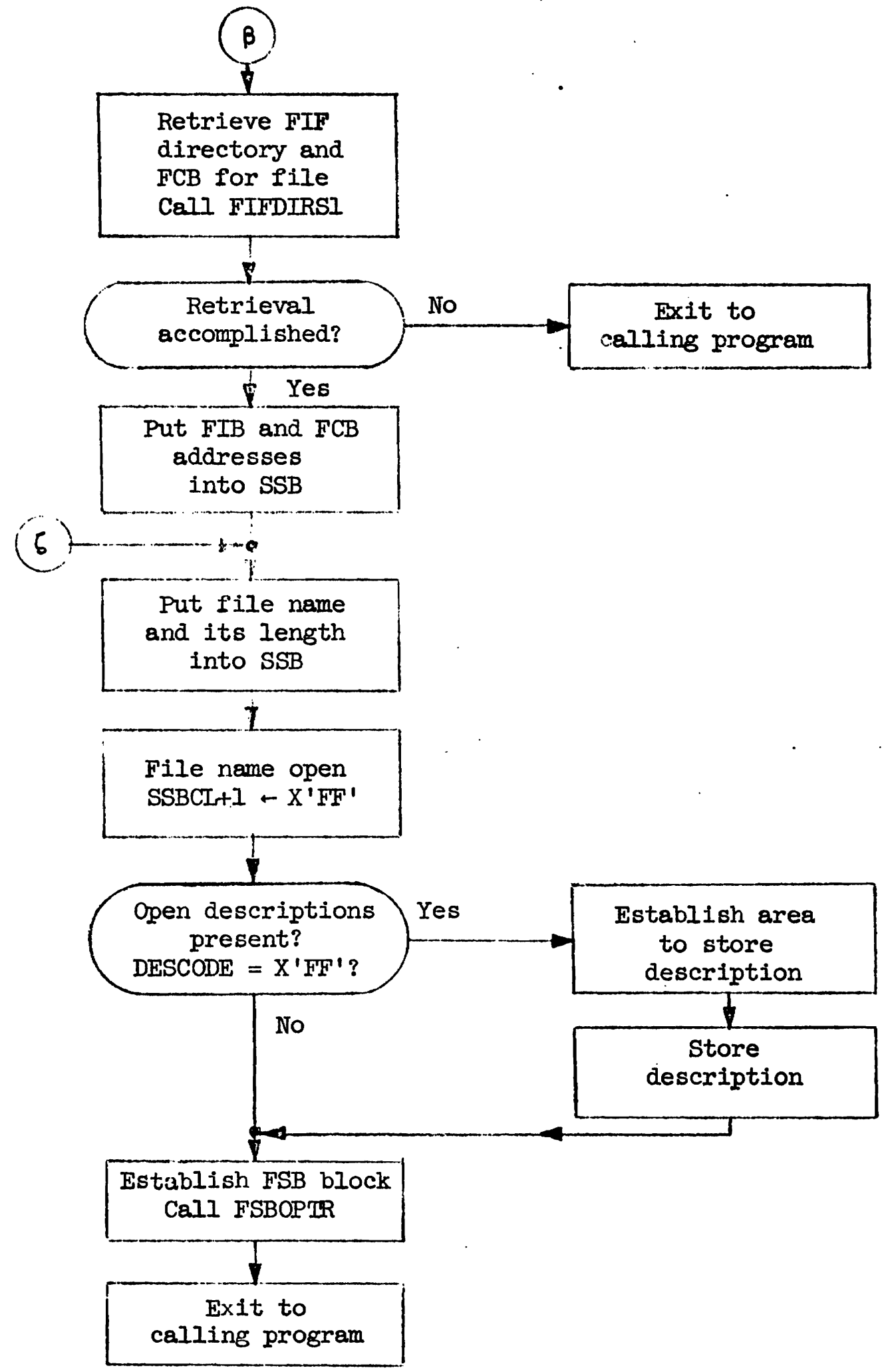

Figure B.I.c: Set up SSB. Establish FSB. 


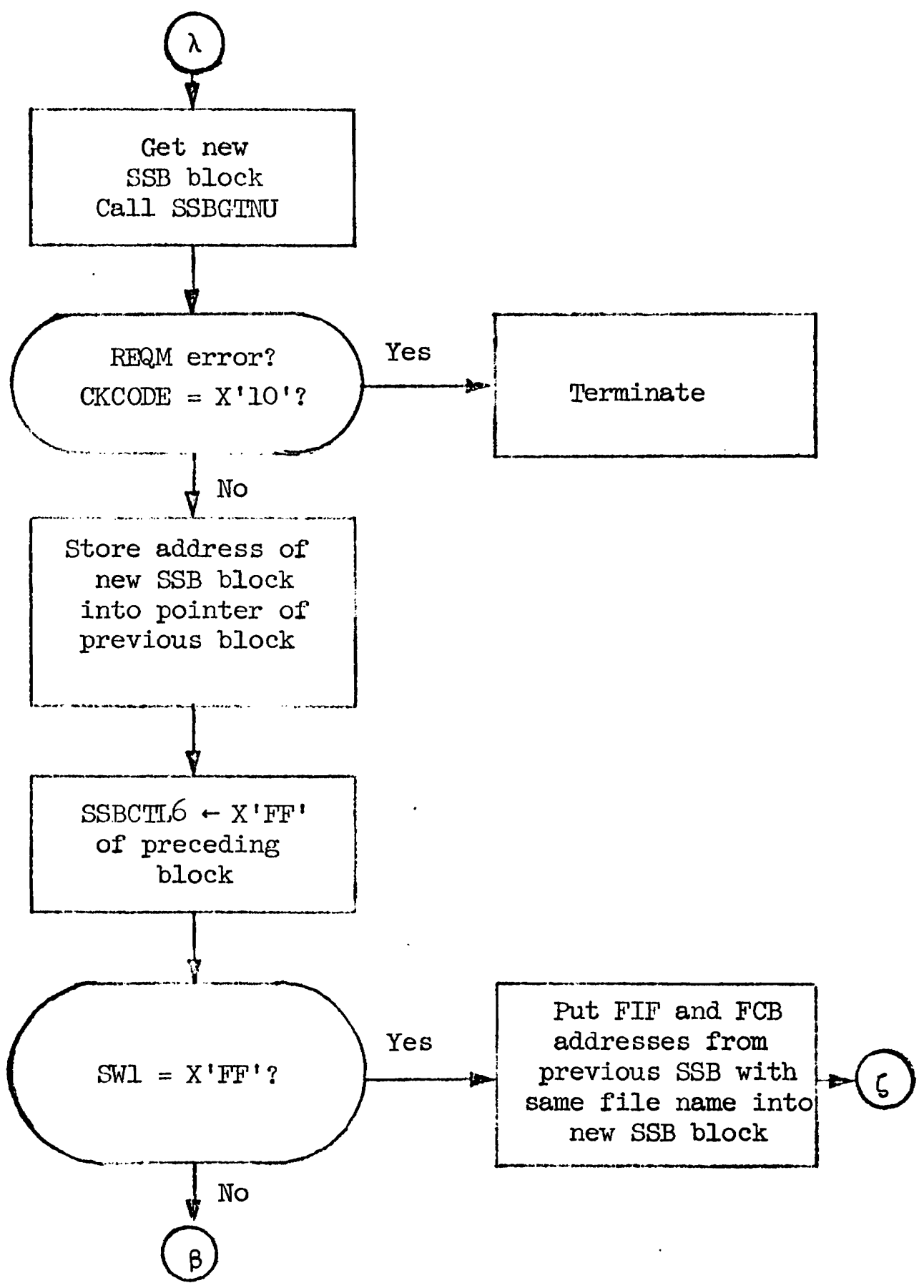

Figure B.1.d: Get New SSB 


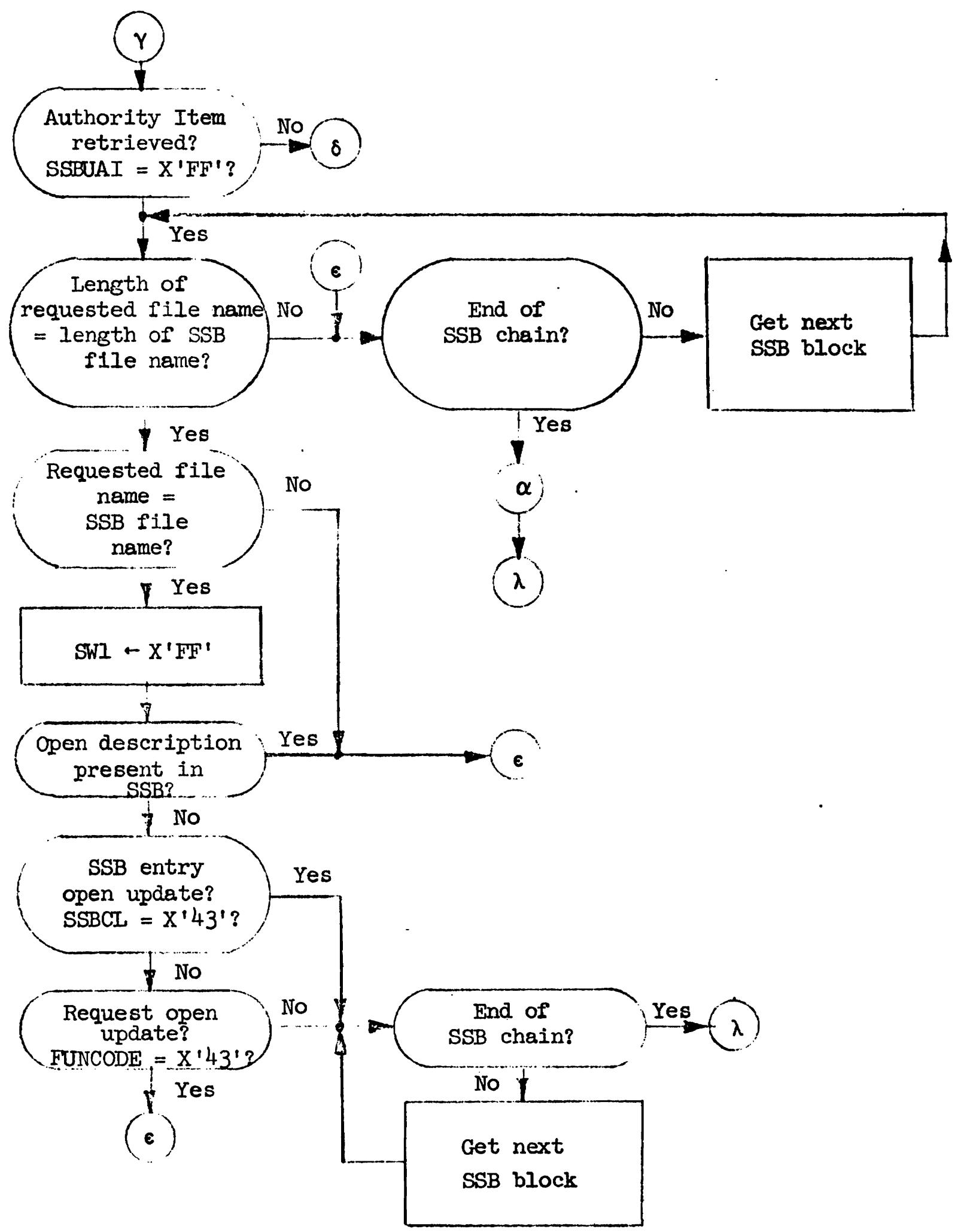

Figure B.1.e: SSB Check 


\section{B.2 Routine FIFDIRSI}

The FIFDIRSI routine is the second of two routines that implement the EDMF's Open Function. This routine establishes the File Control Block (FCB) for the File of Files (FIF), searches the FIF directory and retrieves whe File Information Block (FIB) for the requested file. B.2.1 Entry Points

FIFDIRSI is the only entry point in this routine.

\section{B.2.2 Exit Points}

FIFDIRS has two exit points. The normal exit begins at ouT2 and the error exit begins at aTI. In both cases, program control is returned to the calling program.

\section{B.2.3 Fxternal Subroutine Calls}

RETRREC $[6]$ is the only external subroutine called by FIFDIRS. The first time RETRREC is called it retrieves the FIF directory; the second time, it retrieves the FIB for the requested files. The DSECT's that are associated with the FIF directory and the FIB are the following:

Name Bytes Content

DIRFIF $\quad$ DSECT

HFADERD $0-14 \quad$ Header

LENGTHD $\quad 0-2$ Length of FIF directory

CONNID $\quad 3-4 \quad$ Count of FIF directory

IKEYD $\quad 5-9 \quad$ Lowest key in directory

HKEYD $\quad 10-14 \quad$ Highest key in directory

ENTRIES -.. Individual entries

FIB DSECT

$$
0-92 \quad \text { Beginning of FIB }
$$


Name

FCB

RF'B
Bytes

$93-252$

$253-$

Content

File Control Block

Record Control Block

B.2.4 Input Parameter Lists

There are two necessary input parameter lists for the FIFDIRSI routine. The address of the PAROPEN input list must be in Register I while the address of the PARAMOP input list must be in Register 12. Register 13 must contain the address of the calling routine's save area.

Name

PAROPEN

AFCBFIF

FIIEFIB

FILEFCB

PARAMOP

FTNMLN

FLNAME

FUNCODE

LOGEXPAD

LNLOGEXP

LOGEXP

DESCODE
Bytes

DSECT

$$
0-3
$$$$
4-7
$$

$8-11$

DSECT

$0-1$

$2-55$

56

$57-59$

$60-63$

$64-190$

191
Content

Address of FCB of FIF

Address of FIB of the requested file

Address of FCB of the requested file

Length of file name

File name (left justified with spaces)

Code for type of open requested

Address of partitioning logical expression

Length of partitioning logical expression

Partitioning logical expression if included in OPN macro

Code for presence of partitioning description 
B.2.5 Register Conventions

The registers in FIFDIRSI are assigned in the following manner:

$\underline{\text { Register }}$

0

1

2

3

4

5

6

7

8

9

10

11

12

13

14

15

\section{Utilization}

Not used

Address of parameter list given to called subroutine

Length of FIF directory

Length of requested file name

Address and base of DIRFIF. Address and base of FIB.

Base for FIFDIRSI

Length-I of file name in FIF directory. Miscellaneous use.

Pointer to entry in FIF directory

$=\mathrm{H}^{\prime} 7^{\prime}$

Address of last byte in FIF directory

Address and base of input parameter list (PAROPEN)

Address and base of FIFDIRSI work area

Address and base of input parameter list (PARAMOP)

Address of FIFDIRSI save area

Return address in FIFDIRSI

Subroutine call address. Error codes.

\section{B.2.6 Internal Work Area}

The internal work area (SUPI) used by the FIFDIRSI routine also contains the parameter list (PLIST) to be passed to RETRREC [6 ]. The - work area has the following format: 


\begin{tabular}{lcl} 
Name & Bytes & \multicolumn{1}{c}{ Content } \\
SUPI & DSECT & \\
SAVEI & $0-71$ & Save area for FIFDIRSI \\
WKAREA & $72-75$ & Temporary work area \\
OPPARAM & $76-83$ & Parameter area for DMS open \\
CLPARAM & $84-91$ & Parameter area for DMS close \\
WFCB & $92-603$ & File Control Block \\
KEYARG & $604-611$ & Parameter in FCB \\
PLIST & $612-627$ & Parameter area passed to REIRREC \\
PFCBADDR & $612-615$ & Address of FCB \\
PRECADDR & $616-619$ & Address of area to place retrieved record \\
PISAM & $620-624$ & ISAM key for requested record \\
PLREC & $625-627$ & Length of area to place retrieved record \\
B.2.7 Internal Codes &
\end{tabular}

$X^{\prime} O O^{\prime}$
$X^{\prime} F^{\prime}$

Partitioning logical expression not present Partitioning logical expression present

FUNCODE (Function code)

$$
\begin{array}{ll}
X^{\prime 42} 42^{\prime} & \text { Read type open } \\
X^{\prime} 43^{\prime} & \text { Update type open }
\end{array}
$$

\section{B.2.8 Return Codes}

All return codes can be found in the right-most byte of Register 15 and they are listed below by hexadecimal digits.

$$
\begin{array}{ll}
X^{\prime} 00^{\prime} & \text { Everything } 0 . K . \\
X^{\prime} 04^{\prime} & \text { Unable to open FIF }
\end{array}
$$


$B-18$

$\begin{array}{ll}X^{\prime} 08^{\prime} & \begin{array}{l}\text { Unable to retrieve FIF or FIB of } \\ \text { requested file }\end{array} \\ X^{\prime O C '} & \begin{array}{l}\text { Requested file does not exist in the } \\ \text { system }\end{array} \\ X^{\prime O F '} & \text { REQM error }\end{array}$

B.2.9 Flowchart

Figures B.2.a - B.2.b contain the flowchart for the FIFDIRSI routine. 


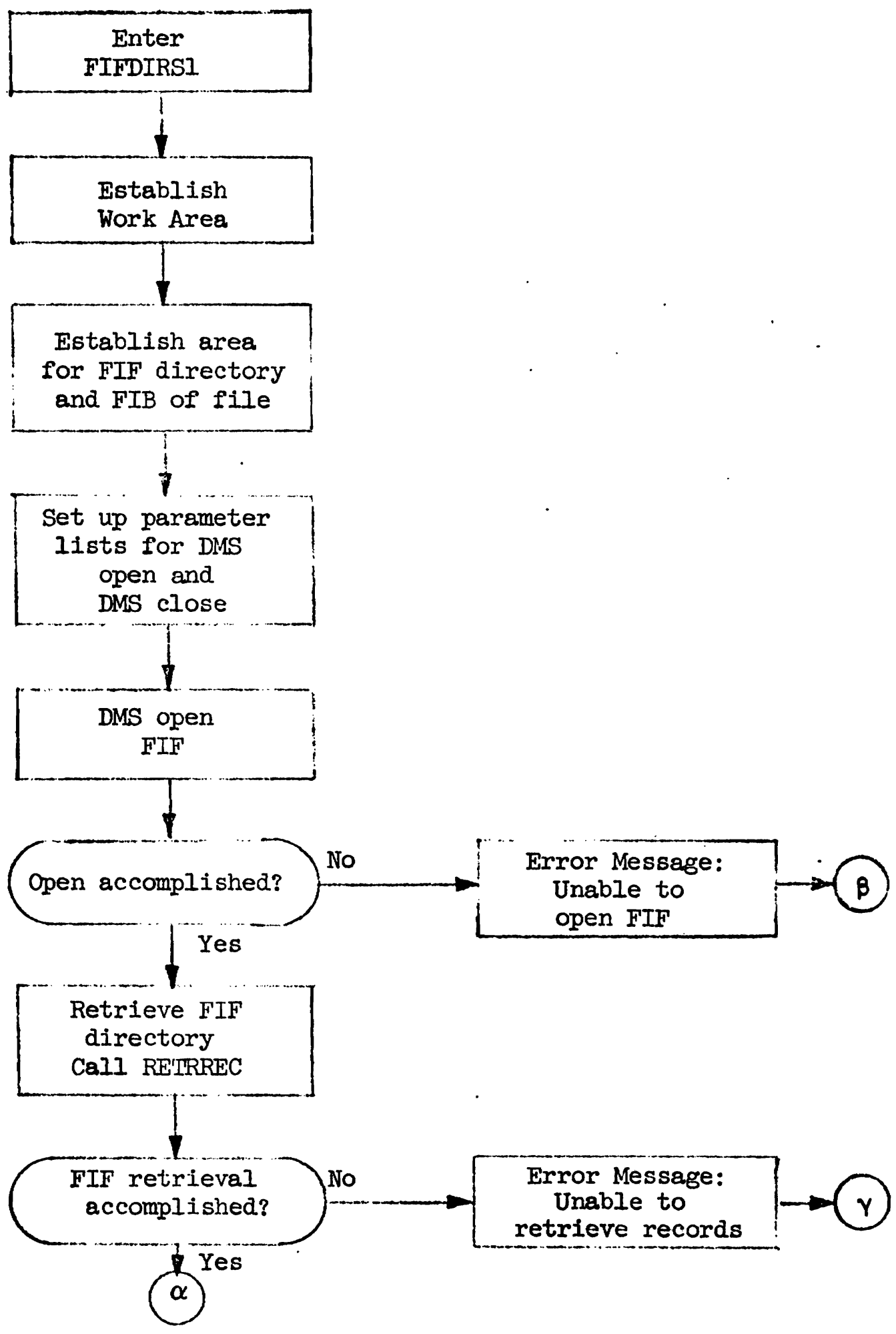

Figure B.2.a: FIFDIRSI Initialization and FIF Directory Retrieval 


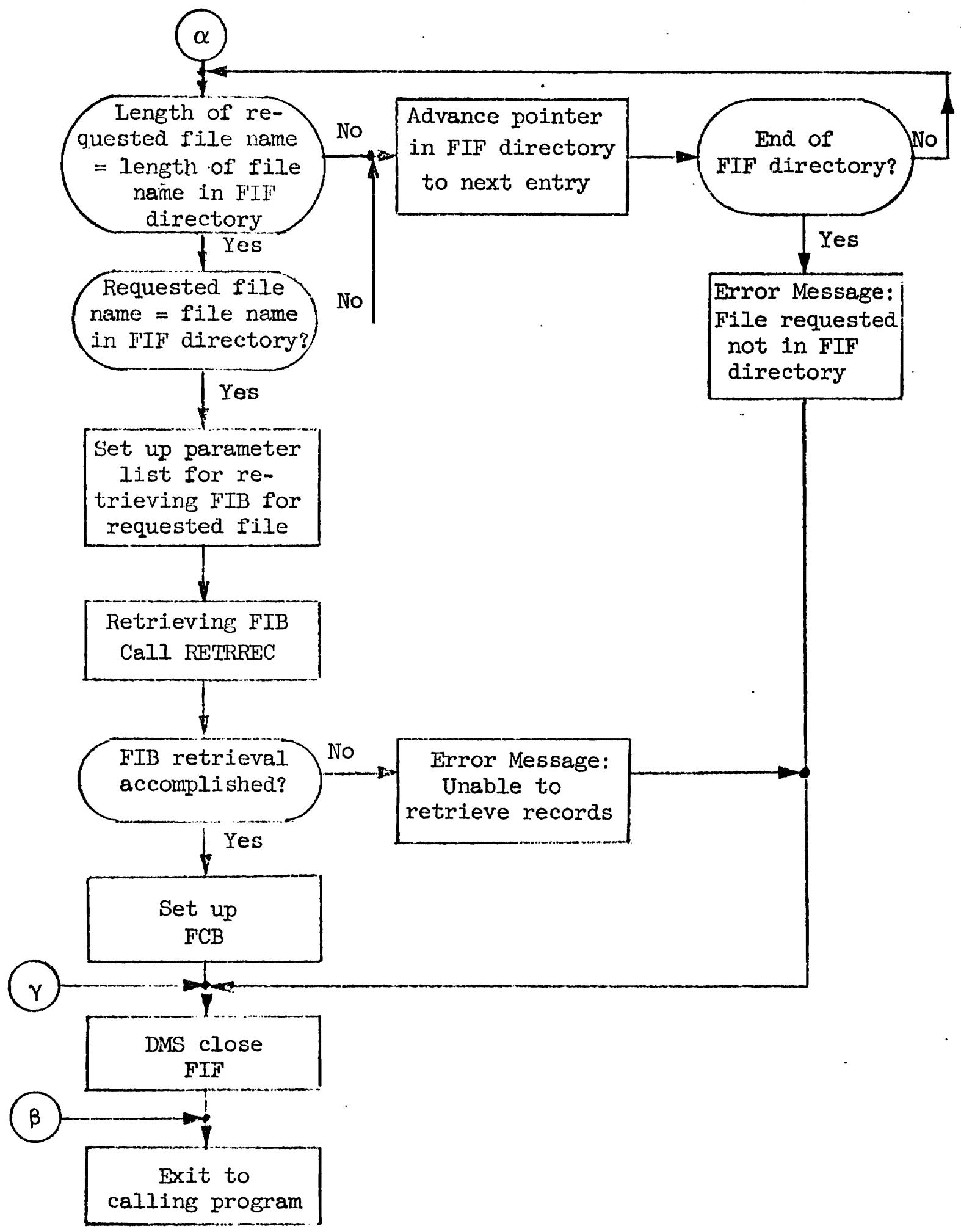

Figure B.2.b: Retrieval of File FIB and FCB 


\section{B.3 Routine MACPROC}

The MACPROC routine obtains necessary information before the retrieval optimization phase is entered. The main function of this routine is to check if an EDMF open has been issued and if so, issue a DMS open. Also, if entry is non-conversational in nature, the routine obtains the internal form of the user's request description. If entry is conversational, the internal form has already been obtained.

\section{B.3.1 Entry Points}

There are three entry points. MACPROC is used when entry is from a user program (non-conversational); COMENTER is the point a conversational user enters. After the EDMF has processed a retrieval, it is necessary to DMS close the specified file. This is accomplished at the CLFIIE entry point.

\section{B.3.2 Exit Points}

MACPROC has two exit points. One is the normal exit point and the other is used when an error occurs. The normal exit is to the REIRIEVE routine. The error exit is at CHKEXIT.

\section{B.3.3 External Subroutine Calls}

Two external subroutines are called by MACPROC. The first is to the location ESQCAT to obtain the task number. The second is to the entry point SSBACQR of the SSBOPTR routine [9]. This is used to obtain the SSB chain for a specific user. The DSECT that is associated with the SSBOPTR routine is the following:

$\begin{array}{lcl}\text { Name } & \text { Bytes } & \text { Content } \\ \text { SSB } & \text { DSECT } \\ \text { SSBHDR } & 0-7 & \text { SSB Header } \\ \text { SSBUAI } & 0-3 & \text { Address of User's Authority Item }\end{array}$




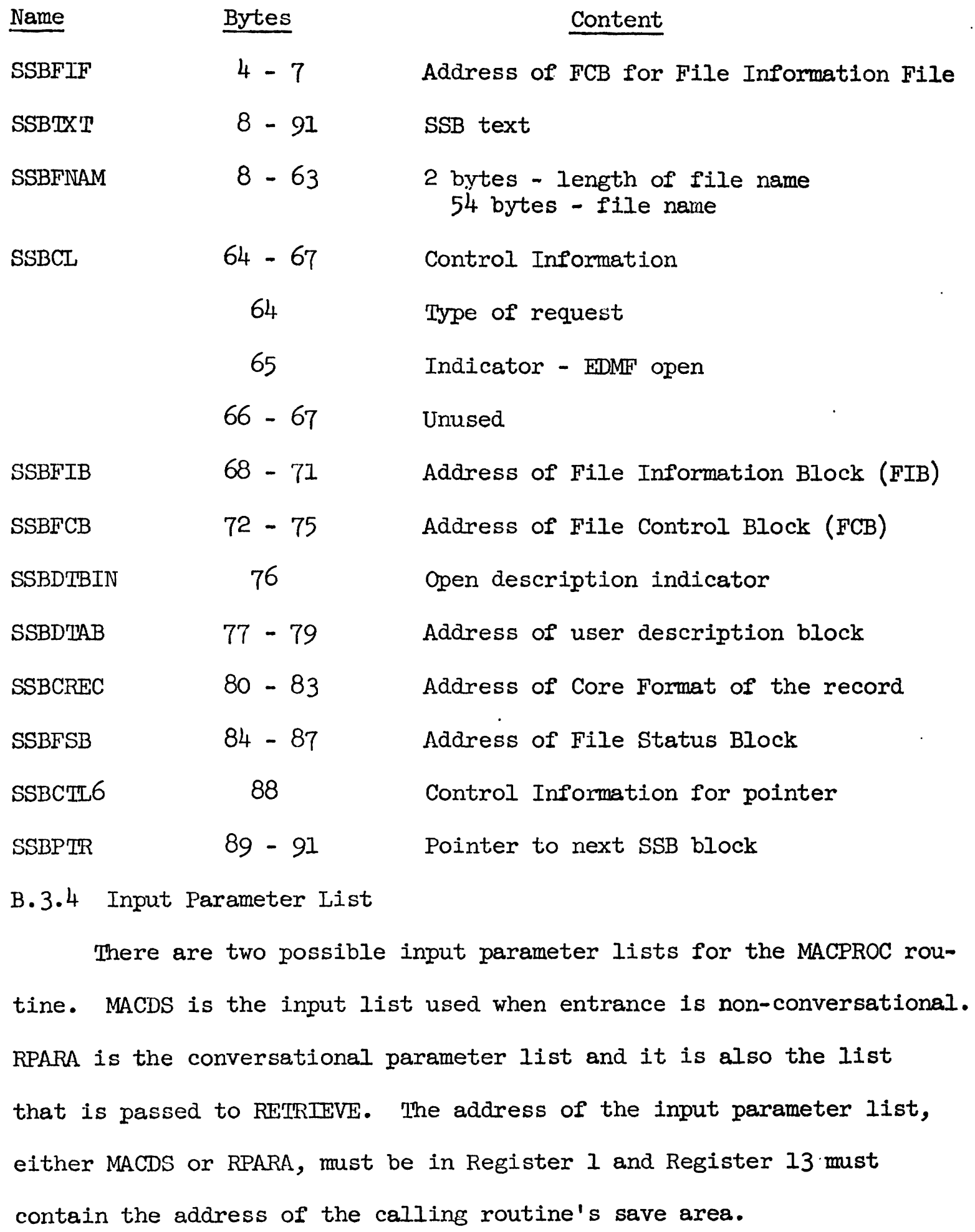




\begin{tabular}{|c|c|c|}
\hline Name & Bytes & Content \\
\hline RPARA & DSECT & \\
\hline $\mathrm{AFCB}$ & $0-3$ & Address of File Control Block (FCB) \\
\hline RF'BA & $4-7$ & Address of Record Format Block (RF'B) \\
\hline USRID & $8-15$ & User Identification \\
\hline RECNO & $16-17$ & $\begin{array}{l}\text { Number of requested records to be } \\
\text { retrieved. }\end{array}$ \\
\hline OUTSPEC & $18-27$ & Output specification \\
\hline FLNMLN & $28-29$ & Length of file name \\
\hline FTNAME & $30-83$ & File name (left justified with spaces) \\
\hline FUNCODE & 84 & Code for function requested \\
\hline CONTROI & 85 & $\begin{array}{l}\text { Part of internal form of user's descrip- } \\
\text { tion }\end{array}$ \\
\hline LIIEP & $86-87$ & Length of $\mathrm{DCB}$ and $\mathrm{KIB}$ \\
\hline IDCB & $88-89$ & Length of $\mathrm{DCB}$ \\
\hline $\mathrm{DCB}$ & -- & Actual Description Control Block (DCB) \\
\hline KIB & -- & Actual Key Information Buffer (KIB) \\
\hline MACDS & DSECT & \\
\hline MRECNO & $0-1$ & $\begin{array}{l}\text { Number of requested records to be } \\
\text { retrieved }\end{array}$ \\
\hline MOUTSPEC & $2-11$ & Output specification \\
\hline MFLNMLEN & $12-13$ & Length of file name \\
\hline MFLEIIAME & $14-67$ & File name (left justified with spaces) \\
\hline MFUNCODE & 68 & Code for function requested \\
\hline MADLOGEP & $69-71$ & Adaress of logical expression \\
\hline MIENLEXP & $72-75$ & Length of logical expression \\
\hline MILOGEXP & $76-202$ & $\begin{array}{l}\text { Logical expression if included in the } \\
\text { macro.- }\end{array}$ \\
\hline
\end{tabular}




\section{B.3.5 Register Conventions}

The registers in MACPROC are assigned in the following manner:

\begin{tabular}{|c|c|}
\hline Register & Utilization \\
\hline 0 & Not used \\
\hline 1 & $\begin{array}{l}\text { Address of parameter list given to called } \\
\text { subroutine. Miscellaneous use. }\end{array}$ \\
\hline 2 & Miscellaneous use \\
\hline 3 & Base for MACPROC \\
\hline 4 & Miscellaneous use \\
\hline 5 & Not used \\
\hline 6 & $\begin{array}{l}\text { Counter for number of characters in User } \\
\text { Id. Miscellaneous use. }\end{array}$ \\
\hline 7 & Not used \\
\hline 8 & $\begin{array}{l}\text { Length of requested file name. Miscellaneous } \\
\text { use. }\end{array}$ \\
\hline 9 & Address and base of SSBTEXT. \\
\hline 10 & Address and base of MACPROC work area. \\
\hline 11 & $\begin{array}{l}\text { Address and base of input parameter list } \\
\text { (MACDS) }\end{array}$ \\
\hline 12 & $\begin{array}{l}\text { Address and base of input parameter list } \\
\text { (RPARA) }\end{array}$ \\
\hline 13 & Address of MACPROC save area \\
\hline 14 & Return address in MACPROC \\
\hline 15 & Subroutine call address. Error codes. \\
\hline
\end{tabular}

B.3.6 Internal Work Area

WORK is the name of the internal work area used by MACPROC and it has the following format: 


\begin{tabular}{|c|c|c|}
\hline Name & Bytes & Content \\
\hline WORK & DSECT & \\
\hline SAVE2 & $0-71$ & Save area for MACPROC \\
\hline DPRM & $72-79$ & Parameter area for error messages \\
\hline OPPARM & $80-87$ & Parameter area for DMS open \\
\hline CL.PARAM & $88-95$ & Parameter area for DMS close \\
\hline RATMODE & $96-99$ & Address of area for TMODE macro \\
\hline & $100-101$ & Length of area for TMODE macro \\
\hline RTMODE & $102-131$ & Area for TMODE macro \\
\hline ADSTACK & $132-135$ & Address of stack of SVC \\
\hline TEMPF & $136-139$ & Temporary area \\
\hline TEEMPH & $140-141$ & Temporary area \\
\hline CHKCODE & 140 & Code for errors in SSB routine \\
\hline TASKNUM & 141 & Task number \\
\hline DNTMOPN & $\begin{array}{l}142-143 \\
144-146\end{array}$ & \\
\hline DMI & $147-242$ & ror message \\
\hline DNOPDMS & $\begin{array}{l}243-244 \\
245-247\end{array}$ & \\
\hline DM2 & $248-317$ & \\
\hline TEMMP & $318-368$ & Temporary area \\
\hline SWI & 369 & Code for macro entrance \\
\hline B. 3.7 & 1 Codes & \\
\hline
\end{tabular}

The various internal codes in the MACPROC routine are listed below by hexadecimal digits. CHKCODE 
FUNCODE (Function code)

$$
x^{\prime} 22^{\prime}
$$

MFUNCODE (Function code)

$$
X^{\prime} 22^{\prime}
$$

SSBCI+1

$$
\begin{array}{ll}
\mathrm{X}^{\prime} \mathrm{OO} \mathrm{O}^{\prime} & \text { File EDMF closed } \\
\mathrm{X}^{\prime} \mathrm{FF}^{\prime} & \text { File EDMF open }
\end{array}
$$

SSBCTL6

$$
\mathrm{X}^{\prime} \mathrm{FF}^{\prime}
$$

$\mathrm{X}^{\prime} \mathrm{FF}^{\prime}$

SSBDTBIN

$$
\mathrm{X}^{\prime} \mathrm{FF}^{\prime}
$$
block

SWI
Code for retrieval

Code for retrieval

Code that indicates good pointer in SSB

\section{Code that indicates user description block present}

B.3.8 Flowchart

Figures B.3.a - B.3.C contain the flowchart for the MACPROC routine. 


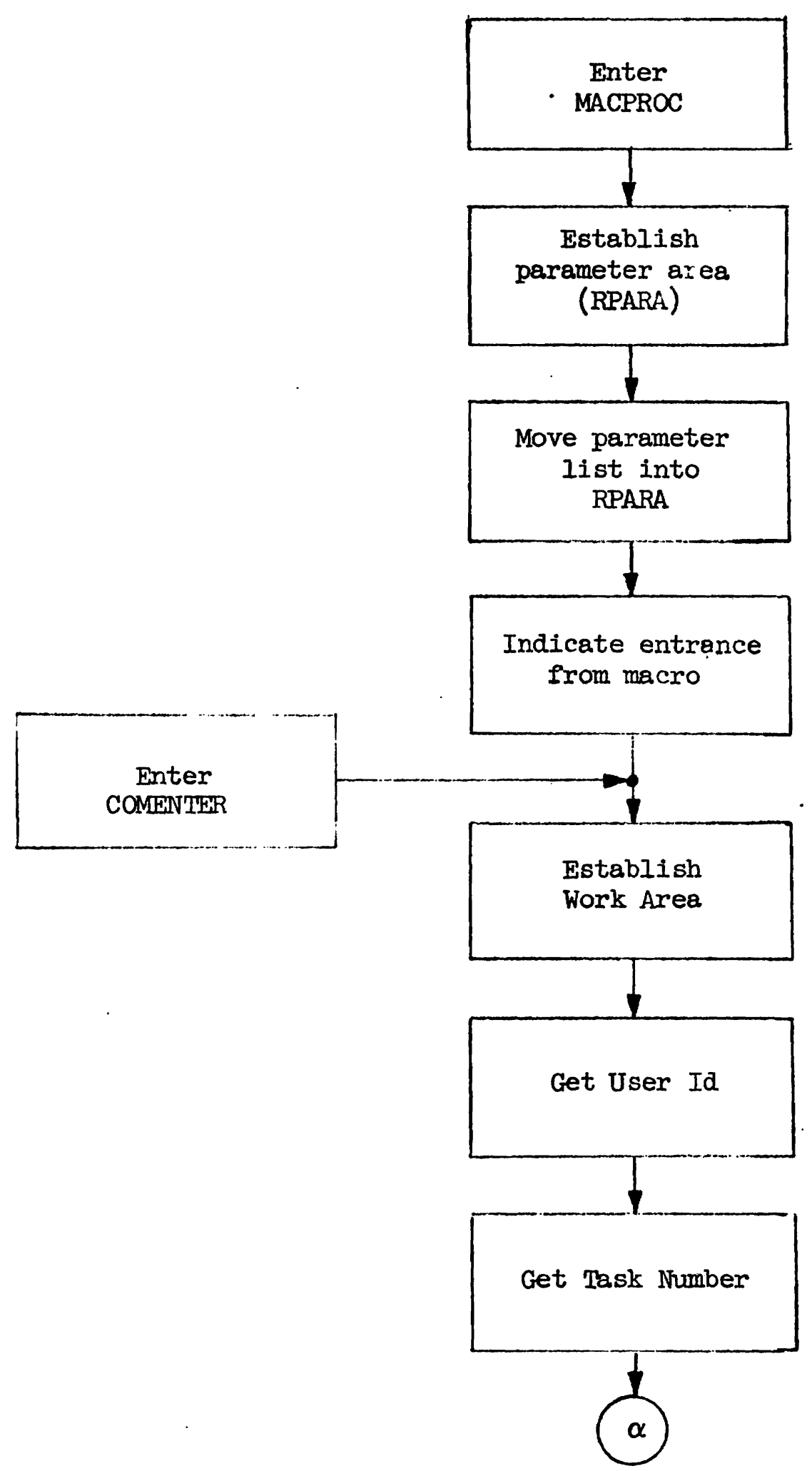

Figure B.3.a: MACPROC Initialization 


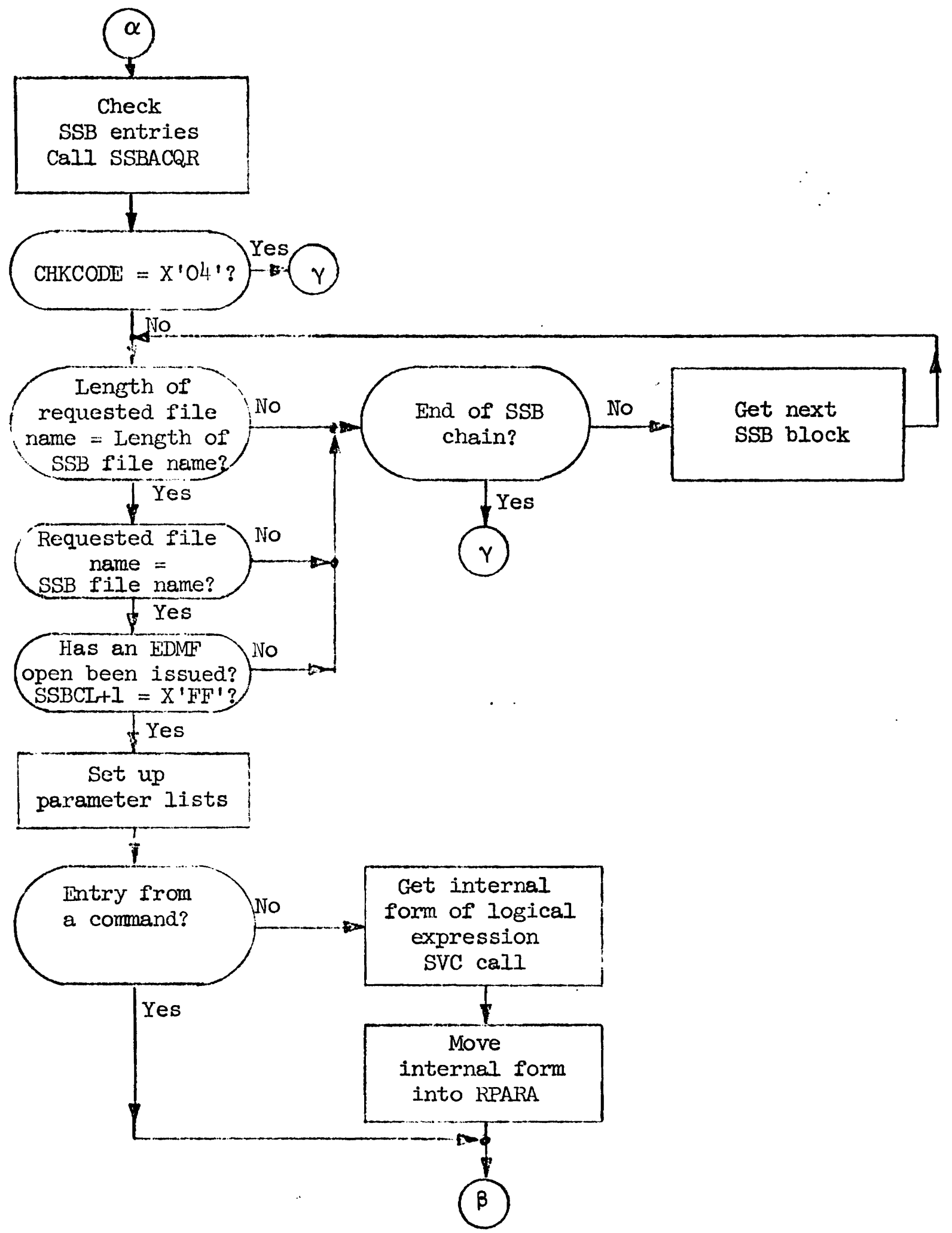

Figure B.3.b: SSB Check and Translation of Logical Expression 


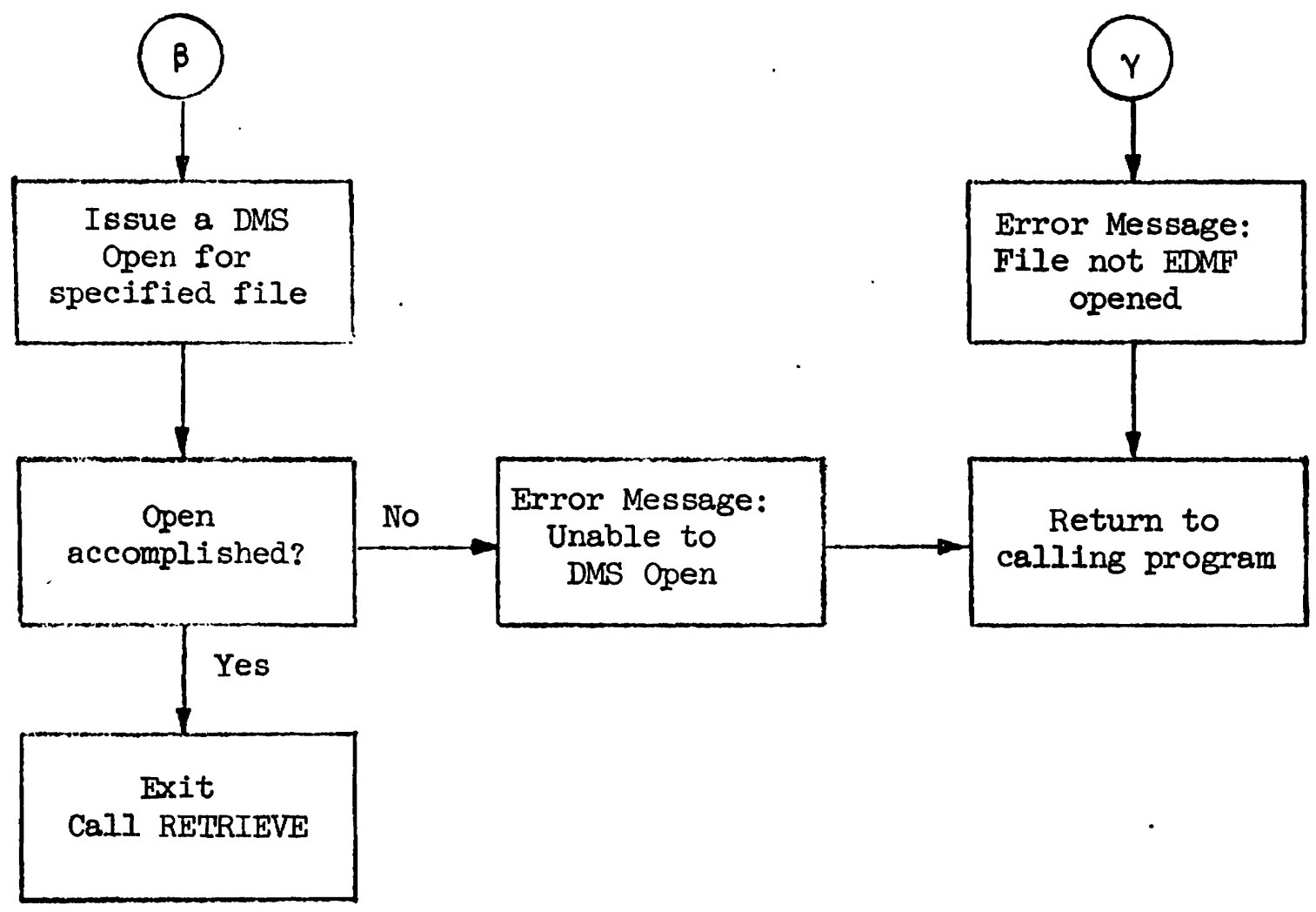

Figure B.3.c: DMS Open 


\section{B.4 Routine RETRIEVE}

The routine RETRIEVE is the part of the supervisor that implements the Retrieval Optimization function by selecting the prime keywords and also obtaining the ISAM keys that are Head of Iist Addresses.

\section{B.4.1 Entry Points}

There are two entry points. The normal entrance is at RETRIEVE. The second entrance is at SPCENOI; this is an error message entrance for other routines that cannot request memory. B.4.2 Exit Points

RETRIEVE has three exit points. One is the normal exit point and the other two are used when an error occurs. The normal exit point begins at MARK and a call for the routine FORPROG is issued. The error exits are at ROUT and ROUT2.

B.4.3 External Subroutine Calls

Two external subroutines are called by RETRIEVE. The first is RETRDIR which retrieves the requested file's highest level directory. The second subroutine called is DECODE [3]. DECODE is used to decode the directory to determine the prime keywords and it also passes the corresponding HOTA's to RETRIEVE.

B.4.4 Input Parameter List

The address of the input parameter list (RPARA) must be in Register 1 and Register 13 mast contain the address of the calling routine's save area. 


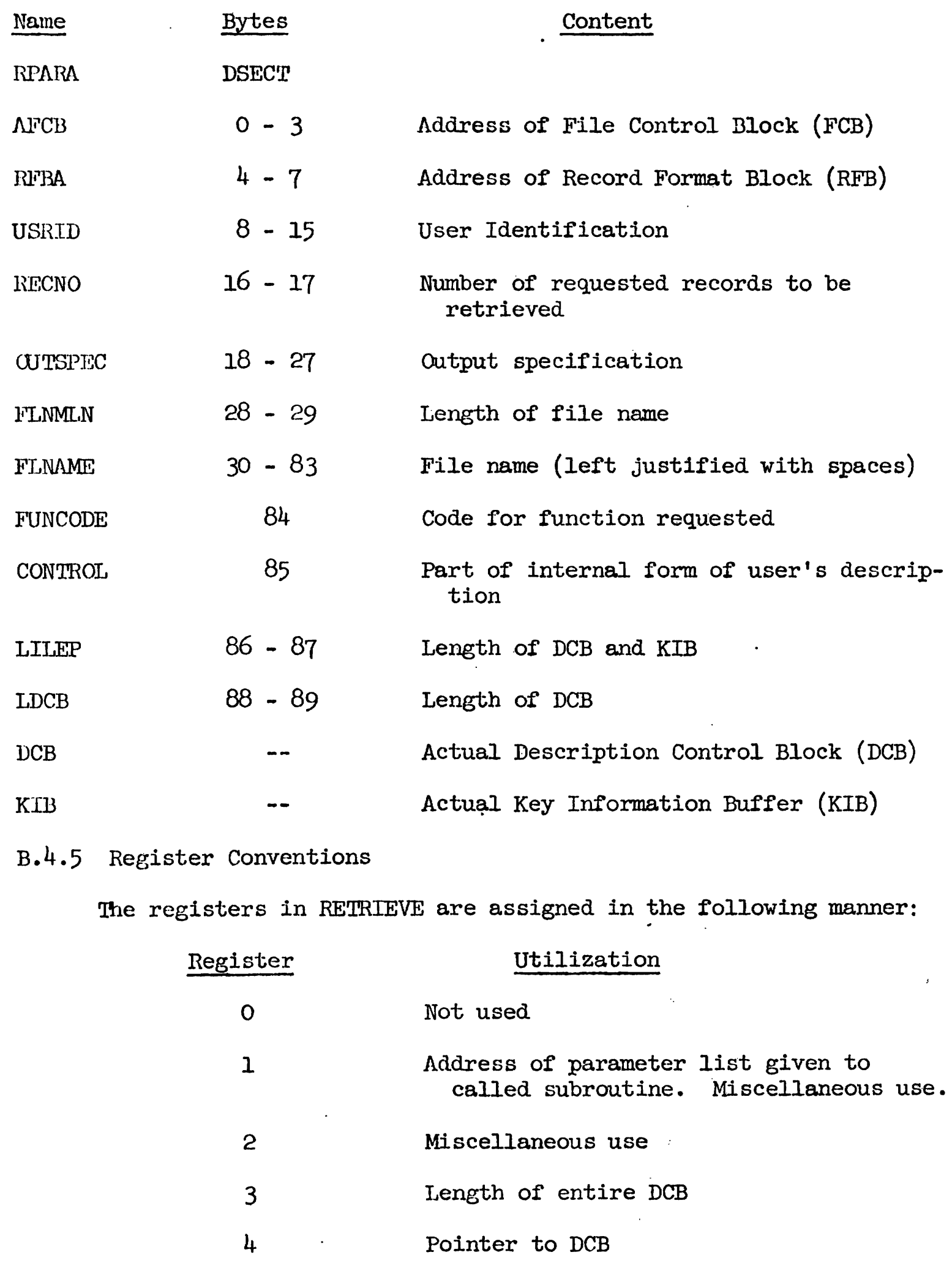




\begin{tabular}{cl} 
Register & \multicolumn{1}{c}{ Utilization } \\
5 & Base for RETRIEVE \\
6 & Length of DCB segments (18) \\
7 & Not used \\
8 & Pointer to PRIMEKEY stack \\
9 & Address and base of input parameter \\
10 & Iist (RPARA) \\
11 & Pointer to ADDRESS. Miscellaneous use. \\
12 & Address and base of RETRIEVE work area \\
13 & Pointer to RQADD. Miscellaneous use. \\
14 & Address of RETRIEVE save area \\
15 & Return address in RETRIEVE \\
& Subroutine call address. Error codes.
\end{tabular}

B.4.6 Internal Work Area

The internal work area (SUP) used by RETRIEVE also contains the parameter lists for some of the routines called by RETRIEVE. LISTP is passed on to the RETRDIR and the DECODE routine while PLFOR is passed to DECODE and FORPROG. The work area has the following format: Name Bytes Content

SUP DSECT

DNOAT $0-1$

$\left.\begin{array}{ll}2 & -4 \\ \text { DMSGI } & 5-59 \\ \text { DNOVAL } & 60-61 \\ & 62-64 \\ \text { DMSG2 } & 65-132\end{array}\right\}$

For re-entrant error message

$133-135$

Not used

DPARAM 


\begin{tabular}{|c|c|c|}
\hline Name & Bytes & Content \\
\hline NLENG & $144-151$ & \\
\hline $\mathrm{N}$ & $144-147$ & Smallest $n^{*}$ in conjunct \\
\hline LENGTII & $148-151$ & Length of associated HOLA's \\
\hline 'LA\}EA & $152-159$ & \\
\hline AREAN & $152-155$ & $\mathrm{~N}$ of current keyword \\
\hline TIIENGIII & $156-159$ & Length of associated HOLA's \\
\hline ENDPRKY & $160-163$ & Address of PRTMEKKEY - 4 \\
\hline RQADD & $164-327$ & Pointers to ISAM keys, length of keys \\
\hline PRIMEKIYY & $328-491$ & $\begin{array}{l}\text { Pointers to beginning of conjuncts and } \\
\text { prime keywords }\end{array}$ \\
\hline SAVE & $492-635$ & Save area for RETRIEVE \\
\hline REQMPAR & $\begin{array}{l}636-639 \\
640-643 \\
643-647 \\
647-651\end{array}$ & Parameters for $\$ R E Q M$ \\
\hline LISTP & $652-671$ & $\begin{array}{l}\text { Parameter list passed to DECODE and } \\
\text { RETRDIR }\end{array}$ \\
\hline ERRCOIE & 652 & Error code \\
\hline PDCB & $652-655$ & Pointer to current location in DCG \\
\hline PTAREA & $656-659$ & Address of TAREA \\
\hline ISAMILND & 660 & Code for $\mathrm{DECODE}$ \\
\hline PADDRSS & $660-663$ & Address of ADDRESS \\
\hline PISAM & $664-667$ & Pointer to area - where to put ISAM keys \\
\hline PHIDIR & $668-671$ & Address of highest level directory \\
\hline PLFOIR & $672-696$ & Parameter list passed to DECODE and FORPROG \\
\hline $\mathrm{PFCB}$ & $672-675$ & Address of $\mathrm{FCB}$ \\
\hline PKIB & $676-679$ & Pointer to KIB \\
\hline PPRMKY & $680-683$ & Pointer to PRIMEKKEY Stack \\
\hline
\end{tabular}




\begin{tabular}{lcl} 
Name & Bytes & \multicolumn{1}{c}{ Content } \\
RFBADD & $684-687$ & Address of RFB \\
ARQADD & $688-691$ & Pointer to location in RQADD \\
PRECNO & $692-693$ & Number of requested records \\
FNCODE & 694 & Code for function requested \\
ATCKIEEV & 695 & Code for level of Authority Item Check \\
PRCODE & 696 & Code for output
\end{tabular}

$*_{n}=$ number of records in file containing a specified keyword

\section{B.4.7 Internal Codes}

The various internal codes in the RETRIEVE routine are listed below by hexadecimal digits.

AREAN

\begin{tabular}{|c|c|}
\hline $\mathrm{X}^{\prime} 00000000^{\prime}$ & $\begin{array}{l}\text { No records within range of } \mathrm{GT}, \mathrm{GE}, \mathrm{LT} \text {, } \\
\text { LE or FROM-TO relations }\end{array}$ \\
\hline$X^{\prime} F D F F F F F F^{\prime}$ & $\begin{array}{l}\text { Attribute of specified keyword does not } \\
\text { exist in the file }\end{array}$ \\
\hline$X^{\prime} F E F F F F F F^{\prime}$ & Error in range of FROM-TO relation \\
\hline$X^{\prime} F F F F F F F^{\prime}$ & $\begin{array}{l}\text { Value of specified keyword does not exist } \\
\text { in the file }\end{array}$ \\
\hline other & $\begin{array}{l}\text { Number of records in file containing } \\
\text { specified keyword }\end{array}$ \\
\hline
\end{tabular}

ERRCODE

$$
\begin{array}{ll}
X^{\prime} 04^{\prime} & \text { Part of directory unretrievable } \\
X^{\prime O C '} & \text { Hardware error }
\end{array}
$$

ISAMIND

$$
\begin{array}{ll}
X^{\prime} O O^{\prime} & \text { Code for DECODE to return only } n^{*} \\
X^{\prime} F^{\prime} & \text { Code for DECODE to return } n^{*} \text { and HOLA's }
\end{array}
$$


FNCODE (inunction code)

$$
\mathrm{X}^{\prime 22} \text { Code for retrieval }
$$

PRCODE (Print code indicating method of output)

$\begin{array}{ll}X^{\prime} 00^{\prime} & \text { Output on Low Speed Terminal (LST) } \\ X^{\prime} 02^{\prime} & \text { Output on high speed terminal } \\ X^{\prime} 04^{\prime} & \text { Output to program in core format } \\ X^{\prime} 80^{\prime} & \begin{array}{l}\text { No output of actual records. Only output } \\ \text { number of satisfactory records. }\end{array}\end{array}$

B.4.8 Flowchart and Supplementary Diagrams

Figures B.4.a - B.4.g contain the flowchart for the RETRIEVE routine. Figures $B .4 . h-B .4 . j$ contain supplementary diagrams. 


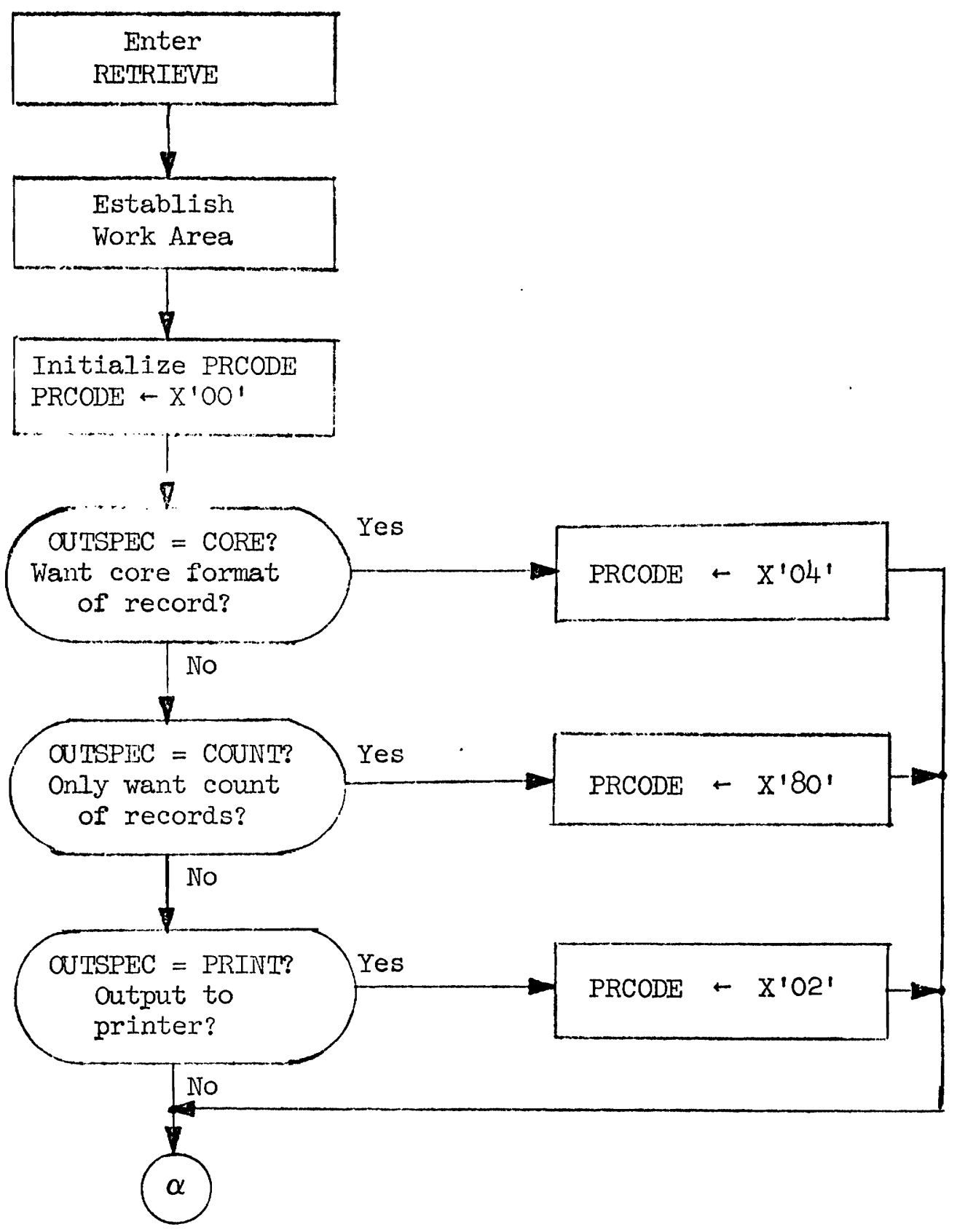

Figure B.4.a: RETRIEVE Initialization 


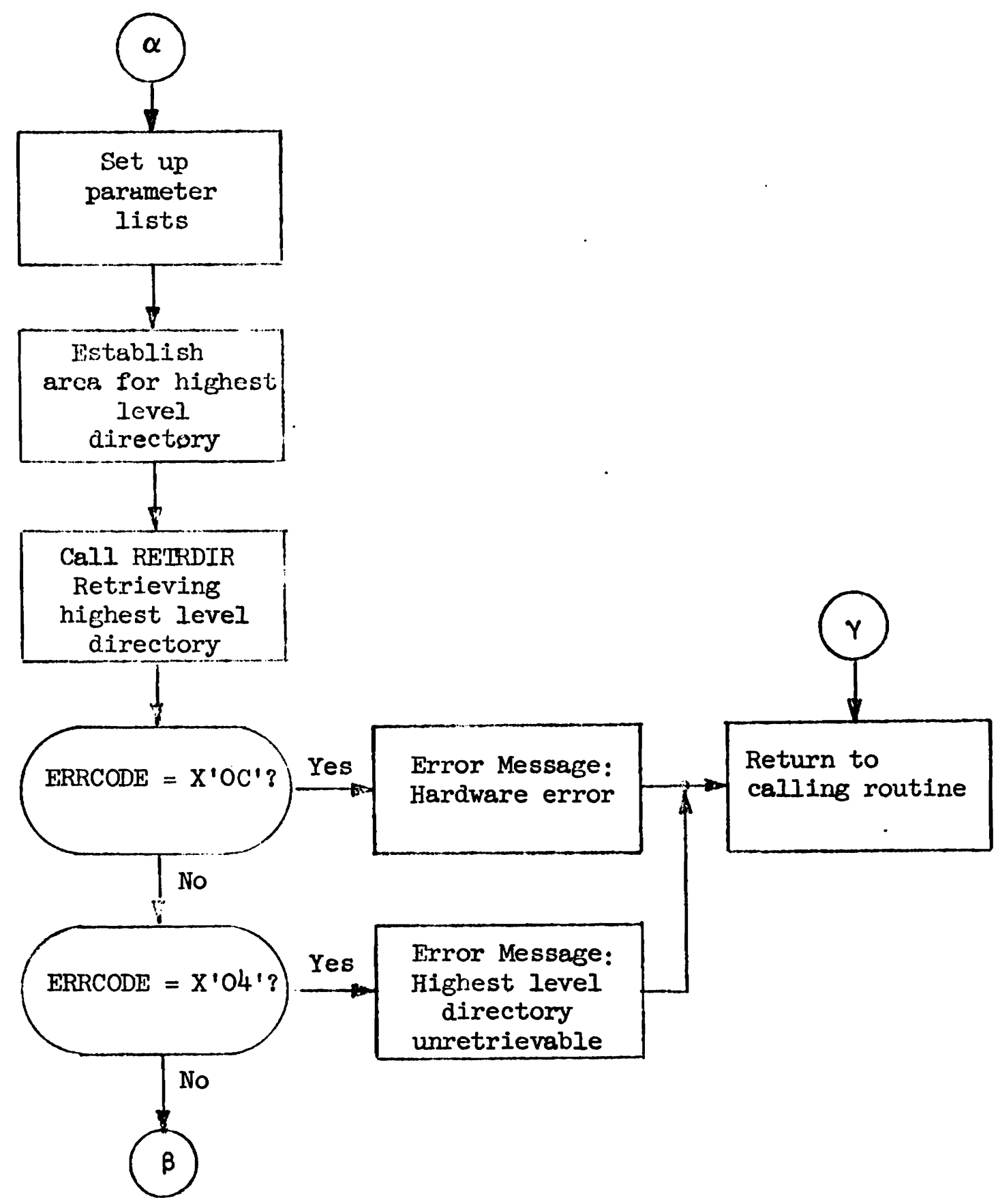

Figure B.4.b: Retrieve Highest Level Directory 


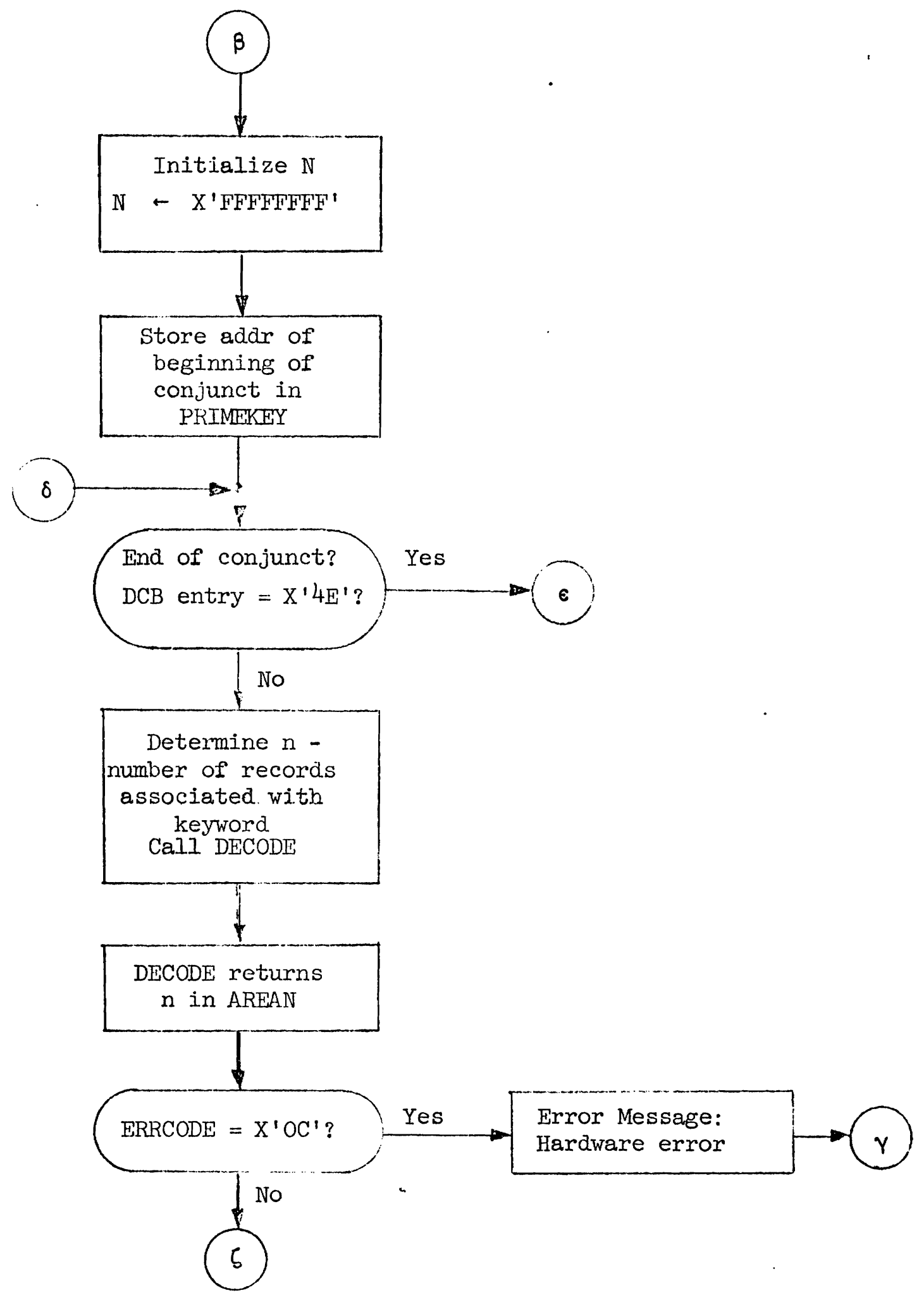

Figure B.4.c: Prime Keyword Selection 


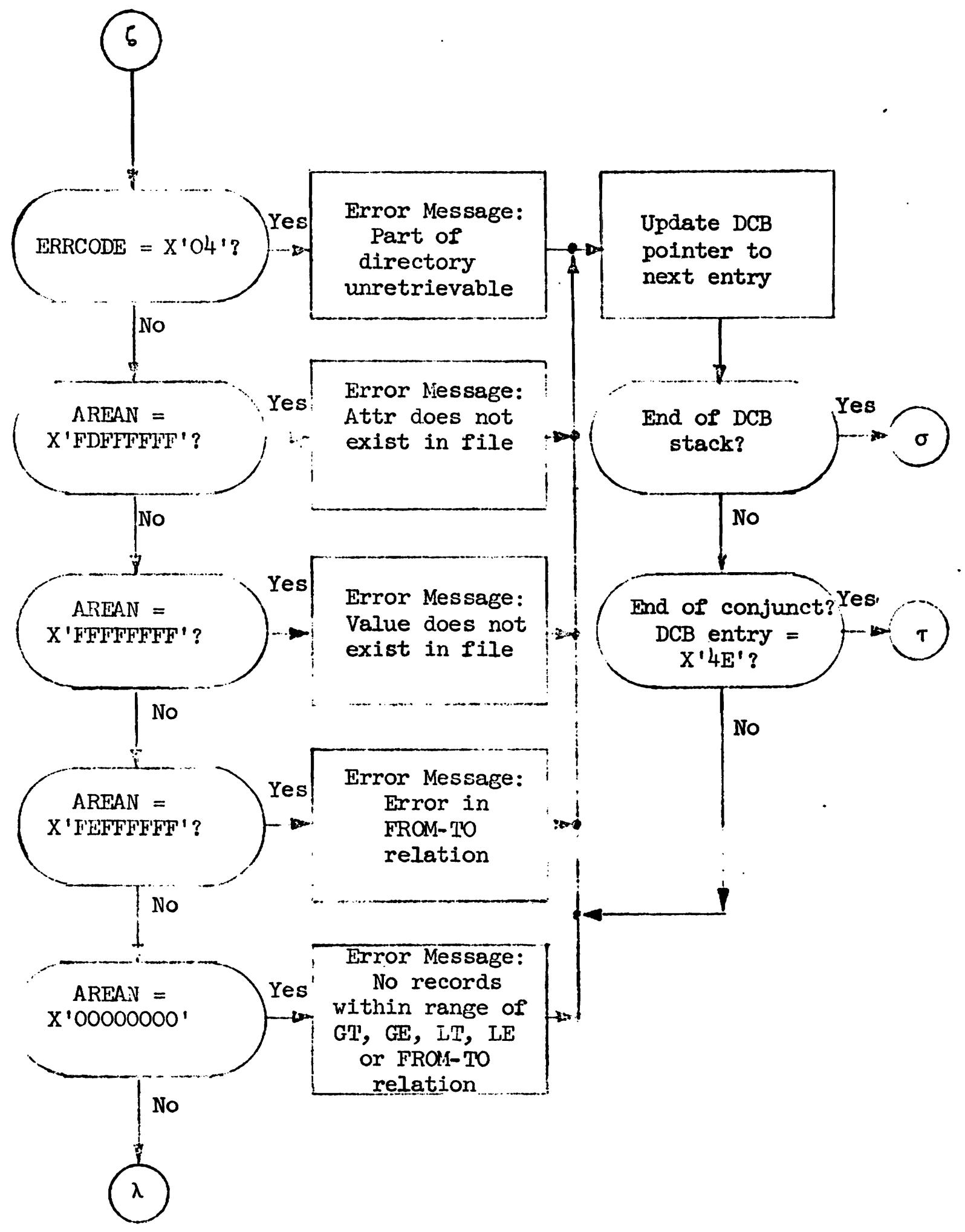

Figure B.4.d: Prime Keyword Selection 


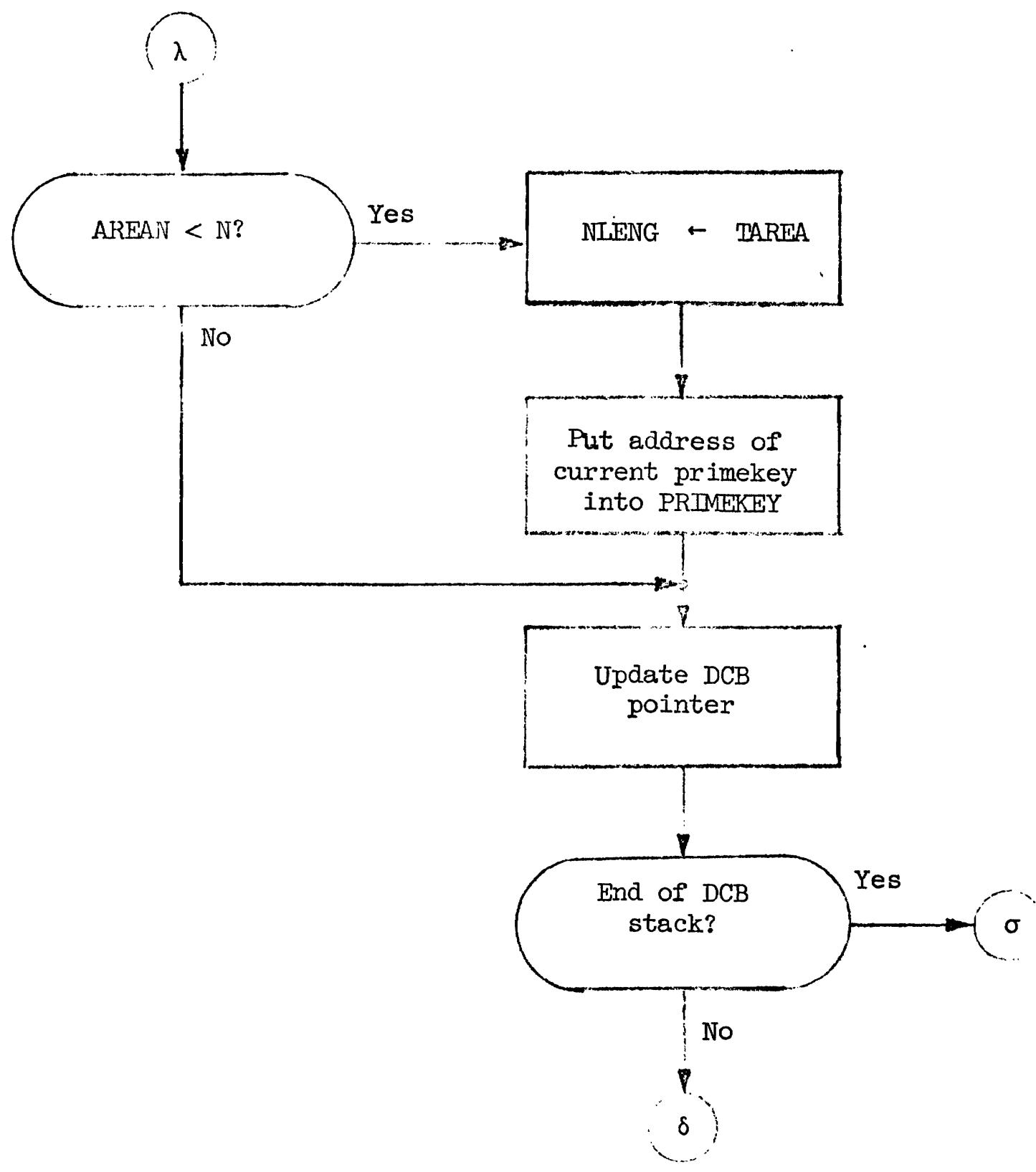

Figure B.4.e: Prime Keyword Selection 


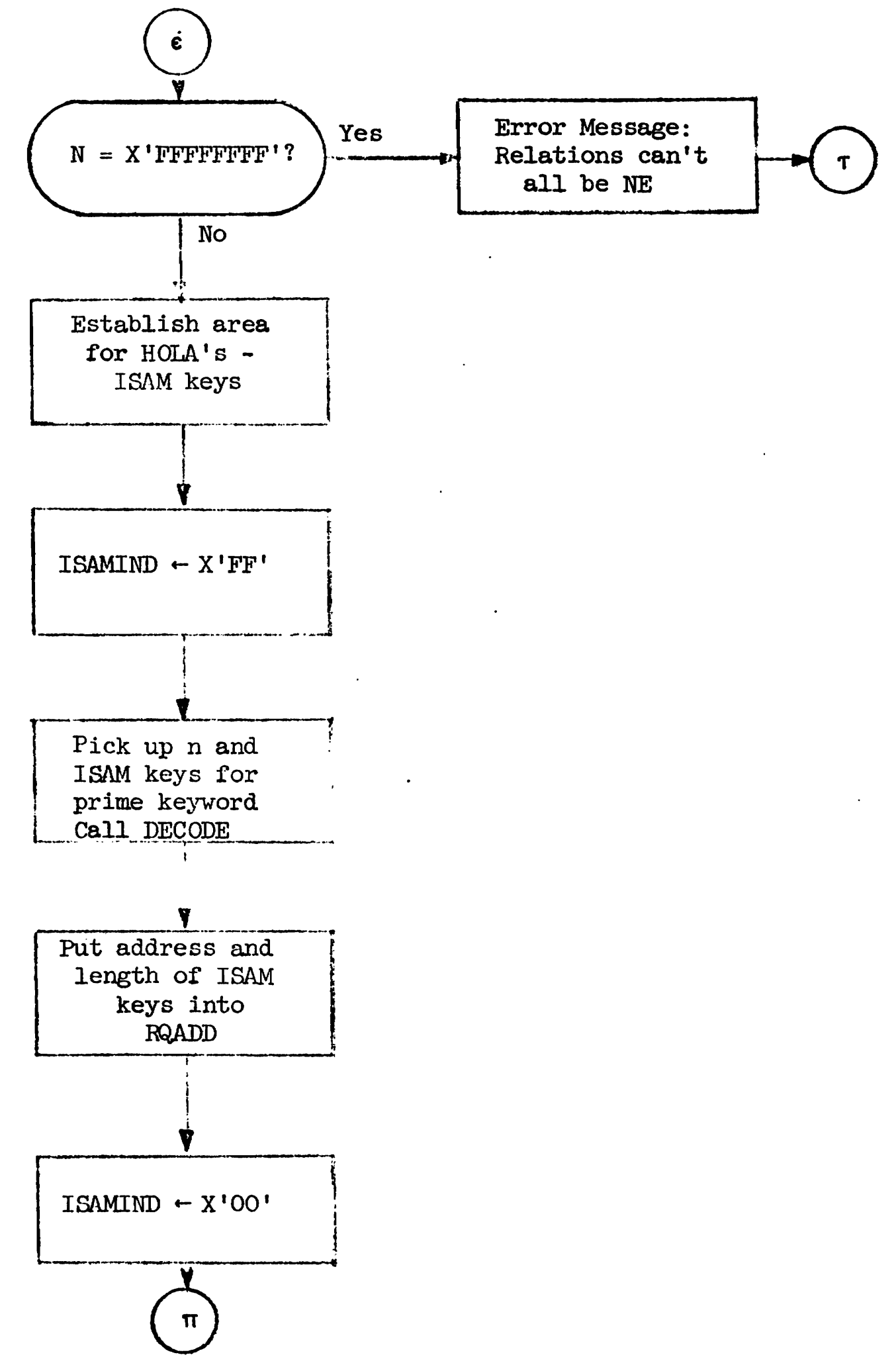

Figure B.4.f: Obtaining ISAM Keys 


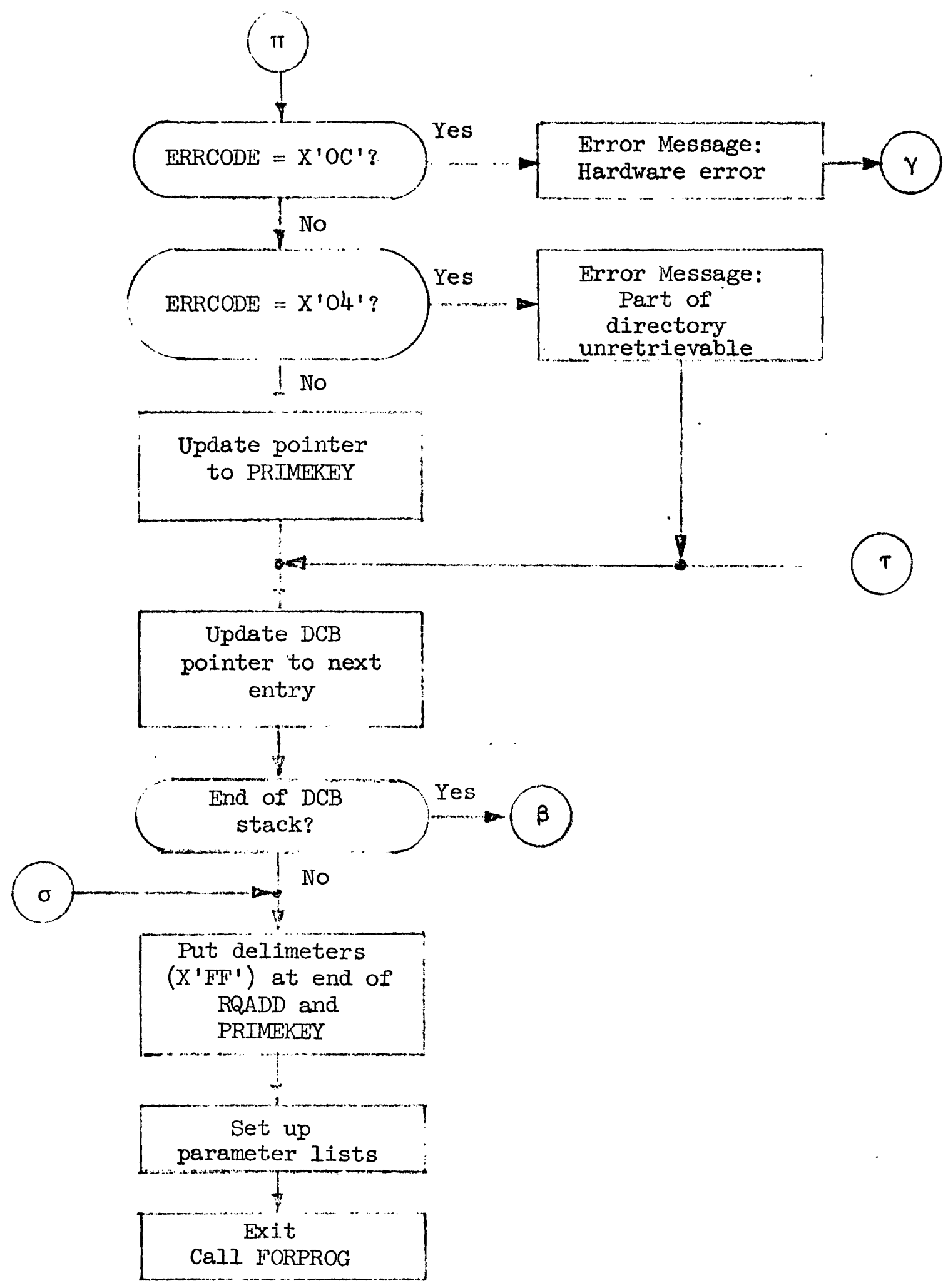

Figure B.4.g: Return to Process Next Conjunct and the Exit 
Key Information Buffer

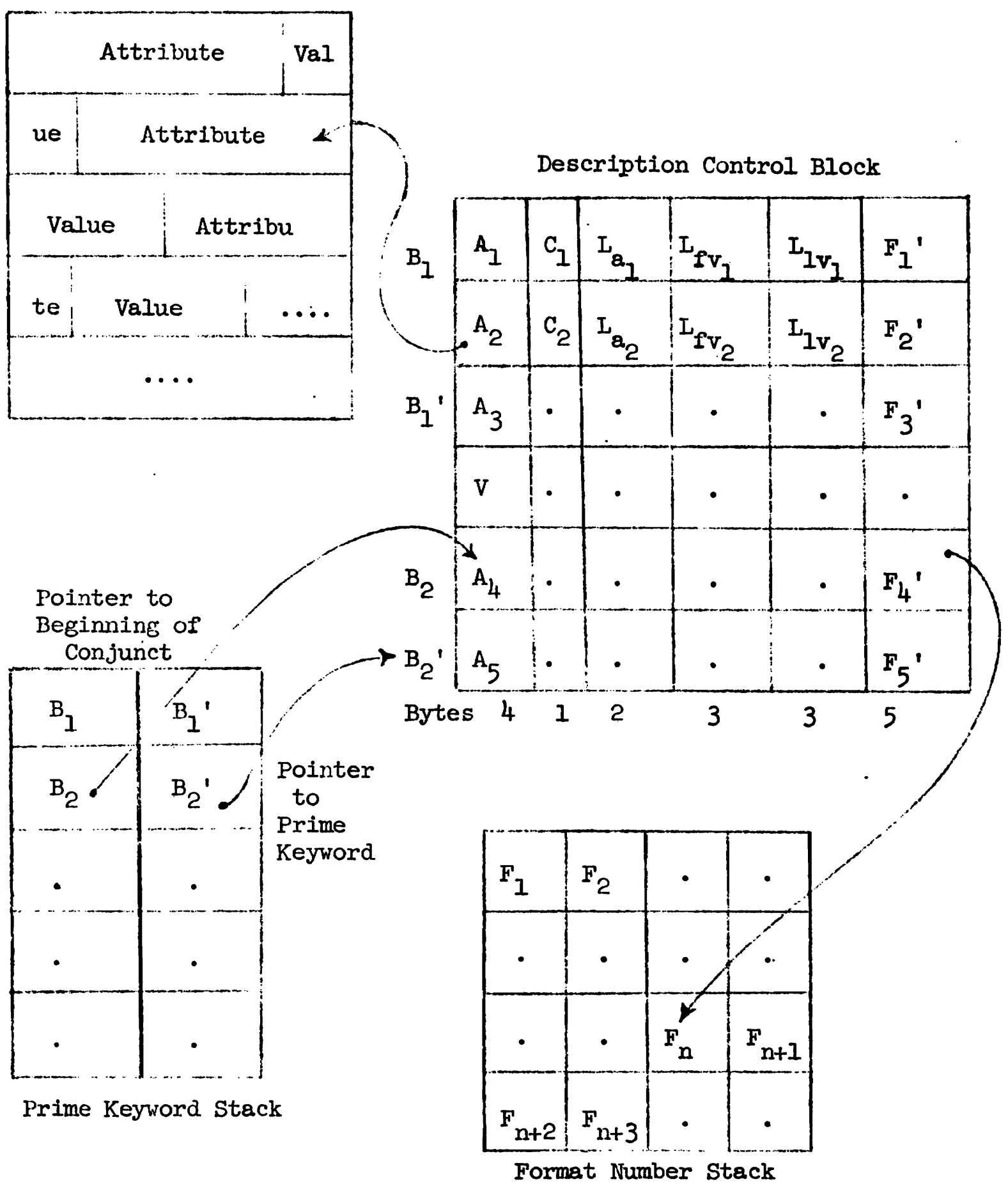

Figure B.4.h: Important Areas Used in the RETRIEVE and FORPROG Routines 
Description of Control Block Areas for Figure B.4.h •

$A_{i}$ is a pointer to the beginning of the $i^{\text {th }}$ keyword that is stored in the Key Information Buffer. The attribute and value(s) are stored in their entirety, i.e. exactly the way the user specified them.

$C_{i}$ is the control code that indicates the relation between the attribute and the value.

$I_{a_{i}} \quad$ is the length of the $i^{\text {th }}$ attribute.

$I_{f v_{i}}$ is the length of the first value of the $i^{\text {th }}$ keyword.

$\mathrm{I}_{I v_{i}}$ is the length of the last value of the $i^{\text {th }}$ keyword.

$\mathrm{F}_{i}^{\prime} \quad$ is the pointer to the beginning of a list of format numbers associated with the attribute.

Prime Keyword Stack Areas:

$B_{i}$ is the pointer to the beginning of the $i^{\text {th }}$ conjunct in the Description Control Block.

$B_{i}$ is the pointer to the prime keyword in the $i^{\text {th }}$ conjunct in the Description Control Block.

$F_{i}^{\prime}$ will appear as follows:

\begin{tabular}{|c|c|}
\hline $\begin{array}{c}\text { Address of beginning } \\
\text { of list }\end{array}$ & $\begin{array}{c}\text { \# of elements } \\
\text { in list }\end{array}$ \\
\hline
\end{tabular}

4 bytes 1 byte 


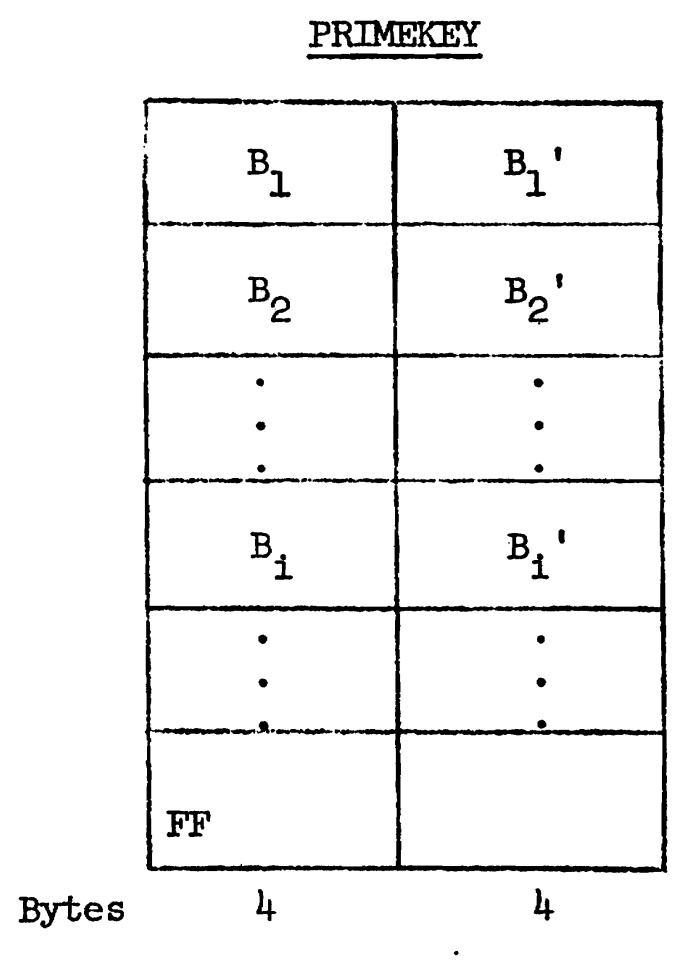

where $B_{i}$ : Pointer to beginning of conjunct in DCB $B_{i}^{\prime}$ : Pointer to prime keyword of conjunct beginning with $B_{i}$

Note: $X^{\prime} F F^{\prime}$ on a $B_{1}$ boundary indicates the end of the stack. 


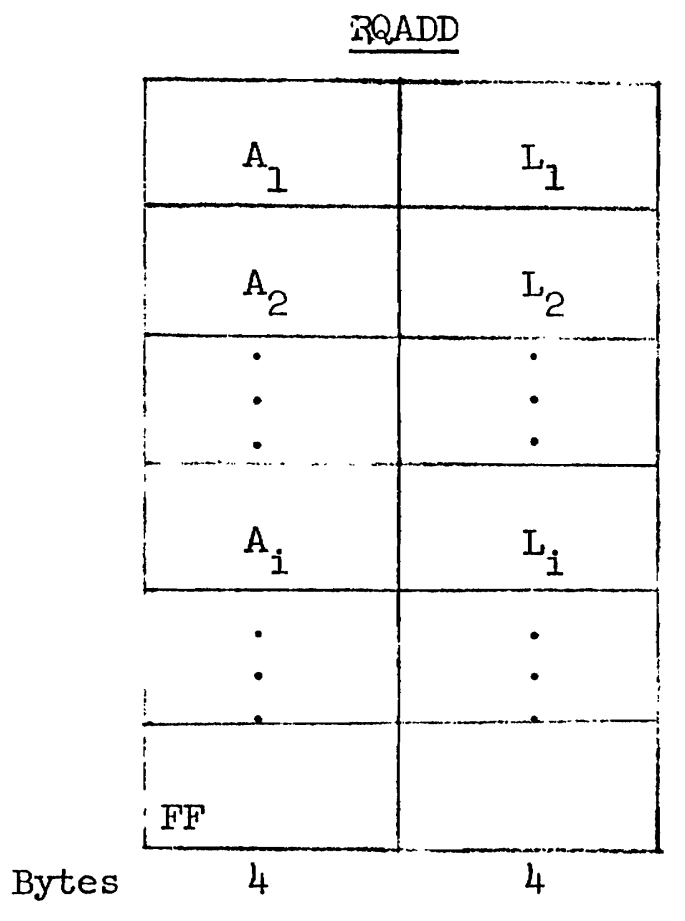

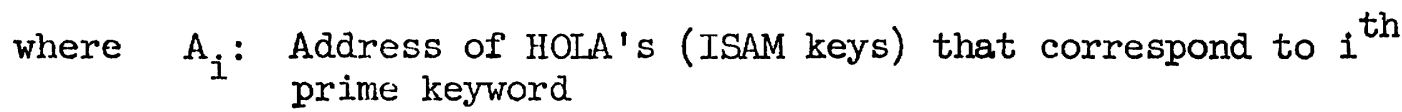

$I_{i}:$ Total length of ISAM keys

Note: $X^{\prime} F^{\prime}$ on an $A_{i}$ boundary indicates the end of the stack.

Figure B.4.j: RQADD Area 


\section{B.5 Routine FORPROG}

The FORPROG routine determines and lists the record format numbers for each attribute in the user's request description. The address of each Iist is placed in the 14th - 17th bytes of the DCB entry for the corresponding attribute. The number of associated format numbers is placed in the last byte of the DCB entry (see Figure B.4.h).

\section{B.5.1 Entry Points}

FORPROG is the only entry point in the routine.

\section{B.5.2 Exit Points}

FORPROG has three exit points. One is the normal exit point and the other two are used when an error occurs. The normal exit point begins at DONE where a call for the ESTAB entry of RETALG is issued [4]. The error exits are at FSPCENI and FSPCEN2.

\section{B.5.3 Input Parameter List}

The address of the input parameter list (PLFOR) must be in Register 1 and Register 13 must contain the address of the calling routine's save area.

\begin{tabular}{|c|c|c|}
\hline Name & Bytes & Content \\
\hline PLFOR & DSECT & \\
\hline PFCB & $0-3$ & Address of FCB \\
\hline PKIB & $4-7$ & Pointer to $\mathrm{KIB}$ \\
\hline INDAI & 8 & Code for Authority Item Checking routine \\
\hline PDPRKY & $8-11$ & $\begin{array}{l}\text { Pointer to PRIMEKEY Stack for Authority } \\
\text { Item Checking routine }\end{array}$ \\
\hline PPRMKY & $8-11$ & Pointer to PRTMEKKEY Stack \\
\hline RFBADD & $12-15$ & Address of RFB \\
\hline AIRQADD & $16-19$ & Pointer to RQADD \\
\hline PRECNO & $20-21$ & Number of requested records \\
\hline
\end{tabular}




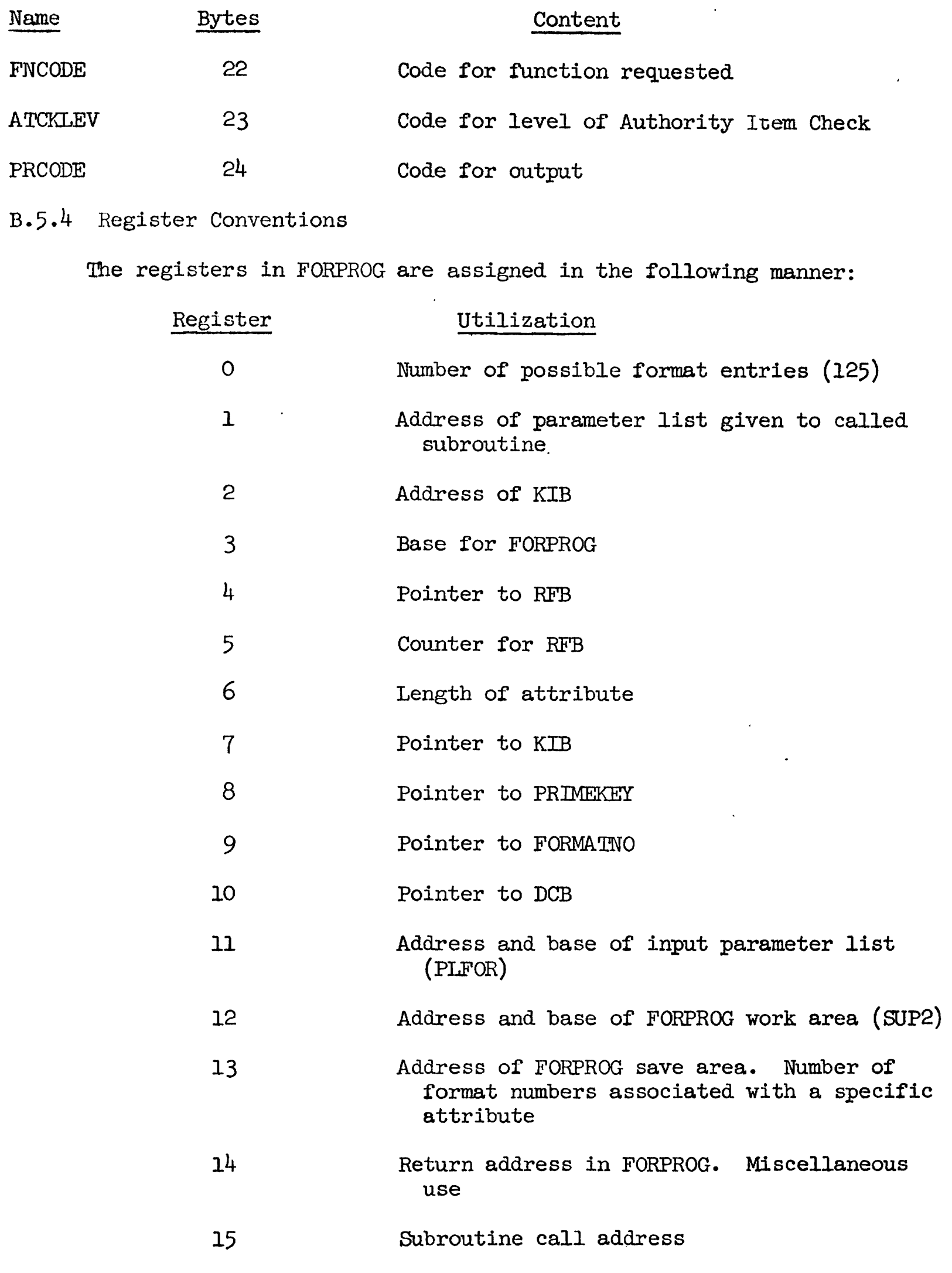


B.5.5 Internal Work Area

The internal work area used by FORPROG is called SUP2.

\begin{tabular}{lcl} 
Name & Bytes & \multicolumn{1}{c}{ Content } \\
SUP2 & DSECT & Temporary storage \\
ALFULI & $0-3$ & Current address in FORMATNO \\
PLFRST! & $4-7$ & Save area for FORPROG \\
SAVE & $8-79$ & Temporary storage \\
ALHAIJF & $80-81$ & Format number that is being checked \\
TFORNUM & $82-83$ & List of satisfactory format numbers \\
FORMATNO & $84-331$ & \\
B.5.6 Internal Codes &
\end{tabular}

INDAI

$$
\begin{aligned}
& \mathrm{X}^{\prime} \mathrm{FF}^{\prime} \quad \begin{array}{l}
\text { Indicates entrance is from the Authority } \\
\text { Item Checking routine }
\end{array}
\end{aligned}
$$

FNCODE (Function code)

$$
\mathrm{X}^{\prime 22} \quad \text { Code for retrieval }
$$

MARKER

X'FFF' Placed in the 6 th and 7 th bytes of the $\mathrm{DCB}$ entry to indicate a no attribute case

\section{B.5.7 Flowchart}

Figures B.5.a - B.5.d contain the flowchart for the FORPROG routine. 


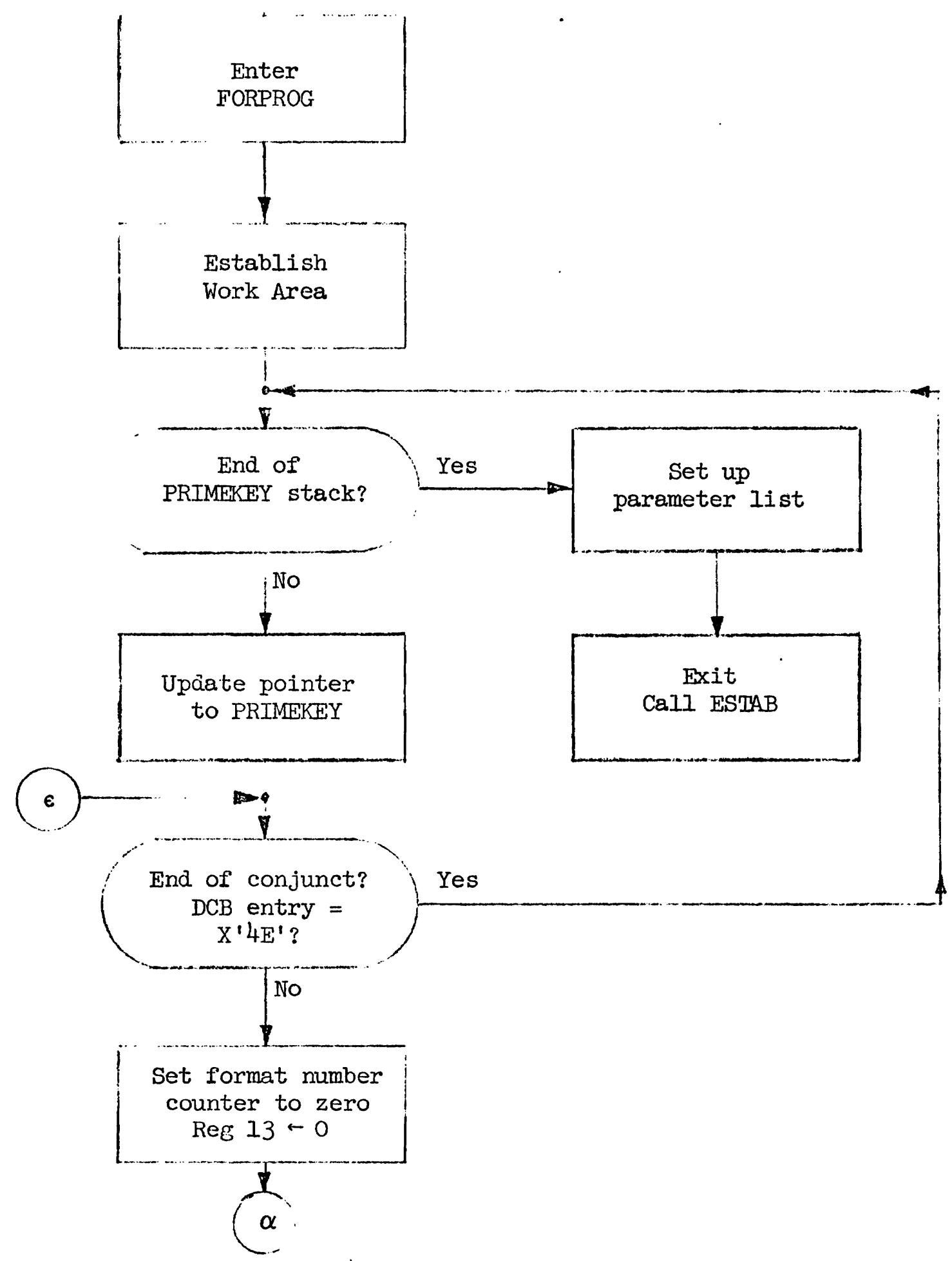

Figure B.5.a: FORPROG Initialization 


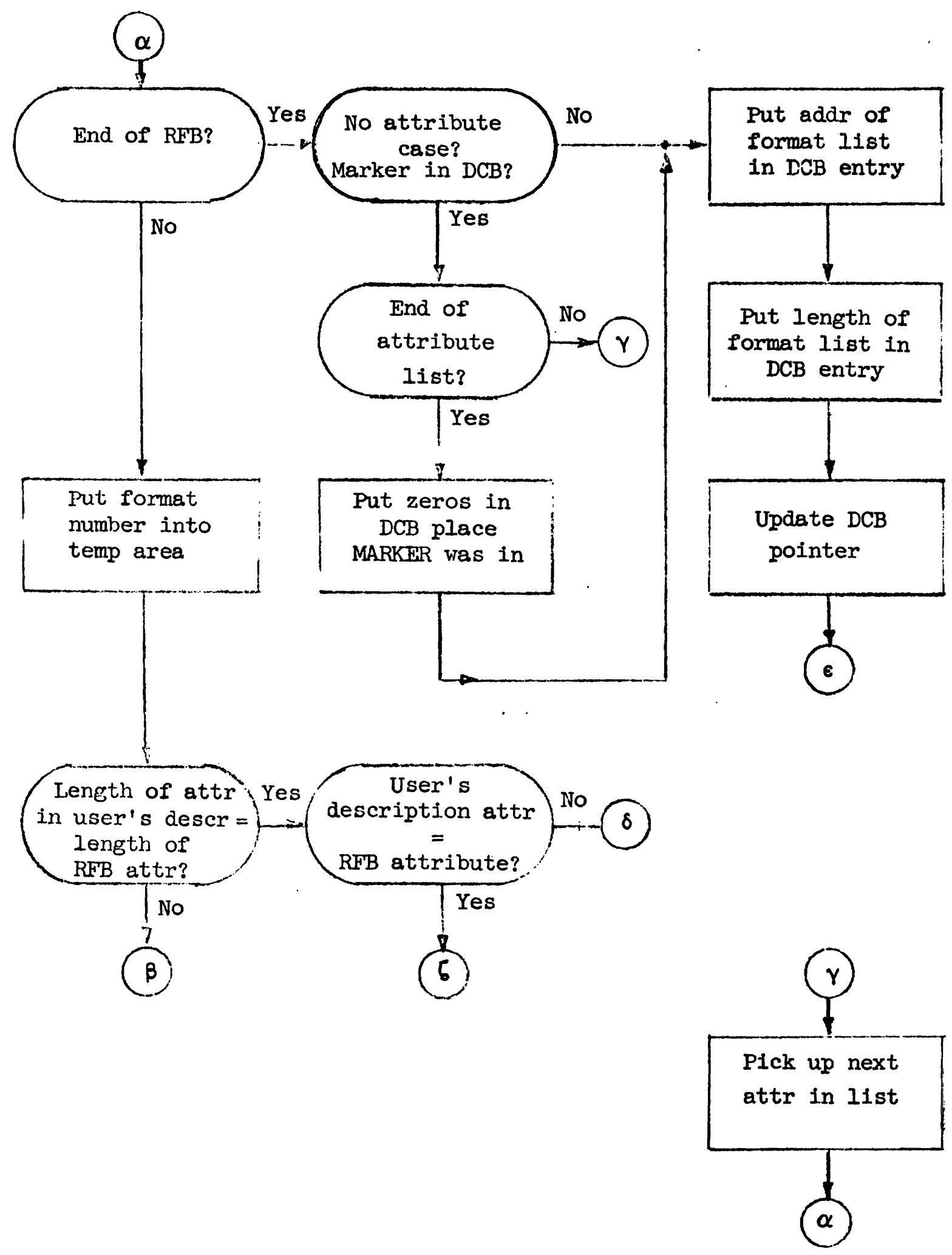

Figure B.5.b: Obtaining Format Numbers 


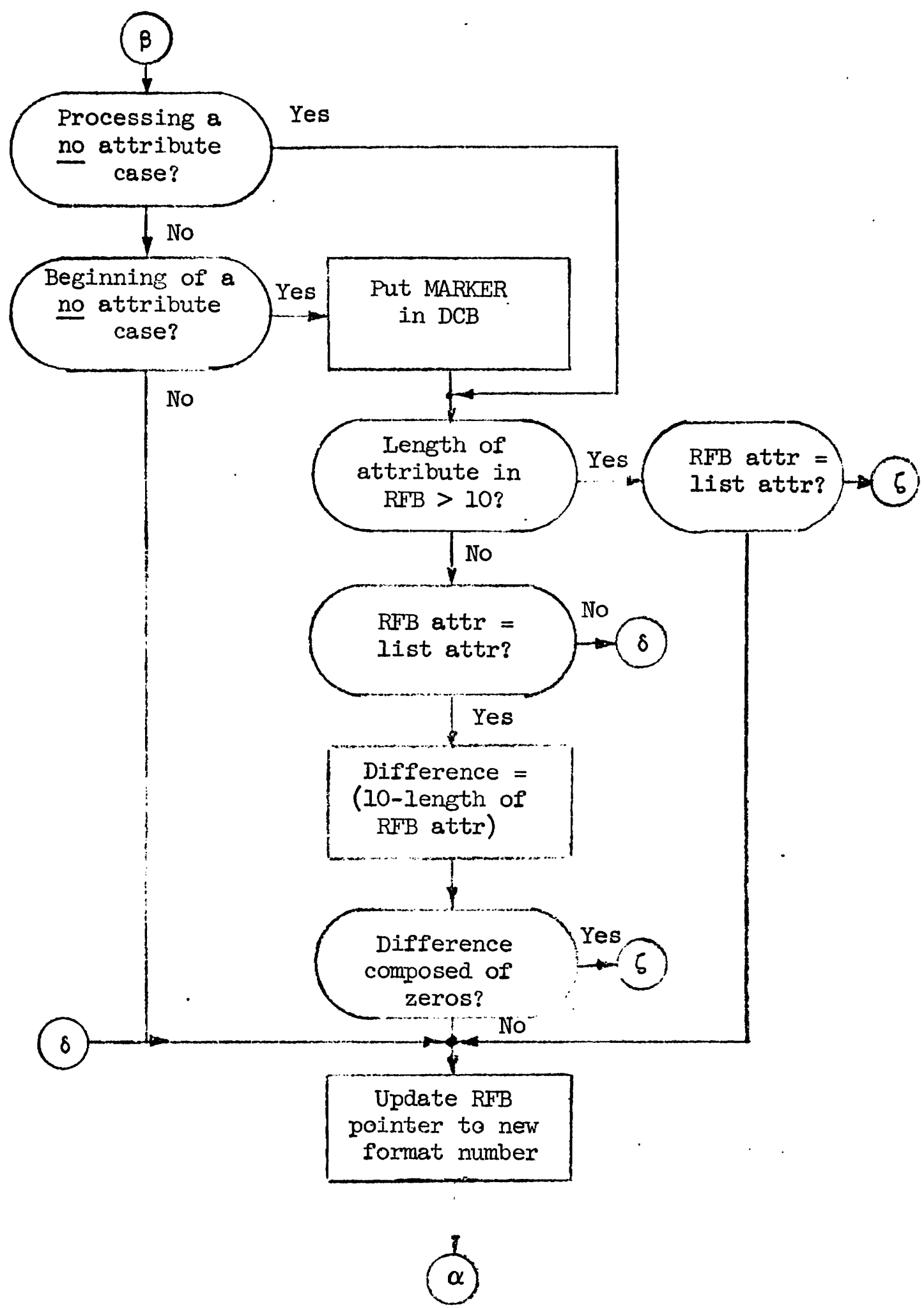

Figure B.5.c: Processing No Attribute Case 


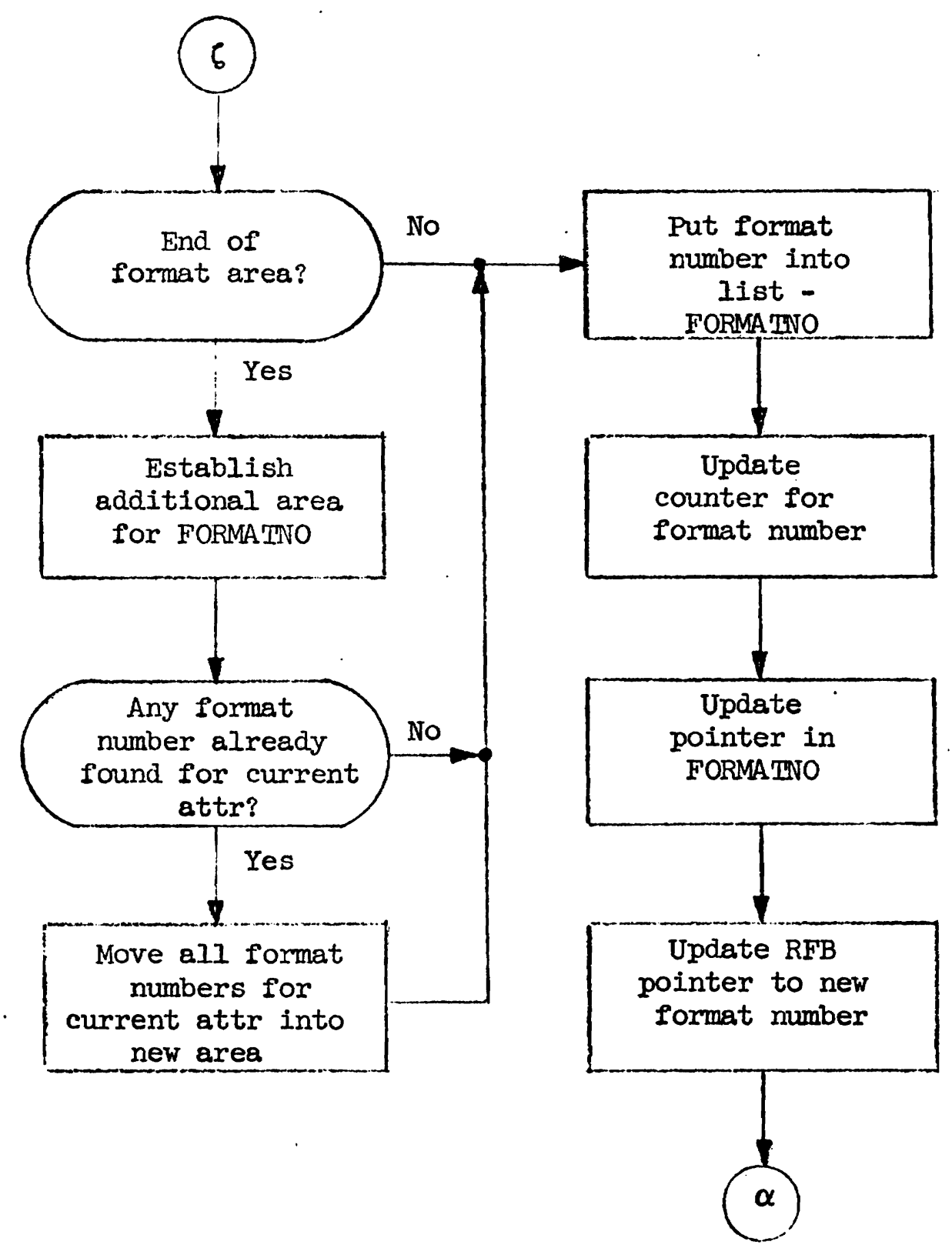

Figure B.5.d: Establishing Additional Area for Format Numbers 


\section{B.6 Routine CI.ISEPROC}

The CLSEPROC routine is the one that implements the Close Function of the EDMF. It indicates in the SSB chain that the specifita partition(s) have been closed and also removes the corresponding FSB entries.

\section{B.6.1 Entry Points}

CLSEPROC has two entry points. CLSEPROC is the SVC entrance while the command entrance is at COMDCLSE.

\section{B.6.2 Exit Points}

There is only one exit point for this routine. It begins at CKEXIT where control is returned to the calling program.

\section{B.6.3 External Subroutine Calls}

Three external subroutines are called by CLSEPROC. The first is to the location ESQCAT to obtain the task number. The second is to the entry point SSBACQR of the SSBOPIR routine [9]. This is used to obtain the SSB chain for the specified user. The third external subroutine that is called upon is FSBOPTR [9]. It is through the use of the FSBOPTR routine that FSB entries are removed. The DSECT's that are associated with the SSBOPTR and the FSBOPTR routines are the following:

\begin{tabular}{lll} 
Name & Bytes & \multicolumn{1}{c}{ Content } \\
SSB & DSECT & SSB Header \\
SSBHDR & $0-7$ & Address of User's Authority Item \\
SSBUAI & $0-3$ & Address of FCB for File Information File \\
SSBFIF & $4-7$ & SSB text \\
SSBTXT & $8-91$ & 2 bytes - length of file name \\
SSBFNAM & $8-63$ & 54 bytes - file name \\
SSBCL & $64-67$ & Control Information \\
& 64 & Type of request
\end{tabular}




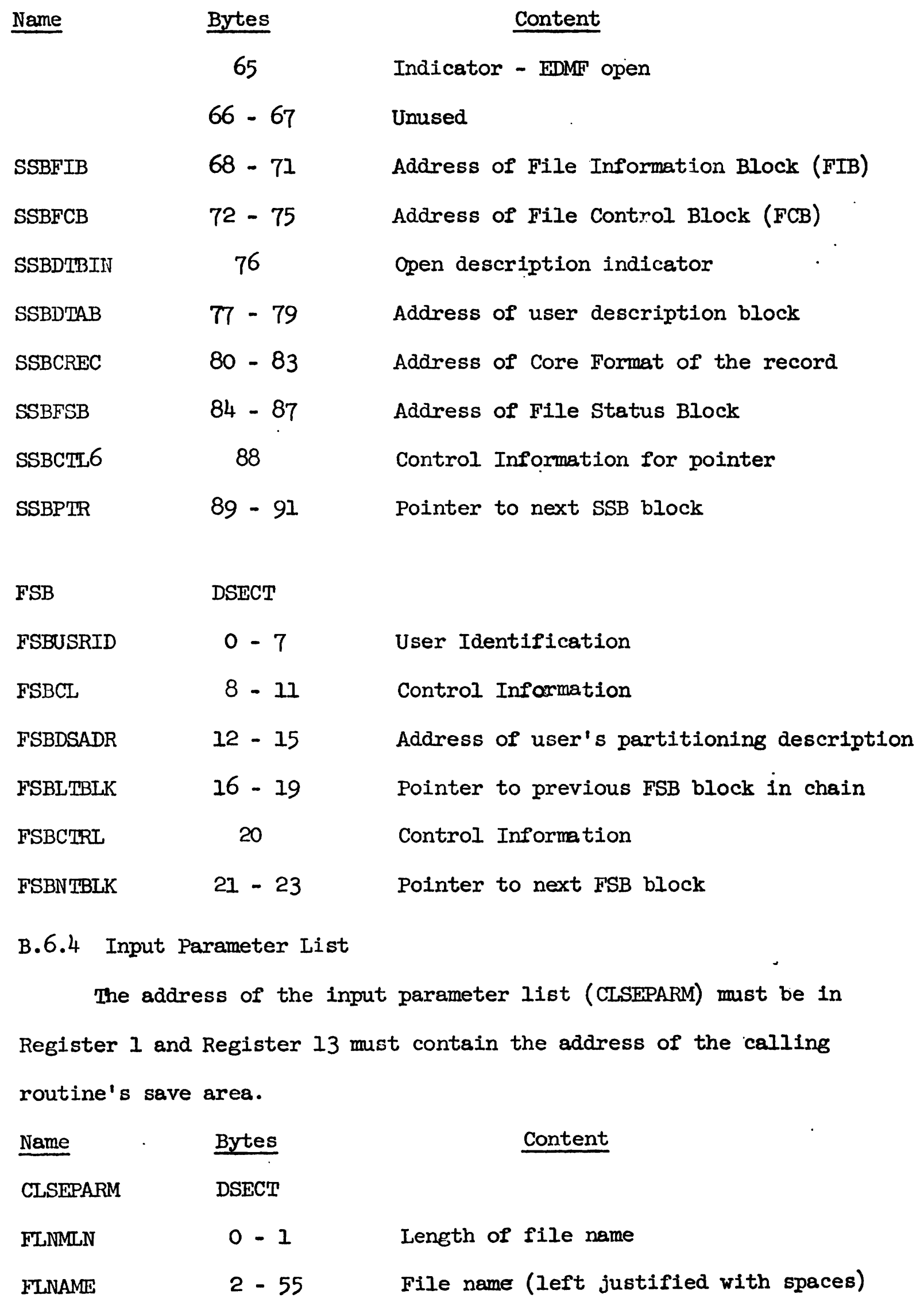




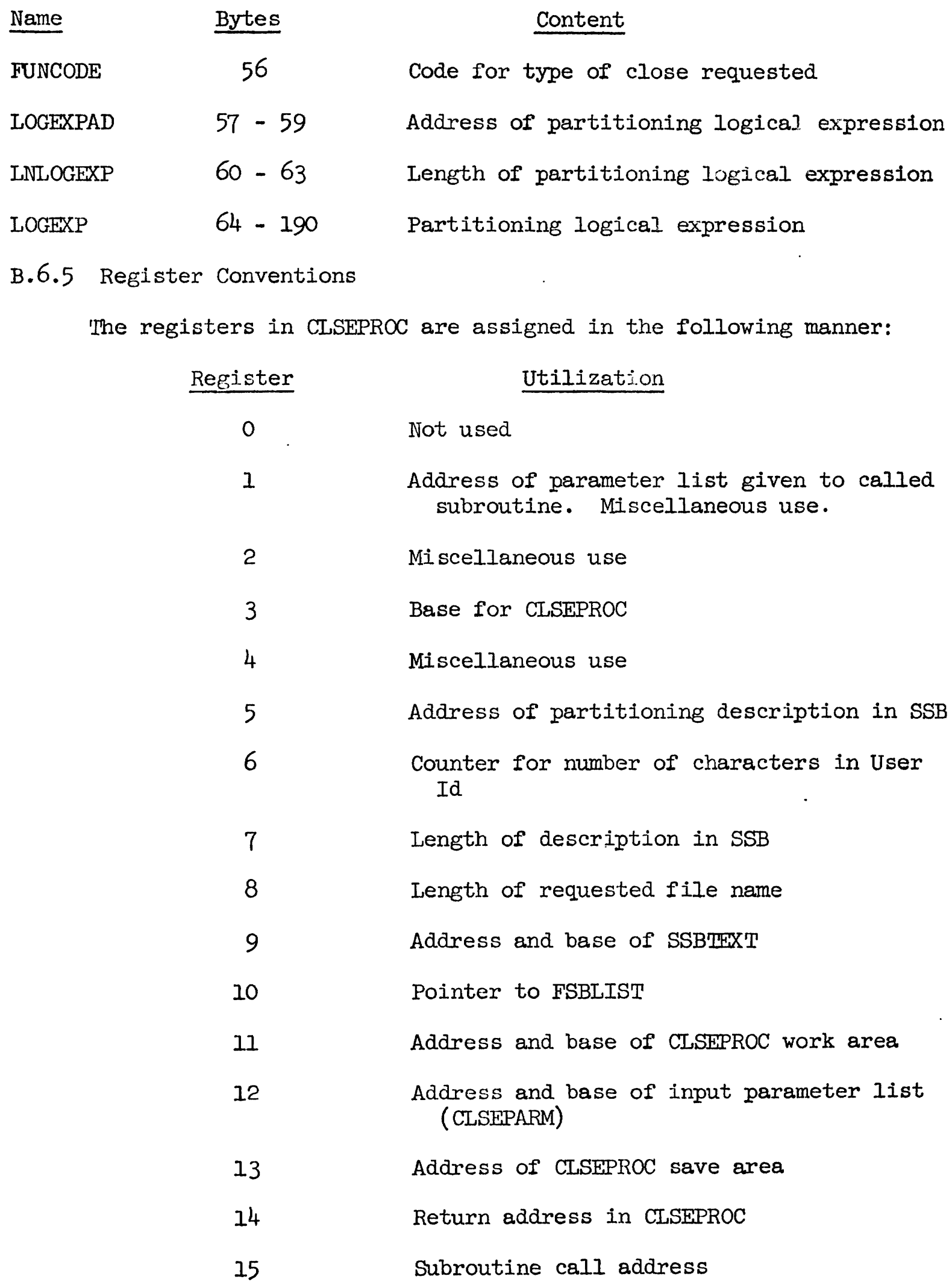


B.6.6 Internal Work Area

CLSEWORK is the internal work area used by the CLSEPROC routine. It contains the parameter list (FSBLIST) that is passed to the FSBOPIR routine. The work area has the following format:

Name

Bytes

Content

CLSEWORK

DSECT

DPRM2

$0-7$

Parameter area for error messages

CLSAVE

$8-79$

Save area for CLSEPROC

ATMODEAR

$80-83$

Address of area for TMODE macro

$84-85$

Length of area for TMODE macro

TMODEAR

$86-115$

Area for TMODE macro

USERID

$116-123$

User Identification

FSBLIST

$124-207$

List of addresses of FTSB blocks to be removed

ADRSIPACK

$208-211$

Address of stack area of SVC

TEMPA

$212-262$

Temporary area

TSKNUM

263

Task number

SWI

264

Code - found appropriate SSB block

CHKCODE

265

Code for errors

DNTMOPN2

$266-26 \overline{7}$

$268-270$

DMESSI

$271-366$

For re-entrant error message

SW2

$$
367
$$

Code for macro entrance

B.6.7 Internal Codes

The various internal codes in the CLSEPROC routine are listed below by hexadecimal digits.

- $\mathrm{CHKCODE}$

$$
\mathrm{X}^{\prime} 04^{\prime} \quad \text { SSB exists and has been acquired }
$$


FSBCTRL

$X^{\prime} \mathrm{FF}^{\prime}$

FUNCODE (Function code)

$\begin{array}{ll}X^{\prime} 48^{\prime} & \text { Close all partitions } \\ X^{\prime} 49^{\prime} & \text { Close specified partition }\end{array}$

SSBCI+1

$\begin{array}{ll}X^{\prime} O O^{\prime} & \text { File partition EDMF closed } \\ X^{\prime} F^{\prime} & \text { File partition EDMF open }\end{array}$

SSBCTH 6

$$
X^{\prime} F^{\prime}{ }^{\prime}
$$

$X^{\prime} F^{\prime}{ }^{\prime}$

SSBDTBIN

$$
X^{\prime} F^{\prime} F^{\prime}
$$

SWI

$$
X^{\prime} F F^{\prime}
$$
block
Code that indicates good pointer in FSB block

Code that indicates good pointer in SSB
Code that indicates user description block present

SW2

$$
\mathrm{X}^{\prime} \mathrm{FF}^{\prime} \quad \text { Entrance from macro }
$$

B.6.8 Return Codes

All return codes can be found in the right-most byte of Register 15 and they are listed below by hexadecimal digits.

$$
\begin{array}{ll}
X^{\prime} 00^{\prime} & \text { Everything } 0 . K . \\
X^{\prime} 04^{\prime} & \text { Appropriate SSB block does not exist }
\end{array}
$$

B.6.9 Flowchart and Supplementary Diagram

Figures B.6.a - B.6.C contain the flowchart for the CLSEPROC routine while Figure B.6.d contains a supplementary diagram. 


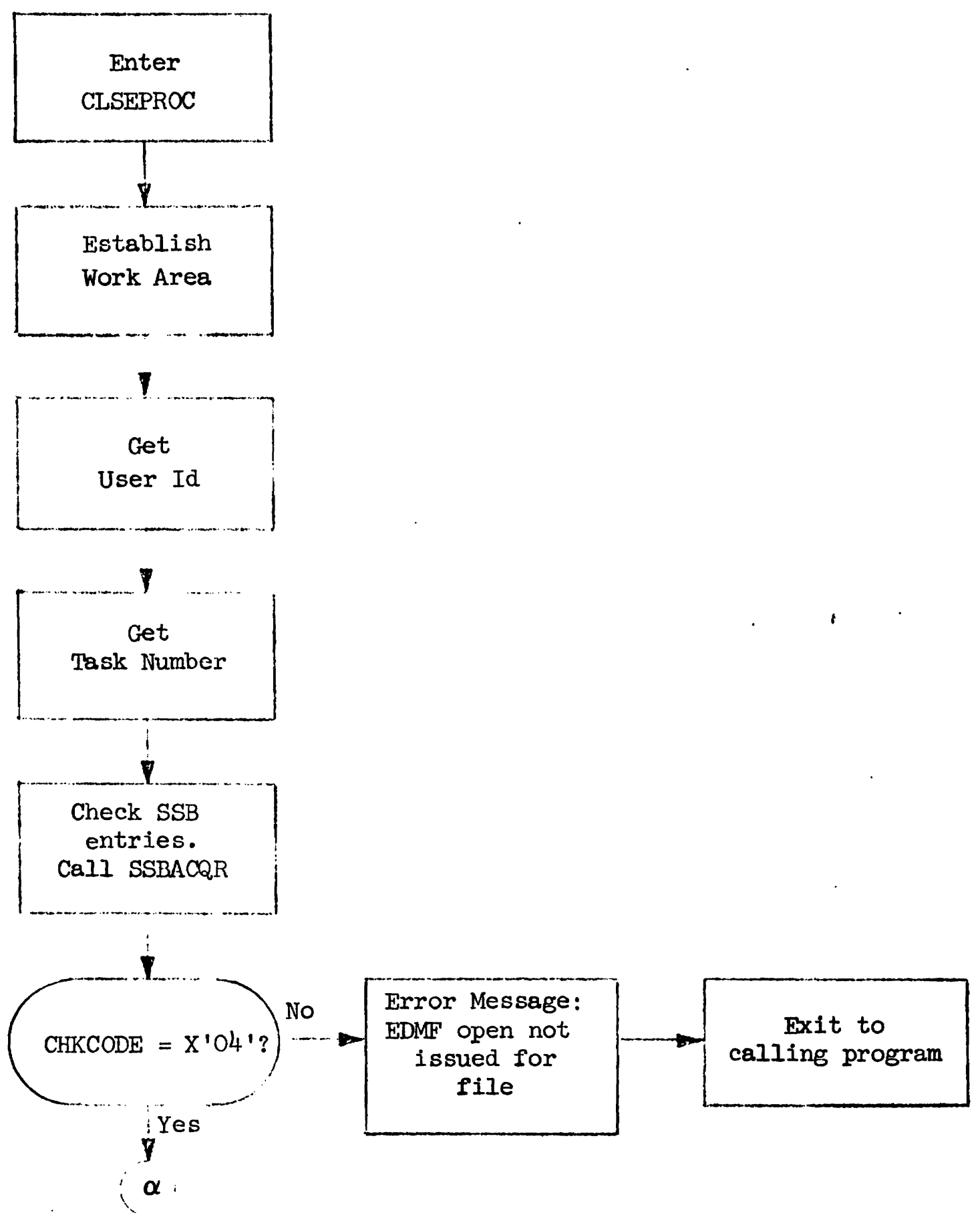

Figure B.6.8: CLSEPROC Initialization 


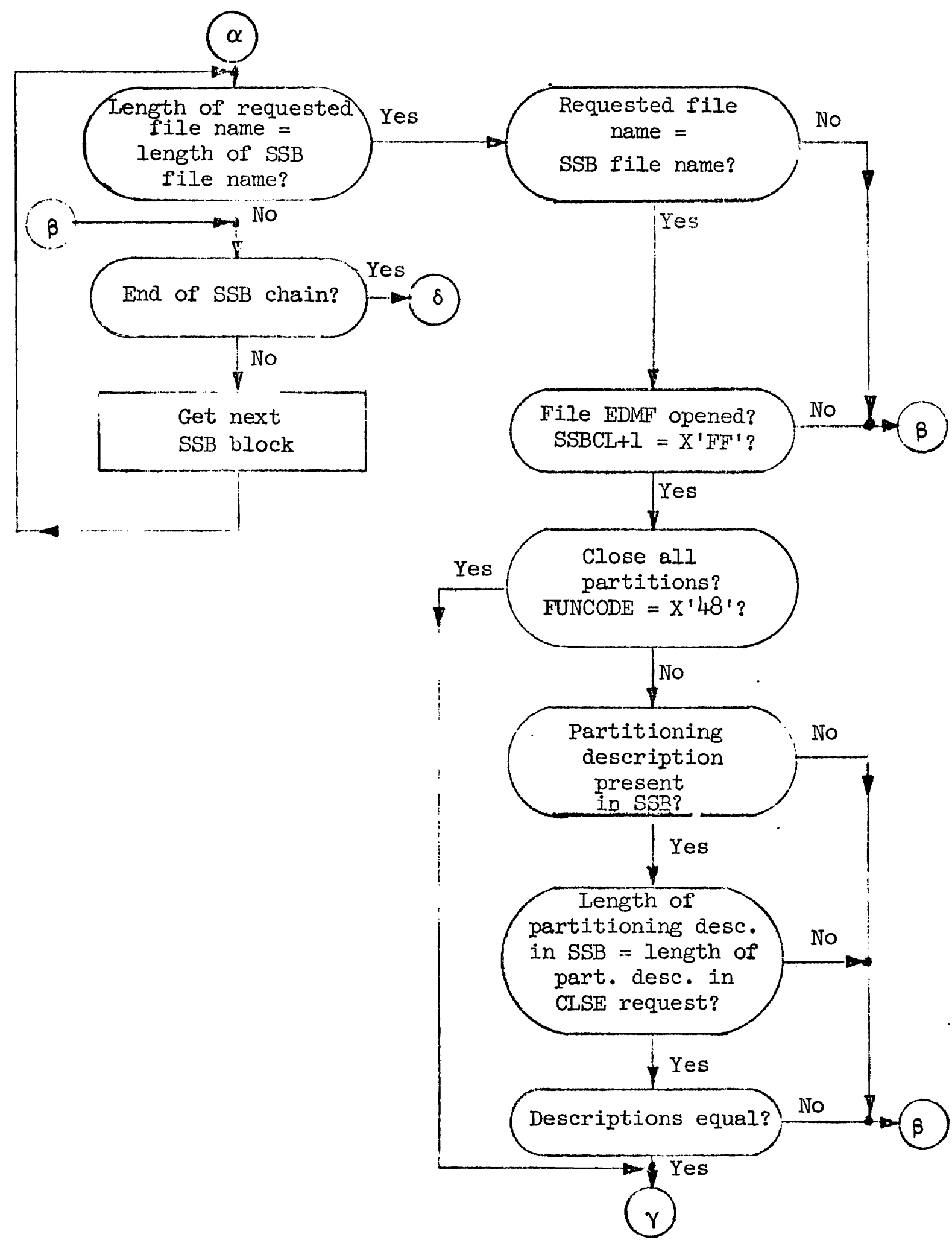

Figure B.6.b: SSB Check 


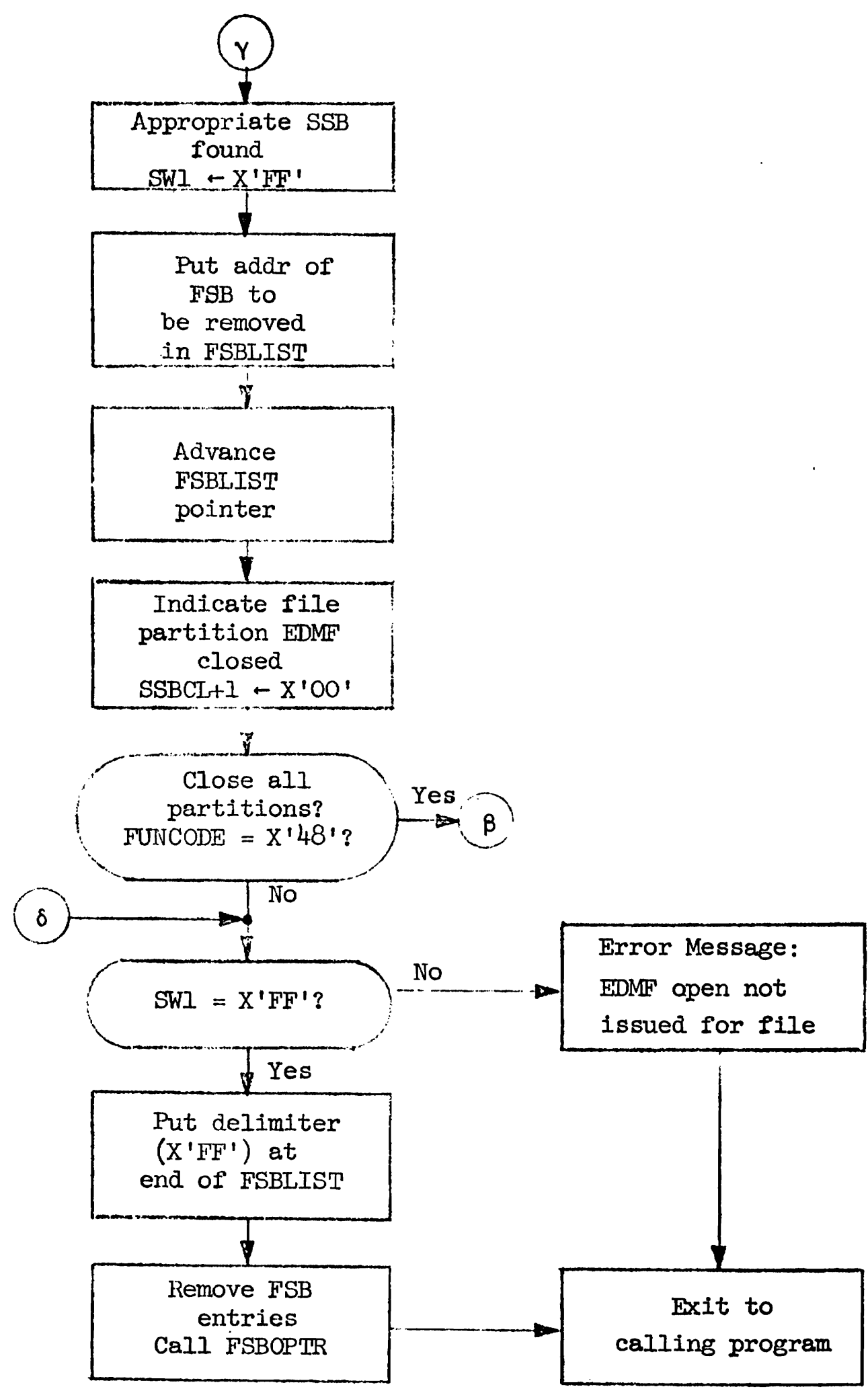

Figure B.6.c: Closing SSB, Setting Up FSBLIST and Exit 


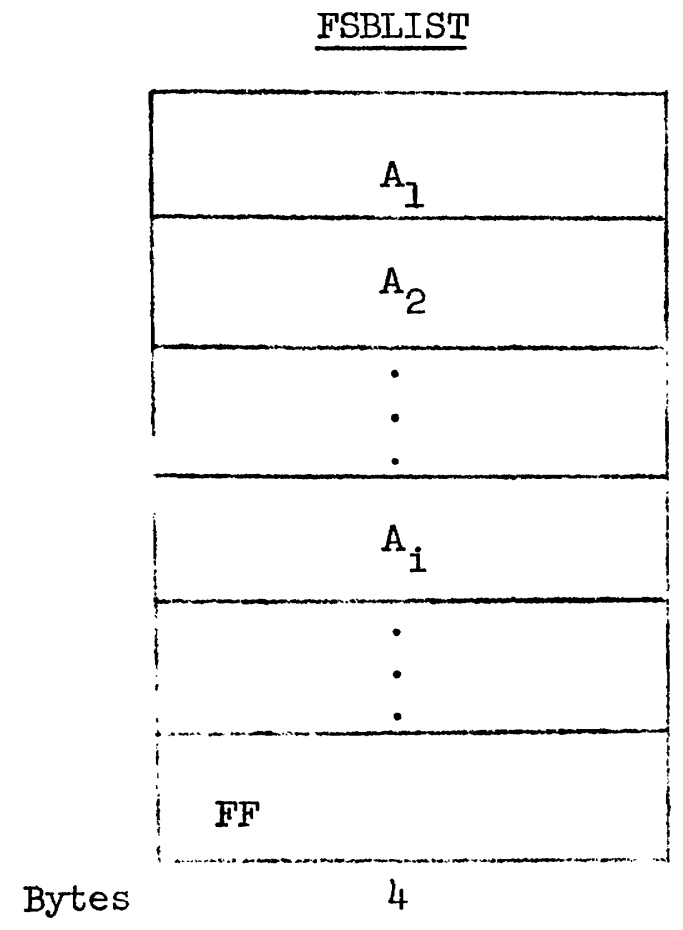

where $A_{i}$ : Pointer to a File Status Block

Note: $X^{\prime} F F^{\prime}$ on a boundary indicates the end of the stack.

Figure B.6.d 


\section{APPENDIX C}

CONTROL BLOCKS

C.I File Status Block

\begin{tabular}{|c|c|}
\hline $\begin{array}{l}8 \text { bytes } \\
4 \text { bytes } \\
4 \text { bytes } \\
4 \text { bytes } \\
4 \text { bytes }\end{array}$ & $\begin{array}{l}\text { User Identification } \\
\text { Control Information } \\
\text { Control--Address of user description block } \\
\text { Control--Pointer to previous FSB block } \\
\text { Control--Pointer to next FSB block } \\
\text { o0- null pointer }\end{array}$ \\
\hline
\end{tabular}

Notes on the File Status Block

1. Unless stated explicitly, all control information is 1 byte, all addresses are 3 bytes. 


\begin{tabular}{|c|c|}
\hline $\begin{array}{l}4 \text { bytes } \\
4 \text { bytes }\end{array}$ & $\begin{array}{l}\text { Control--Address of User's Authority } \\
\text { Item } \\
\text { Control--Address of FCB for FIF }\end{array}$ \\
\hline $\begin{array}{r}2 \text { bytes } \\
54 \text { bytes } \\
4 \text { bytes } \\
4 \text { bytes } \\
4 \text { bytes } \\
4 \text { bytes } \\
4 \text { bytes } \\
4 \text { bytes } \\
4 \text { bytes }\end{array}$ & $\begin{array}{l}\text { Length of Filename } \\
\text { Filename } \\
\text { Control Information } \\
\text { Control--Address of FIB for filename } \\
\text { Control--Address of FCB for filename } \\
\text { Control--Address of user description } \\
\text { block } \\
\text { Control--Address of core format record } \\
\text { Control--Address of corresponding FSB } \\
\text { block } \\
\text { Control--Pointer to next SSB entry } \\
\text { OO--null pointer } \quad \text { FF--good pointer }\end{array}$ \\
\hline
\end{tabular}

Notes on the Service Status Block

1. Unless stated explicitly, all control information is $I$ byte, all addresses are 3 bytes.

2. The header appears on the first SSB block only--all subsequent SSB entries contain only the text.

Ist $\mathrm{SSB}$ block $=8+84$ bytes $=92$ bytes

all subsequent $\mathrm{SSB}$ blocks $=84$ bytes

\section{C.2.I User Description Block}

4 bytes

$n$ bytes
Length of partitioning description Partitioning description 
C. 3 Record Format Block (RFB)

\begin{tabular}{|c|c|}
\hline $\begin{array}{l}4 \text { bytes } \\
2 \text { bytes } \\
2 \text { bytes }\end{array}$ & $\begin{array}{l}\text { Control Information } \\
\text { Pointer to first format } \\
\text { relative to first byte of RFB } \\
\text { Last format number assigned }\end{array}$ \\
\hline $\begin{array}{l}2 \text { bytes } \\
2 \text { bytes } \\
2 \text { bytes }\end{array}$ & $\begin{array}{l}\text { Format number } \\
\text { Control information } \\
\text { Relative address of first format }\end{array}$ \\
\hline $\begin{array}{l}2 \text { bytes } \\
2 \text { bytes } \\
2 \text { bytes }\end{array}$ & $\begin{array}{l}\text { Format number } \\
\text { Control information } \\
\text { Relative address of second format }\end{array}$ \\
\hline $\begin{array}{l}2 \text { bytes } \\
4 \text { bytes } \\
2 \text { bytes } \\
2 \text { bytes } \\
3 \text { bytes } \\
1 \text { byte } \\
2 \text { bytes } \\
4 \text { bytes } \\
2 \text { bytes } \\
n \text { bytes }\end{array}$ & $\begin{array}{l}\text { Format number } \\
\text { Type of format } \\
\text { Level number } \\
\text { Repetition number } \\
\text { Size of value } \\
\text { Control information } \\
\text { blank } \\
\text { Field protection data } \\
\text { Length of attribute } \\
\text { Full attribute name }\end{array}$ \\
\hline & $\cdot \cdot$ \\
\hline
\end{tabular}

HEADER

TABIE OF

CONTENTS

Notes on the Record Format Block

1. All relative addresses in the Table of Contents are relative to the first byte in the first format, hence a pointer to the first format is placed in the header. This arrangement obviates the need for changing relative addresses in the Table of Contents if new formats are added to the block.

2. Format numbers appear in the Table of Contents in order of their appearance in file records.

3. The Type of Format field may be used to indicate a program which processes the format. 
4. Like the size of value entry, the repetition number will not appear in the format if the format may repeat a variable number of times. Variable repetition is indicated by a bit in the control information.

5. Control information in the format entry is one byte long with the following specification:

abcd ee00

a: 0 Repetition number is variable

1 Repetition number is fixed

b: 0 Value size is variable

1 Value size is fixed

c: 0 Attribute is not in the directory

I Attribute is in the directory

d: 0 Attribute optionally appears in a record

1 Attribute appears in every record

ee: 00 Value is packed decimal

10 Value is alphabetic

01 Unassigned

11 Unassigned 